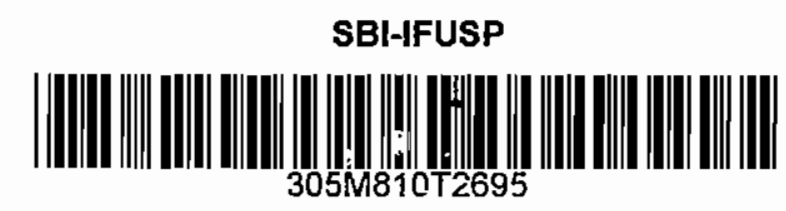

UNIVERSIDADE DE SÃO PAULO

INSTITUTO DE FÍSICA

\title{
TEORIA DO CONFINAMENTO DE BURACOS EM HETEROESTRUTURAS SEMICONDUTORAS DO TIPO $\delta$-DOPING
}

Guilherme Matos Sipahi

Banca Examinadora: Prof. Dr. José Roberto Leite (orientador)
Prof. Dr. Amos Troper
Profa. Dra. Euzi C. F. da Silva
Prof. Dr. Gilmar E. Marques Prof. Dr. Marcos H. Degani

Tese apresentada ao Instituto de Física da Universidade de São Paulo para a obtenção do título de Doutor em Ciências

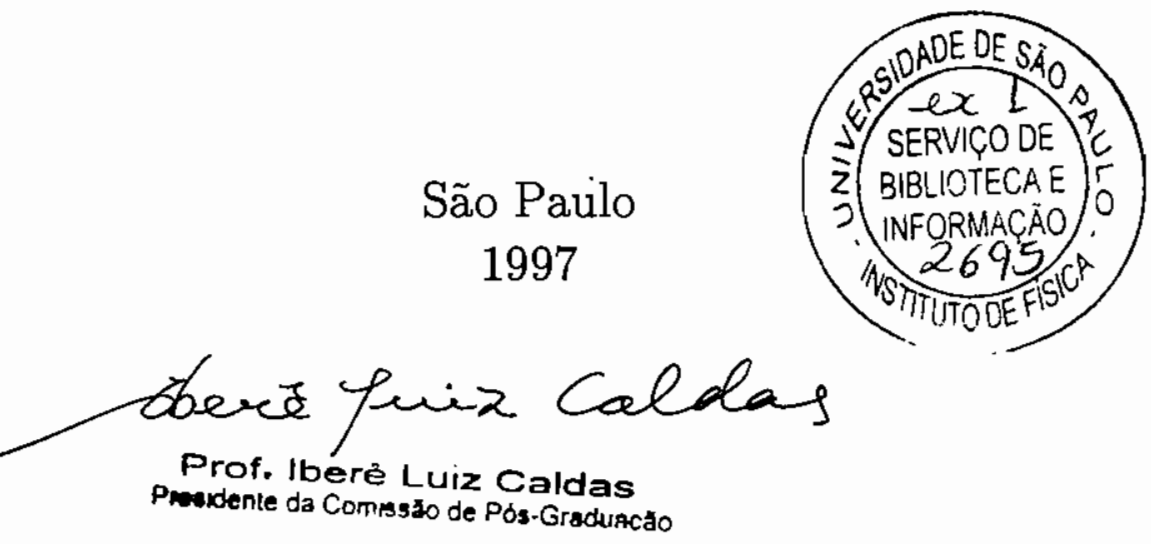




$$
\begin{gathered}
537.622 \\
5618 t \\
D \\
e x .1
\end{gathered}
$$

FICHA CATALOGRÁFICA

Preparada pelo Serviço de Biblioteca e Informação do Instituto de Física da Universidade de São Paulo

Sipahi, Guilherme Matos

Teoria do Confinamento de Buracos em Heteroestruturas Somicondutoras do Tipo 8-Doping. São Paulo, 1997.

Țese (Doutoramento) Universidade de São Pąùlo. Instituto de Física - Departamento de Física dos Materiais o Mocânica.

Área de Concentração: Física do Estado Sólido Orientador: Prof. Dr. José Roberto Leite

Unitermos: 1. $\delta$-Doping; 2. Heteroestruturas;

3. GaAs; 4. Estrutura de Bandas.

USP/IF/SBI - 48/97 


\section{Agradecimentos}

Qualquer um que tenha concluído uma Tese de Doutoramento sabe que, sem o auxílio de muitos, não seria possível concluir um trabalho deste porte.

A meus pais, Aytan e Helenita, e irmãos, Fabiano e Isabel, por seu apoio inconteste.

A'o Prof. José Roberto Leite pela orientação e apoio constantes.

Ao Prof. Rolf Enderlein pela co-orientação e pelas muitas sugestões e discussões.

À Profa. Luísa Maria Ribeiro Scolfaro pela colaboração constante desde o início deste trabalho.

Ao Prof. Dan Lerner da Escola Nacional de Química de Montpellier e ao prof. Henry Mathieu e demais professores do Grupo de Estudos de Semicondutores de Montpellier por me acolherem em minha estada de um ano na França, onde obtive meu D.E.A.

À Funḍação de Amparo à Pesquisa do Estado de São Paulo (FAPESP), pelo financiamento deste trabalho e da participação nos seguintes congressos: IX ICSMM em Liège, VII IC-SLCS em Amsterdam e XXIII ICPS em Berlim.

Ao Júlio e ao Evandro pela presteza e disposição com que elucidaram minhas dúvidas de informática.

À Profa. Euzi C. F. da Silva e ao Sasha pelas discussões sobre luminescência.

A todos os outros colegas do Laboratório de Novos Materiais Semicondutores que me ajudaram nos problemas relativos a esta tese.

À Enza, com amor. 


\section{Abstract}

$p$-type $\delta$-doping quantum wells and superlattices are semiconductor systems of considerable interest for basic research and device applications. In this work, a method for calculating potentials and band structures of such systems is developed. The method relies on a plane wave expansion of the multiband effective mass equation, uses kinetic energy matrices of any size, and takes exchange correlation into account in a more rigorous way than this was done before. The method is used to calculate potential profiles, subband and miniband structures as well as Fermi level positions for a series of $p$-type $\delta$-doping quantum wells and superlattices. The differences between $n$ - and $p$-type $\delta$-doping structures are studied. In addition to this we developed a procedure within this method to calculate photoluminescence (PL) spectra of the wells studied. It depends on the oscillator strength between the holes inside the wells and the free electrons on the conduction band. We use this procedure to calculate envelope functions, overlap integrals and direct and indirect transitions spectra. Finally, we compare our theoretical calculations of PL spectra with experimental results extracted from the literature. 


\section{Resumo}

Poços e super-redes $\delta$-doping tipo são sistemas semicondutores de interesse considerável tanto para a pesquisa básica como para aplicações em dispositivos. Neste trabalho desenvolvemos um novo método para o cálculo de potenciais e estruturas de bandas deste tipo de sistemas. O método baseia-se na expansão em ondas planas da equação da massa efetiva multibandas, usa matrizes de energia cinética de qualquer tamanho e leva em conta o potencial de troca e correlação de uma maneira mais rigorosa do que em trabalhos anteriores. São calculados perfis de potencial e estrutura de minibandas e subbandas bem como a posição do nível de Fermi de uma série de poços isolados e super-redes $\delta$-doping tipo p. São estudadas também as diferenças entre super-redes $\delta$-doping tipo $\mathrm{p}$ e tipo $\mathrm{n}$. A partir deste método foi desenvolvido ainda um procedimento de cálculo de espectros de fotoluminescência dos poços estudados. Este procedimento baseia-se nas forças de oscilador das transições entre os buracos confinados no interior do poço e os elétrons livres da banda de condução. Ele é utilizado para calcular funções envelope, integrais de superposição e espectros de transições diretas e indiretas. Por fim, comparamos espectros calculados teoricamente com resultados experimentais extraídos da literatura. 


\section{Índice}

1 Introdução 1

1.1 Teorias de confinamento de buracos ......... 2

1.2 Fotoluminescência em poços $\delta$-doping tipo p . . . . 5

1.3 Desafios .................... 8

1.4 Este trabalho . . . . . . . . . . . . 10

2 Método $\mathbf{k} \cdot \mathbf{p} \quad 13$

2.1 Hamiltoniano $\mathbf{k} \cdot \mathbf{p} \ldots \ldots \ldots \ldots$

2.2 Teoria de perturbações e funções envelope $\ldots . . . . .17$

2.3 Teoria de perturbações - Bandas não-degeneradas . . . 18

2.3.1 Hamiltoniana não-perturbada . . . . . . . . . 19

2.3.2 Hamiltoniana perturbada e funções envelope . . . 20

2.4 Teoria de perturbações - Bandas degeneradas . . . . . . 22

2.4.1 Hamiltoniana não-perturbada . . . . . . . . 23

2.4.2 Hamiltoniana perturbada e funções envelope . . . 23 
2.5 Os Hamiltonianos $\mathbf{k} \cdot \mathbf{p} \ldots \ldots \ldots . \ldots . \ldots . \ldots 24$

2.5.1 Matrizes de Luttinger-Kohn . . . . . . . . 25

2.5.2 Matriz de Kane . . . . . . . . . . . 30

2.6 Determinação dos parâmetros de Kane através de massas efetivas experimentais ............ 33

2.6.1 Definição dos parâmetros de Luttinger no modelo de Kane . . . . . . . . . . . . . . . . . . 34

2.6.2 Massa efetiva em termos dos parâmetros de Kane 36

2.6.3 Relações entre as massas efetivas . . . . . . . 37

2.6.4 Cálculo dos parâmetros de Kane . . . . . . . . 39

3 Cálculo Autoconsistente da Estrutura de Bandas 43

3.1 Célula unitária e primeira zona de Brillouin . . . . . 45

3.2 Densidade de aceitadores, aproximação de Hartree e nível de Fermi . . . . . . . . . . . . . . . . . . 46

3.3 Equação da massa efetiva e termo de energia cinética . . 48

3.4 Potencial coulombiano . . . . . . . . . . . 50

3.5 Potencial de troca e correlação . . . . . . . . . . 52

3.6 Estruturas das matrizes $H_{0}, V_{C}$ e $V_{X C} \ldots \ldots 60$

4 Cálculo de Espectros de Luminescência 64

4.1 Uma expressão geral para a intensidade de luminescência 65

4.2 Estados iniciais e finais . . . . . . . . . . . . 66

4.3 Força de oscilador . . . . . . . . . . . . . 67 
4.4 Probabilidades de ocupação $\ldots \ldots$. . . . . . . 70

4.5 Dependência do 'gap' com a temperatura . . . . . . . 72

4.6 Programa de cálculo da luminescência . . . . . . . . . 73

$\begin{array}{lll}5 & \text { Estrutura de Bandas } & \mathbf{7 4}\end{array}$

5.1 Testes de adequação . . . . . . . . . . . 75

5.1.1 Poços quadrados e super-redes baseadas em heteroestruturas GaAs/GaAlAs . . . . . . . . . 75

5.1.2 Poços $\delta$-doping tipo n .......... 78

5.2 Estruturas $\delta$-doping tipo $\ldots \ldots \ldots . \ldots 81$

5.2 .1 Potenciais . . . . . . . . . . 81

5.2.2 Estrutura de bandas ........... 84

5.3 Comparação com resultados de outros autores para poços $\delta$-doping tipo $\mathrm{p}$ isolados $\ldots \ldots . \ldots . \ldots 89$

5.4 Comparação entre poços $\delta$-doping tipo p e tipo $n \ldots 91$

6 Fotoluminescência de Estruturas $\delta$-doping tipo p em GaAs

6.1 Super-rede típica . . . . . . . . . . . . 996

6.1.1 Potencial autoconsistente e estrutura de bandas . 96

6.1 .2 Funções envelope . . . . . . . . . . . . 998

6.1 .3 Forças de oscilador . . . . . . . . . . . 100

6.1.4 Espectros de fotoluminescência . . . . . . . . 102 
Índice

6.2 Espectros de fotoluminescência para diversas super-redes $\delta$-doping tipo p . . . . . . . . . . . . 104

6.3 Dependência do espectro com a temperatura . . . . . 106

7 Conclusão

7.1 O trabalho desenvolvido . . . . . . . . . 113

7.2 Principais contribuições . . . . . . . . . . . . . 114

7.3 Perspectivas . . . . . . . . . . . . . 115

A Programas desenvolvidos

A.1 Cálculo da estrutura de bandas . . . . . . . . . 117

A.2 Cálculo do espectro de luminescência . . . . . . . . 120

A.3 Determinação das componentes do potencial . . . . . . . 121 


\section{Lista de Figuras}

2.1 Desenho esquemático da estrutura de bandas do GaAs volumétrico . . . . . . . . . . . . . . 14

3.1 Primeira Zona de Brillouin . . . . . . . . . . . 46

3.2 Estrutura da matriz $H_{\mathbf{k} \cdot \mathbf{p}} \ldots \ldots \ldots 61$

3.3 Estrutura da matriz $V_{C} \ldots \ldots \ldots 2$

3.4 Estrutura da matriz $V_{X C} \ldots \ldots \ldots 6$

5.1 Estrutura de bandas para um poço quadrado não-dopado e isolado com largura $97 \AA$, e barreira de $300 \mathrm{meV}$. . . . 77

5.2 Estrutura de sub-bandas para um poço quadrado isolado de largura $112 \AA$, barreira de $280,6 \mathrm{meV}$ e concentração de portadores $N_{s}=2 \times 10^{11} \mathrm{~cm}^{-2} \ldots \ldots \ldots 78$

5.3 Perfis de potencial para um poço $\delta$-doping tipo $n$ calculados com os dois diferentes métodos . . . . . . . 80

5.4 Perfis de potencial para buracos para super-redes $\delta$-doping tipo p com período $d=500 \AA \ldots . \ldots 2$

5.5 Potenciais total e parcial para buracos numa super-rede $\delta$-doping tipo $\mathrm{p}$ de período $500 \AA \ldots \ldots$ 
5.6 Estruturas de bandas e níveis de Fermi para super-redes $\delta$-doping tipo p com três diferentes concentraçōes de aceitadores ................. 86

5.7 Fundos de poço, estados de buracos e energias de Fermi para super-redes de poços $\delta$-doping tipo p com período de $500 \AA$, desenhadas em função do valor de $\Delta z \ldots 88$

5.8 Perfis de potencial para buracos para super-redes com período $d=500 \AA$ e poços isolados $\delta$-doping tipo $\mathrm{p}$ com concentração de dopantes $N_{s}=8 \times 10^{12} \mathrm{~cm}^{-2} \ldots 90$

5.9 Perfis de potencial, níveis de energia das minibandas e nível de Fermi para super-redes $\delta$-doping tipo p e tipo $\mathrm{n}$ com período de $500 \AA$ e diferentes concentrações . . . . . 93

6.1 Potencial autoconsistente para super-rede com $\mathrm{d}=500 \AA$, $N_{A}=8 \times 10^{12} \mathrm{~cm}^{-2}$ e $\Delta z=23.5 \AA \ldots \ldots 96$

6.2 Curvas de dispersão para sistema da fig. 6.1 . . . . . . 97

6.3 Funções de onda ao quadrado para o mesmo sistema da fig. $6.1 \ldots \ldots . \ldots . \ldots 99$

6.4 Forças de oscilador para o mesmo sistema da fig. 6.1 . . . 101

6.5 Espectros calculados de fotoluminescência para o mesmo sistema da fig. $6.1 \ldots \ldots . \ldots . . \ldots 102$

6.6 Espectros de fotoluminescência teóricos e experimentais para super-redes $\delta$-doping tipo p com período de $600 \AA$ e várias concentrações . . . . . . . . . . . 105

6.7 Espectros de fotoluminescência teóricos e experimentais para super-redes $\delta$-doping tipo p com mesmo período, dispersão e concentração a diferentes temperaturas . . . 106

6.8 Estudo da inclusão de portadores fotocriados em espectros teóricos de poços isolados . . . . . . . . . . . . 108 
6.9 Espectros teóricos e experimentais para poços $\delta$-doping tipo $\mathrm{p}$ isolados. As intensidades são apresentadas em escala logarítmica . . . . . . . . . . . . . . 110 


\section{Lista de Tabelas}

2.1 Parâmetros do modelo de Kane calculados para diferentes conjuntos de massas efetivas . . . . . . . . . . 44 


\section{Capítulo 1}

\section{Introdução}

Semicondutores com dopagem planar, ou ' $\delta$-doping', tipo $\mathrm{p}$ são sistemas de grande interesse tanto para a pesquisa básica quanto para a aplicada ${ }^{[1]}$. Quando comparados com estruturas $\delta$-doping tipo ${ }^{[1-22]}$ representam novos e interessantes desafios ${ }^{[23-31]}$. Apesar do Berílio ter sido largamente usado como dopante tipo $\mathrm{p}$ em crescimento epitaxial de GaAs desde o trabalho original ${ }^{[32]}$ na década de setenta, só no começo dos anos noventa surgem na literatura poços $\delta$-doping de Berílio em $\mathrm{GaAs}^{[23-29]}$. Schubert et al. ${ }^{[2]}$ demonstraram, através de Espectroscopia de Massa de Íon Secundário (SIMS) e medidas de CapacitânciaVoltagem (CV), que as impurezas, nesse tipo de estrutura, podem ter uma dispersão menor que $20 \AA$ em torno da camada de dopagem de cargas. Medidas dessa dispersão em amostras de poços $\delta$-doping de Be:GaAs foram feitas através de Microscopia de Tunelamento Eletrônico (STM) ${ }^{[33]}$, concluindo que para concentrações menores do que $1 \times 10^{13} \mathrm{~cm}^{-2}$, seu valor pode ser menor que $10 \AA$. Assim, mesmo para uma concentração razoavelmente alta de impurezas aceitadoras (da ordem de $10^{13} \mathrm{~cm}^{-2}$ ) garante-se o confinamento de buracos no sistema. Efeitos desse confinamento são observados em espectros de fotoluminescência (PL) ${ }^{(26-30]}$.

Para uma observação de espectros de luminescência é necessária a superposição espacial das funções de onda dos estados de elétrons e bu- 
racos. Para que as funções de onda dos estados do par elétron-buraco recombinante se superponham de maneira mais efetiva são crescidas amostras com poços $\delta$-doping entre duas barreiras ${ }^{[2,28]}$, confinando, dessa maneira, também os portadores minoritários. Os casos mais comuns são os de poços $\delta$-doping de Si:GaAs (tipo n) e de Be:GaAs (tipo $\mathrm{p}$ ) entre barreiras de (Ga,Al)As. Foram observados sinais de PL em sistemas de poços $\delta$-doping tipo p de Be:GaAs ${ }^{[27-29]}$ e C:GaAs ${ }^{[30]}$ sem a presença de barreiras, ou seja, em poços isolados. Em sistemas tipo n nunca foi claramente observado sinal de luminescência sem confinamento dos portadores minoritários entre barreiras. $O$ fato de haver picos associados a poços $\delta$-doping tipo p em espectros de luminescência, por si só já justifica um estudo mais aprofundado do assunto.

\subsection{Teorias de confinamento de buracos}

Do ponto de vista teórico, o tratamento de buracos em poços $\delta$-doping é mais difícil que o tratamento dos elétrons. As principais razōes para tal são: o gás ser composto por diferentes tipos de buracos, leves e pesados principalmente, além dos da banda 'split-off' e de ter também uma relação de dispersão mais complicada que a dos gás de elétrons (não-parabólica e anisotrópica). Outro fator complicante é o fato de os efeitos de troca e correlação serem mais importantes para buracos em poços $\delta$-doping do tipo p que para elétrons em poços $\delta$-doping do tipo n. O tratamento usualmente dado à interação de troca e correlação em sistemas tipo $\mathrm{n}^{[34]}$, baseado no método do funcional de densidade $\mathrm{e}$ na aproximação de densidade local (LDA), não pode ser aplicado indiscriminadamente a sistemas tipo $\mathrm{p}$, porque o potencial de troca e correlação depende da distribuição total de cargas no sistema, englobando indistintamente os dois tipos de portadores. Apesar da correlação coulombiana atuar sobre todos os portadores do sistema sem fazer distinção quanto ao tipo de cada um, a troca atua seletivamente, relacionando somente portadores do mesmo tipo. Em poços $\delta$-doping tipo $\mathrm{p}$, além de haver dois tipos de portadores, suas concentrações são altas. Torna-se então, imprescindível um aprimoramento da aproximação levando em conta os diferentes tipos de portadores. 
Estudos teóricos de sistemas $\delta$-doping tipo $\mathrm{p}$ têm sido feitos desde o início desta década. Cálculos autoconsistentes de estrutura de bandas ${ }^{[28,35]}$ foram realizados usando-se a equação de massa efetiva multibandas de Luttinger-Kohn em torno do ponto $\Gamma^{[36]}$. Utilizaram-se nesses trabalhos métodos desenvolvidos uma década antes $(1984,1985)$ para o tratamento do problema do confinamento de buracos em uma heterointerface de GaAs/(Al,Ga)As ${ }^{[37-40]}$ e também em poços e superredes do mesmo material ${ }^{40-42]}$. Para trabalhar com buracos em heterointerfaces esses últimos autores se basearam em trabalhos sobre buracos em canais $\mathrm{p}$ de camadas de inversão em $\mathrm{Si} / \mathrm{SiO}_{2}$, um problema resolvido ainda uma década mais cedo (1975) por Bangert, von Klitzing e Landwehr ${ }^{[43,44]}$ e por Okhawa e Uemura ${ }^{[45]}$. Também foi usado o trabalho de Nedorezov ${ }^{[46]}$ que resolveu o problema do confinamento de buracos em um filme fino (um poço com barreiras infinitamente altas), bem como o trabalho desenvolvido sobre níveis aceitadores rasos a partir do artigo inicial de Luttinger e Kohn em $1955^{[36]}$.

O uso do modelo de Luttinger-Kohn para o operador de energia cinética para buracos, ou mais precisamente, de sua versão simplificada $(4 \times 4)$, tornou-se usual no campo. A equação da função envelope correspondente foi explicitada e resolvida por diferentes métodos e aproximações. À exceção de Nedorezov ${ }^{[46]}$, que achou uma solução analítica exata, todos os outros autores usaram alguma forma de procedimento variacional, e muitos omitiram a anisotropia axial (usando a, assim chamada, aproximação axial). As funções auxiliares para o procedimento variacional eram escolhidas intuitivamente ou obtidas, analítica ou numericamente, resolvendo-se uma equação de função envelope auxiliar para uma única componente, escolhida para ser a mais parecida possível com a equação de Luttinger-Kohn com 4 componentes. As funções auxiliares eram usadas como base para a representação matricial do Hamiltoniano de Luttinger-Kohn. Os autovalores e autovetores da matriz correspondente podiam então ser calculados através de parâmetros variacionais, sendo estes depois otimizados utilizando-se o princípio variacional.

Os diversos trabalhos em confinamento de buracos diferem entre si a respeito das formas de solução (em particular quanto à função auxiliar usada), à maneira como essa equação é resolvida, ao tamanho da base 
usada para a representação matricial do Hamiltoniano e às outras aproximações adicionais necessárias ao Hamiltoniano. Eles também diferem no tratamento da interação de troca e correlação entre buracos. Vamos agora apresentar as soluções dadas nos dois trabalhos sobre poços $\delta$-doping descritos abaixo.

Os autores da ref. [35] usam uma versão melhorada do método de Broido e Sham ${ }^{[38]}$. Uma transformação unitária independente de $z$ é feita de modo a chegar-se numa base na qual o Hamiltoniano compõe-se de dois subespaços desacoplados, um para spins positivos e outro para spins negativos, os quais são degenerados devido à combinação simetria de reversão temporal e de inversão espacial. Os subespaços são ainda uma combinação de estados de buracos leves e pesados. Os elementos nãodiagonais do Hamiltoniano de Luttinger-Kohn em cada subespaço são responsáveis pela não-parabolicidade e pela anisotropia da estrutura de bandas. Apesar de não podermos desprezá-las, podemos levá-las em conta passo a passo. Num primeiro passo os autovalores e autovetores dos 2 blocos $2 \times 2$ independentes são calculados, o que é feito usando-se uma representação de ondas planas das funções dessas matrizes. As autofunções calculadas numericamente são tomadas como bases para a matriz da representação do Hamiltoniano de Luttinger-Kohn completo. A diagonalização da matriz de Luttinger-Kohn completa revela a estrutura das sub-bandas de buracos e as autofunções correspondentes. A aproximação axial não é feita neste caso.

O procedimento apresentado na ref. [28] é praticamente o mesmo feito por Ekenberg e Altarelli em interfaces $\mathrm{GaAs} /(\mathrm{Al}, \mathrm{Ga}) \mathrm{As}^{[37]}$. São utilizadas funções Gaussianas auxiliares, com 2 parâmetros livres por função. O Hamiltoniano de Luttinger-Kohn é substituído por uma matriz 2x2 com base nos mesmos argumentos do procedimento anterior. Usa-se também a aproximação axial, ou seja, despreza-se o termo de simetria não-axial. O Hamiltoniano é representado na base das funções auxiliares e a matriz obtida é diagonalizada. Como os autores não especificam quantas funções usam na base, é difícil determinar a precisão de seus resultados.

Em qualquer sistema em que haja confinamento de buracos é funda- 
mental tratar a estrutura da banda de valência em sua complexidade. A interação entre buracos, porém, somente é essencial nos casos em que sua concentração cause modificações perceptíveis no potencial total do sistema. Não se pode tratar camadas de inversão, heterointerfaces simples ou estruturas $\delta$-doping, sem levá-la em conta. Para níveis aceitadores de poços quânticos e super-redes não-dopados baseados em heteroestruturas, porém, pode-se omití-la.

Ao estudar canais p em camadas de inversão, usando uma versão simplificada da estrutura da banda de valência e do método da densidade local, Okhawa ${ }^{[47]}$ demonstrou que, ao contrário dos elétrons, a troca e a correlação atuam de maneira fundamental no caso de buracos. As massas efetivas maiores e, por consequiência, a maior densidade local são seus principais responsáveis. Resultados similares foram obtidos para o silício por Ando ${ }^{[48]}$. Resultados menos rigorosos sobre a importância dos efeitos de muitos corpos em GaAs e outros materiais do tipo 'ZincBlende' são apresentados nas refs. [35,40,49,50]. Ando em seu trabalho sobre heterojunções $\mathrm{GaAs} /(\mathrm{Al}, \mathrm{Ga}) \mathrm{As}^{[40]}$ afirma que os efeitos de muitos corpos da interação de troca e correlação são fundamentais, mas não os leva em conta. Por essas razões, qualquer tratamento rigoroso de confinamento de buracos nessas estruturas não pode deixar de levar em conta efeitos de troca e correlação, como foi feito na ref. [28]. A maneira como esses efeitos foram incluídos em [35] representa no entanto uma primeira aproximação. É necessário mais trabalho para tratar este problema adequadamente.

\subsection{Fotoluminescência em poços $\delta$-doping tipo $p$}

As propriedades ópticas podem ser utilizadas para se estudar a localização de portadores em uma região de uma amostra. A recombinação de portadores majoritários, localizados nos poços, com portadores minoritários presentes na mesma região da amostra gera picos no espectro de luminescência em energias em geral menores que a do material vo- 
lumétrico ('bulk'). O fato de os portadores precisarem estar na mesma região da amostra limita o uso desta técnica. Em estruturas $\delta$-doping tipo n em GaAs, essa limitação é explicitada ${ }^{[8,9,51,52]}$. Não se vê, em espectros de PL, estruturas derivadas da recombinação de pares dos elétrons confinados nos poços com buracos da banda de valência. A explicação é simples. O potencial gerado pela camada de doadores em poços $\delta$-doping tipo n é repulsivo para buracos, portanto esses são expulsos da região do poço. Por conseqüência as funções de onda de buracos e elétrons na prática não se superpõem e não há possibilidade de recombinação de pares. Para que a superposição das funções de onda se produza efetivamente, coloca-se o poço $\delta$-doping tipo n entre duas barreiras de potencial de forma a localizar os buracos em um poço, isto é, o espaço entre as duas barreiras. Com esse tipo de sistema os espectros de PL podem ser utilizados para o estudo das propriedades dos portadores confinados no poço $\delta$-doping (elétrons nesse caso).

Os trabalhos das refs. [26] e [28] mostram resultados de medidas de $\mathrm{PL}$ em poços $\delta$-doping de Be:GaAs entre barreiras de $(\mathrm{Al}, \mathrm{Ga}) \mathrm{As}$ e o trabalho da ref. [30] uma super-rede de poços $\delta$-doping de C:GaAs. A idéia é a mesma, confinar os portadores minoritários (neste caso elétrons) entre as barreiras ou na região entre poços consecutivos, de forma a aumentar a superposição entre os estados de elétrons e buracos e favorecer a recombinação dos mesmos. De acordo com as refs. [27] e [29], no entanto, as amostras utilizadas nas medidas são poços $\delta$ doping de Be:GaAs isolados e apresentam espectros com picos na faixa de energia de recombinação dos pares elétron-buraco.

Apesar das diferenças entre os diversos sistemas o que se observa (e que se assume que seja devido à luminescência de estados dentro do poço), é bastante consistente. Uma estrutura larga em cujo extremo superior encontra-se um pico (aprox. de $1500 \mathrm{meV}$, ou seja, cerca de $20 \mathrm{meV}$ abaixo do 'gap') e que apresenta uma cauda no espectro, a qual se estende por várias dezenas, ou mesmo uma centena, de meV. Nos espectros mais bem definidos vê-se claramente um segundo pico 30 a $40 \mathrm{meV}$ abaixo do primeiro. Nos espectros de poços $\delta$-doping isolados, vê-se um pico na posição esperada para o éxciton. A semelhança entre os espectros dos diversos sistemas reitera a observação nos casos 
de espectros de poços isolados de estruturas relacionadas com estados dentro do mesmo.

Em virtude da presença de luminescência em poços isolados como os descritos nas refs. [27] e [29], faz-se necessário revisar o modelo de luminescência descrito anteriormente. Existem diferenças fundamentais entre poço $\delta$-doping tipo $\mathrm{p}$ e tipo n no que concerne à luminescência. Em virtude da maior localização dos buracos e portanto de uma maior blindagem do potencial coulombiano por estes, os poços tipo p são mais rasos que seus congêneres tipo n. Os elétrons, os portadores minoritários em poços tipo $\mathrm{p}$, têm funções de onda mais delocalizadas que os buracos, portadores minoritários em poços tipo n. Esses dois fatores se superpõem, fazendo com que a integral de superposição entre portadores minoritários e majoritários seja muito maior em poços tipo $\mathrm{p}$ que em poços tipo $\mathrm{n}$. Junto a esta diferença entre poços tipo $\mathrm{p}$ e tipo n, uma segunda hipótese ${ }^{[27,53]}$ deve ser também levada em conta. O laser incidindo sobre a amostra dá origem a um certo número de pares elétron-buraco dissociados que enquanto não se recombinam, estão sujeitos à ação do potencial da amostra. Os buracos são atraídos para o poço, gerando um excesso de cargas em seu interior que se reflete no aparecimento, no topo de sua barreira, de um pequeno morro atrativo para os elétrons. Esse morro forma um poço raso que confina os elétrons fotogerados, os quais com o aumento da temperatura da amostra devem tornar-se livres de novo. Os picos de luminescência são decorrentes da recombinação dos elétrons confinados ou de elétrons quase livres próximos ao fundo da banda de condução com os buracos dentro do poço, tendo conseqüentemente energia inferior à do 'gap'. As duas hipóteses devem ser estudadas.

\subsection{Desafios}

Após a apreciação deste panorama experimental e teórico percebe-se a necessidade do aprimoramento das investigações do comportamento dos buracos em estruturas $\delta$-doping tipo p, sejam elas poços isolados ou super-redes. Este é um problema apenas parcialmente resolvido e al- 
guns desafios e oportunidades interessantes se colocam. Primeiro, diferentemente de poços e super-redes baseados em heteroestruturas apenas o operador do potencial da equação de massa efetiva é espacialmente inomogêneo. Por ser um sistema de composição homogênea, o operador de energia cinética é constante no espaço. Isto significa que a equação da massa efetiva é aplicável a todos os pontos do sistema, ao contrário do caso de heteroestruturas em que ela é aplicável apenas a camadas homogêneas de material. Isto leva à necessidade da aplicação de condições de contorno nas interfaces. Há na literatura diferentes propostas para estas soluções e os erros introduzidos podem ser incontroláveis ${ }^{[54]}$. A teoria da massa efetiva para poços e super-redes $\delta$-doping não contém tais incertezas. Além disso, o problema de autovalores pode ser resolvido por método matricial, sem restrições para a escolha das funções da base. No caso de heteroestruturas essas restrições resultam da representação do operador de energia cinética, que por sua vez, depende de uma base de funções de onda diferentes para cada material. Para estruturas $\delta$-doping, qualquer conjunto de funções de base razoável pode ser usada. Ondas planas são particularmente desejáveis por duas razões: o operador de energia cinética é expresso originalmente nessa base, e a equação de Poisson se transforma numa expressão algébrica simples quando escrita na mesma.

Segundo, o potencial dos buracos de estruturas $\delta$-doping é essencialmente determinado pelo próprio gás, diferentemente das heteroestruturas onde o gás introduz apenas pequenas correções ao potencial, o qual é, em sua maior parte, determinado pelas descontinuidades das bandas. A interação entre buracos exerce então um papel fundamental nesse tipo de estruturas. Mesmo que ocorra uma situação análoga em estruturas $\delta$-doping do tipo n, os dois tipos de sistemas têm comportamentos bastante diferenciados. As concentrações experimentais típicas de estruturas $\delta$-doping para o tipo p são quase uma ordem de grandeza maiores que para o tipo n. Com base nestas indicações poder-se-ia argumentar que os efeitos de troca e correlação devem ser menos importantes para os primeiros. Isto é verdade quando comparamos esses efeitos ao potencial repulsivo, gerado pelos portadores, o chamado potencial de Hartree. Este último é, no entanto, apenas parte do potencial de uma partícula total sem troca e correlação. O potencial atrativo devido ao 
plano de dopantes tem ainda de ser adicionado ao primeiro para termos este último. Devido à forte localização dos buracos essas duas partes do potencial devem se compensar mais efetivamente em estruturas tipo $\mathrm{p}$ do que em estruturas tipo $\mathrm{n}$. Por outro lado, o termo de troca e correlação do potencial depende, na aproximação de densidade local, apenas da densidade dos portadores que deve ser mais alta em virtude da maior localização dos buracos. Ao examinarmos esses aspectos do problema, percebe-se que os efeitos de troca e correlação devem ter um papel particularmente importante em estruturas $\delta$-doping tipo p. Surge daí a necessidade de um tratamento mais acurado desses efeitos, se comparado ao procedimento que toma o potencial de troca e correlação do gás de buracos de Luttinger-Kohn como uma matriz diagonal com os potenciais de troca e correlação de buracos leves e pesados como elementos diagonais ${ }^{[35]}$.

Terceiro, poços e super-redes $\delta$-doping têm caráter metálico. Ao calcularse autoconsistentemente os potenciais de Hartree, a soma sobre os estados ocupados deve levar em conta todos os pontos $\mathbf{k}$ abaixo do nível de Fermi ao invés de levar em conta apenas pontos especiais, como é o caso em semicondutores com bandas totalmente ocupadas ${ }^{[55]}$. Para elétrons em um poço $\delta$-doping pode-se tomar a dispersão das bandas como isotrópica e parabólica na direção paralela à camada de impurezas, o que torna possível fazer essa soma analiticamente. A aproximação isotrópica não é, no entanto, aplicável aos buracos e essa soma deve ser feita numericamente. Isto representa um procedimento custoso, em termos de tempo de processamento, porém inevitável .

Quarto e último, diferentemente de super-redes e poços quânticos de heteroestruturas baseadas em $\mathrm{GaAs} /(\mathrm{Ga}, \mathrm{Al}) \mathrm{As}$ nas super-redes e poços do tipo $\delta$-doping, apenas um tipo de portadores sente os poços e é confinado. Como já foi dito, os portadores minoritários sentem uma barreira de potencial e são espalhados por ela. Estes portadores têm funções de onda que se espalham por toda a estrutura, com exceção daqueles que têm energias abaixo da barreira, cuja região é proibida. Então, as probabilidades de transição ópticas entre os estados estendidos e confinados de elétrons e buracos são funções contínuas das energias e que variam fortemente. Esse comportamento mostra-se completamente diferente 
do encontrado em heteroestruturas baseadas em GaAs/(Ga, $\mathrm{Al}) \mathrm{As}$, aonde não existe essa dependência em energia. Portanto para o cálculo de espectros óticos e de luminescência de estruturas $\delta$-doping necessita-se das funções de onda de elétrons e de buracos obtidas numericamente. Pode-se calculá-las através de modelos independentes para elétrons e buracos, mas essa não é a solução ideal. O mais apropriado seria calculá-las através de um modelo aplicável simultaneamente a ambos, elétrons e buracos ao invés de um modelo para buracos e a equação da massa efetiva de uma banda para elétrons. Então, para se calcular o espectro de luminescência de poços $\delta$-doping faz-se necessário o uso do modelo de Kane, mesmo para o GaAs, um caso bem descrito pelo Hamiltoniano de Luttinger-Kohn. Para que o esforço numérico se mantenha em limites razoáveis necessita-se de um procedimento simples para a solução da equação de massa efetiva. O método matricial com expansão das funções em ondas planas satisfaz essa condição.

\subsection{Este trabalho}

Pode-se dividir este projeto em duas partes distintas. Numa primeira etapa foi desenvolvido um método teórico para o cálculo numérico da estrutura de bandas de estruturas baseadas em poços $\delta$-doping em GaAs. Utilizamos a resolução autoconsistente da equação de massa efetiva multibandas no espaço recíproco, usando uma expansão da mesma em ondas planas. Para o operador de energia cinética utilizamos as matrizes dos modelos $\mathbf{k} \cdot \mathbf{p}$ de Luttinger-Kohn $(4 \times 4)$ e de Kane $(8 \times 8)$. Para escrever o operador de energia potencial coulombiana foi usada a integral segunda da equação de Poisson para uma distribuição de cargas gerada através da aproximação de Hartree. Para o operador da energia potencial de troca e correlação foi utilizada uma generalização do potencial proposto por Hedin e Lundqvist ${ }^{[34]}$ para dois tipos diferentes de portadores $^{[56]}$. Para tanto parte-se do pressuposto, já enunciado no artigo original de Hedin e Lundqvist, de que o termo de troca e correlação do potencial total pode ser separado em dois termos independentes, um que se refere à correlação de troca e outro que se refere à correlação coulombiana. A distribuição de cargas dos portadores pode ser sepa- 
rada em distribuições diferentes para cada tipo de portador. Como a interação de troca opera apenas sobre o mesmo tipo de portador e a correlação coulombiana não faz distinção, deve-se usar para calcular o termo de troca, a distribuição de carga específica do tipo de portador a que ela se refere, e para calcular o termo de correlação, a distribuição de cargas total da amostra. De posse desses potenciais diferenciados para cada tipo de portador pode-se escrever um potencial diagonal. um estudo da simetria do sistema levou à proposição, ainda, de dois outras formas para a matriz de troca e correlação. As três diferentes formas da matriz foram testadas para encontrar-se a mais adequada.

A segunda etapa consistiu no desenvolvimento de um modelo para o cálculo numérico de espectros de luminescência utilizando o programa derivado do método de cálculo da estrutura de bandas desenvolvido. Duas versões diferentes foram feitas. A primeira utiliza apenas transições diretas, enquanto que a segunda utiliza, além das transições diretas, transições indiretas.

Os programas baseados nesses modelos foram desenvolvidos em FORTRAN77 e são portáveis para diferentes plataformas. Atualmente utilizamos para executar os programas de cálculo da maioria dos sistemas, microcomputadores Pentium com 64 Mbytes de memória RAM e os sistemas operacionais Linux ou Windows. Para os que têm maior necessidade memória RAM ou de tempo de cálculo, dispomos ainda de uma estação de trabalho DEC-Alpha com 256 Mbytes de memória RAM rodando em OSF/Unix. Os programas de cálculo foram devidamente testados e comparados com resultados presentes na literatura.

O método $\mathbf{k} \cdot \mathbf{p}$ será descrito no capítulo 2 , o método de cálculo da estrutura de bandas será descrito no capítulo 3 e o método para o cálculo da luminescência no capítulo 4 . No capítulo 5 apresentaremos os resultados dos cálculos da estrutura de bandas, bem como os testes para avaliar o método utilizado. Neste capítulo serão apresentados gráficos dos potenciais dos poços, das estruturas das bandas de valência e de condução. Serão apresentados como testes comparações dos resultados aqui obtidos com resultados clássicos da literatura e ainda testes de convergência da aproximação por ondas planas. No capítulo 6 apre- 
sentaremos os resultados dos cálculos de espectros de luminescência e comparações dos mesmos com resultados da literatura. No capítulo 7 serão feitas as consideraçōes finais e o apêndice será reservado a descrições sucintas dos programas desenvolvidos. 


\section{Capítulo 2}

\section{Método $\mathbf{k} \cdot \mathbf{p}$}

Para o estudo que faremos sobre as estruturas baseadas em poços $\delta$ doping tipo $\mathrm{p}$, consideraremos as mesmas como perturbações ao semicondutor volumétrico ('bulk'). Este último será tratado segundo a teoria da massa efetiva, na aproximação $\mathbf{k} \cdot \mathbf{p}$. Antes de introduzirmos este método é apropriado descrevermos a estrutura de bandas do GaAs volumétrico.

A fig. 2.1 apresenta um desenho esquemático das bandas de condução e de valência do GaAs e suas simetrias. O ponto de mais alta simetria é o ponto $\Gamma(\mathbf{k}=0)$, o centro da Zona de Brillouin do sistema. Sem a inclusão da interação spin-órbita, a banda de condução é bidegenerada (spin 'up' e spin 'down'), com simetria $\Gamma_{1}$ e a banda de valência é hexadegenerada no ponto $\Gamma$, com simetria $\Gamma_{15}$ (fig. 2.1 à esquerda). Nos outros pontos da zona de Brillouin, a banda se subdivide em uma banda quadridegenerada (dois estados com spin 'up' e dois com spin 'down'), chamados de buracos pesados por terem massa efetiva maior, e uma banda bidegenerada (spins 'up' e 'down'), chamados de buracos leves em virtude de sua menor massa efetiva.

A inclusão da interação spin-órbita a este sistema (fig. 2.1 à direita) quebra a simetria da banda de valência, que passa a ter dois valores no ponto $\Gamma$ : uma sub-banda quadridegenerada, com simetria $\Gamma_{8}$ que 


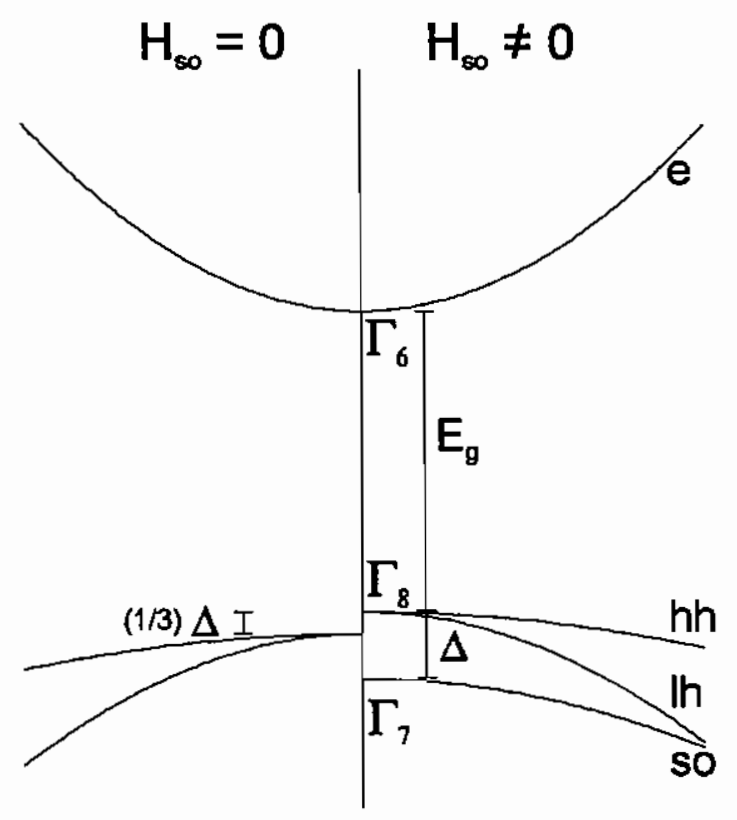

Figura 2.1: Desenho esquemático da estrutura de bandas do GaAs volumétrico.

se subdivide em duas sub-bandas diferentes bidegeneradas (spin 'up' e spin 'down') fora deste ponto (buracos leves e pesados), e uma segunda banda abaixo desta ('split-off'), separada por uma energia $\Delta=$ $340 \mathrm{meV}$, também bidegenerada (spin 'up' e spin 'down'), com simetria $\Gamma_{7}$. A banda de condução permanece bidegenerada (spins 'up' e 'down') e tem simetria $\Gamma_{6}$. A separação ('gap') entre o topo da banda de valência e o fundo da banda de condução tem o valor de $E_{g}=1519,2$ $\mathrm{meV}$ à temperatura de $\mathrm{T}=0 \mathrm{~K}$.

\subsection{Hamiltoniano $\mathrm{k} \cdot \mathrm{p}$}

Para a descrição de sistemas periódicos é necessária a utilização de uma base completa de funções de onda que dê conta de sua periodicidade. A base completa de funções de onda mais utilizada é a base das funções 
de Bloch:

$$
(\mathbf{x} \mid \nu \mathbf{k})_{B l o c h}=\frac{1}{\sqrt{\Omega}} e^{i \mathbf{k} \cdot \mathbf{x}} u_{\nu \mathbf{k}}(\mathbf{x})
$$

onde $\mathbf{k}$ é um vetor da rede recíproca e $\nu$ é o índice da banda.

Nos casos aqui estudados opta-se pela utilização de uma base equivalente. A base das funções de Luttinger-Kohn ${ }^{[36]}$ :

$$
\begin{aligned}
\left(\mathbf{x} \mid \nu \mathbf{k} \mathbf{k}_{0}\right) & =\frac{1}{\sqrt{\Omega}} e^{i \mathbf{k} \cdot \mathbf{x}} u_{\nu \mathbf{k}_{0}}(\mathbf{x})=e^{i\left(\mathbf{k}-\mathbf{k}_{0}\right) \cdot \mathbf{x}} \frac{1}{\sqrt{\Omega}} e^{i \mathbf{k}_{0} \cdot \mathbf{x}} u_{\nu \mathbf{k}_{0}}(\mathbf{x}) \\
& =e^{i\left(\mathbf{k}-\mathbf{k}_{0}\right) \cdot \mathbf{x}}\left(\mathbf{x} \mid \nu \mathbf{k}_{0}\right)_{\text {Bloch }}=e^{i\left(\mathbf{k}-\mathbf{k}_{0}\right) \cdot \mathbf{x}}\left(\mathbf{x} \mid \nu \mathbf{k}_{0} \mathbf{k}_{0}\right)
\end{aligned}
$$

A vantagem da utilização desta base reside na descrição das autofunções de pontos de baixa simetria no espaço recíproco em termos das autofunções de pontos de alta simetria, os pontos $\mathbf{k}_{0}$. Dessa maneira simplifica-se o tratamento das primeiras.

Um cristal perfeito, isto é, um sistema sujeito unicamente ao potencial coulombiano periódico de suas partículas, é descrito pelo seguinte Hamiltoniano:

$$
H=\frac{\mathbf{p}^{2}}{2 m_{0}}+V(\mathbf{x})
$$

onde $V(\mathbf{x})$ é o potencial cristalino periódico e $m_{0}$ a massa de repouso do elétron. As funções de Bloch são autofunções deste Hamiltoniano.

A equaçāo de Schrödinger para um sistema cristalino pode ser escrita como:

$$
\left.H \mid \Psi)=E \mid \Psi)=\sum_{\nu^{\prime} \mathbf{k}^{\prime}} H \mid \nu^{\prime} \mathbf{k}^{\prime} \mathbf{k}_{0}\right)\left(\nu^{\prime} \mathbf{k}^{\prime} \mathbf{k}_{0} \mid \Psi\right)
$$

$\mathrm{Na}$ base das funções de Luttinger-Kohn teremos, para cada componente $\left(\nu \mathbf{k k}_{0} \mid \Psi\right)$ :

$$
E\left(\nu \mathbf{k k}_{0} \mid \Psi\right)=\sum_{\nu^{\prime} \mathbf{k}^{\prime}}\left(\nu \mathbf{k k}_{0}|H| \nu^{\prime} \mathbf{k}^{\prime} \mathbf{k}_{0}\right)\left(\nu^{\prime} \mathbf{k}^{\prime} \mathbf{k}_{0} \mid \Psi\right)
$$


Examinemos agora a matriz $\left(\nu \mathbf{k k}_{0}|H| \nu^{\prime} \mathbf{k}^{\prime} \mathbf{k}_{0}\right)$ :

$$
\begin{aligned}
\left(\nu \mathbf{k k}_{0}|H| \nu^{\prime} \mathbf{k}^{\prime} \mathbf{k}_{0}\right) & =\left(\nu \mathbf{k}_{0}\left|\left[\frac{\mathbf{p}^{2}}{2 m_{0}}+V(\mathbf{x})\right]\right| \nu^{\prime} \mathbf{k}^{\prime} \mathbf{k}_{0}\right) \\
& =\left(\nu \mathbf{k}_{0} \mathbf{k}_{0}\left|e^{-i\left(\mathbf{k}-\mathbf{k}_{0}\right) \cdot \mathbf{x}}\left[\frac{\mathbf{p}^{2}}{2 m_{0}}+V(\mathbf{x})\right] e^{i\left(\mathbf{k}^{\prime}-\mathbf{k}_{0}\right) \cdot \mathbf{x}}\right| \nu^{\prime} \mathbf{k}_{0} \mathbf{k}_{0}\right)
\end{aligned}
$$

Sabendo que $\mathbf{p}=-i \hbar \nabla$ temos que:

$$
\mathbf{p} e^{i\left(\mathbf{k}-\mathbf{k}_{0}\right) \cdot \mathbf{x}}=-i \hbar \nabla e^{i\left(\mathbf{k}-\mathbf{k}_{0}\right) \cdot \mathbf{x}}=\hbar\left(\mathbf{k}-\mathbf{k}_{0}\right) e^{i\left(\mathbf{k}-\mathbf{k}_{0}\right) \cdot \mathbf{x}},
$$

então podemos calcular o comutador entre $\mathbf{p}^{2}$ e $e^{i\left(\mathbf{k}-\mathbf{k}_{0}\right) \cdot \mathbf{x}}$ :

$$
\left[\mathbf{p}^{2}, e^{i\left(\mathbf{k}-\mathbf{k}_{0}\right) \cdot \mathbf{x}}\right]=e^{i\left(\mathbf{k}-\mathbf{k}_{0}\right) \cdot \mathbf{x}}\left[\mathbf{p}^{2}+2 \hbar\left(\mathbf{k}-\mathbf{k}_{0}\right) \cdot \mathbf{p}+\hbar^{2}\left(\mathbf{k}-\mathbf{k}_{0}\right)^{2}\right] .
$$

Portanto:

$$
\begin{aligned}
& \left(\nu \mathbf{k}_{0}|H| \nu^{\prime} \mathbf{k}^{\prime} \mathbf{k}_{0}\right)= \\
& =\left(\nu \mathbf{k}_{0} \mathbf{k}_{0}\left|e^{i\left(\mathbf{k}^{\prime}-\mathbf{k}\right) \cdot \mathbf{x}}\left[\frac{\mathbf{p}^{2}}{2 m_{0}}+V(\mathbf{x})+\frac{\hbar}{m_{0}}\left(\mathbf{k}-\mathbf{k}_{0}\right) \cdot \mathbf{p}+\frac{\hbar^{2}\left(\mathbf{k}-\mathbf{k}_{0}\right)^{2}}{2 m_{0}}\right]\right| \nu^{\prime} \mathbf{k}_{0} \mathbf{k}_{0}\right)= \\
& =\left(\nu \mathbf{k}_{0} \mathbf{k}_{0}\left|\left[\frac{\mathbf{p}^{2}}{2 m_{0}}+V(\mathbf{x})+\frac{\hbar}{m_{0}}\left(\mathbf{k}-\mathbf{k}_{0}\right) \cdot \mathbf{p}+\frac{\hbar^{2}\left(\mathbf{k}-\mathbf{k}_{0}\right)^{2}}{2 m_{0}}\right]\right| \nu^{\prime} \mathbf{k}_{0} \mathbf{k}_{0}\right) \delta_{\mathbf{k k}^{\prime}} \cdot(2.9)
\end{aligned}
$$

Mas as funções de Bloch são autofunções do Hamiltoniano para um potencial periódico $H=\frac{\mathbf{p}^{2}}{2 m_{0}}+V(\mathbf{x})$ :

$$
\left.\left.H \mid \nu \mathbf{k}_{0} \mathbf{k}_{0}\right)=E_{\nu}^{0}\left(\mathbf{k}_{0}\right) \mid \nu \mathbf{k}_{0} \mathbf{k}_{0}\right) .
$$

Assim sendo:

$$
\left(\nu \mathbf{k k}_{0}|H| \nu^{\prime} \mathbf{k}^{\prime} \mathbf{k}_{0}\right)=\left[E_{\nu}^{0}\left(\mathbf{k}_{0}\right) \delta_{\nu \nu^{\prime}}+\frac{\hbar}{m_{0}}\left(\mathbf{k}-\mathbf{k}_{0}\right) \cdot\left(\nu \mathbf{k}_{0} \mathbf{k}_{0}|\mathbf{p}| \nu^{\prime} \mathbf{k}_{0} \mathbf{k}_{0}\right)\right] \delta_{\mathbf{k k}^{\prime}}
$$

onde:

$$
E_{\nu}^{0}(\mathbf{k})=E_{\nu}^{0}\left(\mathbf{k}_{0}\right)+\frac{\hbar^{2}\left(\mathbf{k}-\mathbf{k}_{0}\right)^{2}}{2 m_{0}}
$$


Podemos reescrever esse Hamiltoniano em dois termos distintos:

$$
H_{\mathbf{k} \cdot \mathbf{p}}(\mathbf{k})=H_{0}(\mathbf{k})+\frac{\hbar}{m_{0}}\left(\mathbf{k}-\mathbf{k}_{0}\right) \cdot \mathbf{p}
$$

onde:

$$
\left(\nu \mathbf{k}_{0} \mathbf{k}_{0}\left|H_{0}(\mathbf{k})\right| \nu^{\prime} \mathbf{k}_{0} \mathbf{k}_{0}\right)=E_{\nu}^{0}(\mathbf{k}) \delta_{\nu \nu^{\prime}}
$$

Das expressões obtidas tiramos:

$$
\left(\nu \mathbf{k}_{0}|H| \nu^{\prime} \mathbf{k}^{\prime} \mathbf{k}_{0}\right)=\left(\nu \mathbf{k}_{0} \mathbf{k}_{0}\left|H_{\mathbf{k} \cdot \mathbf{p}}(\mathbf{k})\right| \nu^{\prime} \mathbf{k}_{0} \mathbf{k}_{0}\right) \delta_{\mathbf{k} \mathbf{k}^{\prime}} .
$$

\subsection{Teoria de perturbações e funções en- velope}

Os sistemas que pretendemos estudar são submetidos a outros potenciais periódicos além do potencial cristalino. Esses potenciais são, em geral, muito mais fracos que o próprio potencial cristalino. Quando este é o caso podemos nos valer da teoria de perturbações para tratálos. Nestes casos as autofunções do sistema são as chamadas funções envelope que desenvolveremos a seguir.

Vamos introduzir um novo potencial periódico $U(\mathbf{x})$. Este novo sistema é descrito pelo seguinte Hamiltoniano:

$$
\mathcal{H}=H+U(\mathbf{x})
$$

Onde o potencial perturbativo é:

$$
\left(\nu \mathbf{k}_{0}|U| \nu^{\prime} \mathbf{k}^{\prime} \mathbf{k}_{0}\right)=\left(\mathbf{k}|U| \mathbf{k}^{\prime}\right) \delta_{\nu \nu^{\prime}}
$$

Podemos então escrever a equação de autovalores deste sistema:

$$
E\left(\nu \mathbf{k}_{\mathbf{0}} \mid \Psi\right)=\sum_{\nu^{\prime} \mathbf{k}^{\prime}}\left[\left(\nu \mathbf{k} \mathbf{k}_{0}|H| \nu^{\prime} \mathbf{k}^{\prime} \mathbf{k}_{0}\right)+\left(\mathbf{k}|U| \mathbf{k}^{\prime}\right) \delta_{\nu \nu^{\prime}}\right]\left(\nu^{\prime} \mathbf{k}^{\prime} \mathbf{k}_{0} \mid \Psi\right)
$$


Definindo a função envelope como:

$$
\left(\nu \mathbf{k k}_{0} \mid \Psi\right)=F_{\nu}(\mathbf{k}) \delta_{\nu \nu^{\prime}}
$$

podemos reescrever a equação de autovalores como:

$$
E_{\nu} F_{\nu}(\mathbf{k})=\sum_{\mathbf{k}^{\prime}}\left[E_{\nu}^{n . p .}(-i \nabla) \delta_{\mathbf{k k}^{\prime}}+\left(\mathbf{k}|U| \mathbf{k}^{\prime}\right)\right] F_{\nu}\left(\mathbf{k}^{\prime}\right)
$$

Para termos certeza da correção destas definições voltemos ao espaço direto. Para tanto aplicaremos uma transformada de Fourier dos dois lados da equação de autovalores. A transformada de Fourier sobre as componentes das funções envelope não causam problemas:

$$
F_{\nu}(\mathbf{x})=\sum_{\mathbf{k}} \frac{1}{\sqrt{\Omega}} e^{i \mathbf{k} \cdot \mathbf{x}} F_{\nu}(\mathbf{k})
$$

Devemos tomar um cuidado maior com a transformada no termo do potencial perturbativo. Se o potencial for suave o elemento de matriz $\left(\mathbf{k}|U| \mathbf{k}^{\prime}\right)$ será aproximadamente constante e poderemos escrever:

$$
U(\mathbf{x}) F_{\nu}(\mathbf{x})=\sum_{\mathbf{k}} \sum_{\mathbf{k}^{\prime}} \frac{1}{\sqrt{\Omega}} e^{i \mathbf{k} \cdot \mathbf{x}}\left(\mathbf{k}|U| \mathbf{k}^{\prime}\right) F_{\nu}\left(\mathbf{k}^{\prime}\right)
$$

Dessa forma a equação de autovalores no espaço direto será:

$$
E_{\nu} F_{\nu}(\mathbf{x})=\left[E_{\nu}^{n . p .}(-i \nabla)+U(\mathbf{x})\right] F_{\nu}(\mathbf{x})
$$

\subsection{Teoria de perturbações - Bandas não- degeneradas}

Nesta e na próxima seções iremos deduzir as expressões para as equações de massa efetiva e as funções envelope das diversas bandas. Nesta seção trataremos o caso de bandas não-degeneradas e na próxima o caso de bandas degeneradas. 
O ponto de mais alta simetria em sistemas baseados em GaAs é o ponto $\Gamma$. Estes são também, em sua maioria, materiais com 'gap' direto nesse mesmo ponto. Todos os sistemas a serem estudados neste trabalho mantêm estas características, então para simplificar a notação passaremos a partir de agora a fazer a substituição $\mathbf{k}_{0}=\mathbf{0}$.

\subsubsection{Hamiltoniana não-perturbada}

A equação de autovalores de um sistema sujeito apenas a um potencial cristalino é:

$$
\begin{aligned}
\left(\nu \mathbf{k} 0|H| \nu^{\prime} \mathbf{k}^{\prime} \mathbf{0}\right) & =\left(\nu \mathbf{0 0}\left|H_{\mathbf{k} \cdot \mathbf{p}}(\mathbf{k})\right| \nu^{\prime} \mathbf{0 0}\right)= \\
& =\left(\nu 00\left|H_{0}(\mathbf{k})+\frac{\hbar}{m_{0}} \mathbf{k} \cdot \mathbf{p}\right| \nu^{\prime} \mathbf{0 0}\right)
\end{aligned}
$$

Como definido anteriormente, temos:

$$
H_{0}(\mathbf{k})=\frac{\mathbf{p}^{2}}{2 m_{0}}+V(\mathbf{x})+\frac{\hbar^{2} \mathbf{k}^{2}}{2 m_{0}}
$$

onde a energia do Hamiltoniano não-perturbado é:

$$
\left(\nu \mathbf{0 0}\left|H_{0}(\mathbf{k})\right| \nu^{\prime} \mathbf{0 0}\right)=E_{\nu}^{0}(-i \nabla) \delta_{\nu \nu^{\prime}} \delta_{\mathbf{k k}^{\prime}} .
$$

Tomando o termo de $\mathbf{k} \cdot \mathbf{p}$ como perturbação ao sistema e desenvolvendo, em teoria de perturbações de segunda ordem, podemos escrever a equação de autovalores como:

$$
\begin{aligned}
E_{\nu}^{2}(\mathbf{k})=\left(\nu 00\left|H_{0}\right| \nu 00\right) & +\frac{\hbar \mathbf{k}}{m_{0}} \cdot(\nu 00|\mathbf{p}| \nu 00)+ \\
& +\frac{\hbar^{2}}{m_{0}^{2}} \sum_{\nu \neq \nu^{\prime}} \frac{\left|\left(\nu 00|\mathbf{k} \cdot \mathbf{p}| \nu^{\prime} \mathbf{0 0}\right)\right|^{2}}{E_{\nu}(\mathbf{0})-E_{\nu^{\prime}}(\mathbf{0})}
\end{aligned}
$$

Podemos também escrever os autovetores deste sistema:

$$
\left.\left.\mid \nu \mathrm{k} 0)^{1}=\mid \nu \mathrm{k} 0\right)+\frac{\hbar}{m_{0}} \sum_{\mu \neq \nu} \frac{(\mu 00|\mathbf{k} \cdot \mathbf{p}| \nu 00)}{E_{\mu}(0)-E_{\nu}(0)} \mid \mu \mathbf{k} 0\right) .
$$


No ponto $\Gamma$ temos, em nossos sistemas, ou um mínimo da banda de condução ou um máximo da banda de valência. O termo linear na equação (2.28), tem portanto, valor nulo:

$$
(\nu 00|\mathbf{p}| \nu \mathbf{0 0})=0
$$

Também para simplificar as expressões , pode-se redefinir a energia da banda no ponto $\Gamma$ como sendo o zero de energia do sistema:

$$
\left(\nu 00\left|H_{0}(\mathbf{0})\right| \nu 00\right)=E_{\nu}^{0}(\mathbf{0})=0 .
$$

Desta forma a equação de autovalores torna-se:

$$
E_{\nu}^{2}(\mathbf{k})=\frac{\hbar^{2}}{2 m_{0}}\left[\mathbf{k}^{2}+\frac{2}{m_{0}} \sum_{\alpha \beta} \sum_{\nu \neq \nu^{\prime}} \frac{\left(\nu 00\left|k_{\alpha} p_{\alpha}\right| \nu^{\prime} 00\right)\left(\nu^{\prime} 00\left|k_{\beta} p_{\beta}\right| \nu 00\right)}{E_{\nu}(\mathbf{0})-E_{\nu^{\prime}}(\mathbf{0})}\right] .
$$

Esta mesma equação pode ser reescrita, em uma forma mais simples, como uma equação de massa efetiva:

$$
E_{\nu}^{n . p .}=E_{\nu}^{2}(\mathbf{k})=\frac{\hbar^{2}}{2} \sum_{\alpha \beta} \mathrm{M}_{\nu \alpha \beta}^{-1} k_{\alpha} k_{\beta},
$$

onde definimos o tensor de massa efetiva como:

$$
\begin{aligned}
\mathbf{M}_{\nu \alpha \beta}=\left[\frac{\delta_{\alpha \beta}}{m_{0}}+\right. & \frac{1}{m_{0}^{2}} \sum_{\nu \neq \nu^{\prime}} \frac{\left(\nu 00\left|p_{\alpha}\right| \nu^{\prime} 00\right)\left(\nu^{\prime} 00\left|p_{\beta}\right| \nu 00\right)}{E_{\nu}(0)-E_{\nu^{\prime}}(0)}+ \\
& \left.+\frac{1}{m_{0}^{2}} \sum_{\nu \neq \nu^{\prime}} \frac{\left(\nu 00\left|p_{\beta}\right| \nu^{\prime} 00\right)\left(\nu^{\prime} 00\left|p_{\alpha}\right| \nu 00\right)}{E_{\nu}(0)-E_{\nu^{\prime}}(0)}\right]^{-1}
\end{aligned}
$$

\subsubsection{Hamiltoniana perturbada e funções envelope}

Vamos tratar agora a perturbação periódica ao potencial cristalino. Este sistema é descrito pelo Hamiltoniano:

$$
E_{\nu}{ }^{1}(\nu \mathbf{k} \mathbf{0} \mid \Psi)=\sum_{\nu^{\prime} \mathbf{k}^{\prime}}\left[{ }^{1}\left(\nu \mathbf{k} \mathbf{0}|H| \nu^{\prime} \mathbf{k}^{\prime} \mathbf{0}\right)^{1}+{ }^{1}\left(\nu \mathbf{k} \mathbf{0}|U| \nu^{\prime} \mathbf{k}^{\prime} \mathbf{0}\right)^{1}\right]^{1}\left(\nu^{\prime} \mathbf{k}^{\prime} \mathbf{0} \mid \Psi\right)
$$


As componentes da função envelope da banda $\nu_{0}$ no espaço recíproco são dados por:

$$
{ }^{1}(\nu \mathbf{k} 0 \mid \Psi)=F_{\nu}(\mathbf{k}) \delta_{\nu \nu^{\prime}}
$$

e os autovalores dessas funções são dados por:

$$
{ }^{1}\left(\nu \mathbf{k} 0|H| \nu^{\prime} \mathbf{k}^{\prime} \mathbf{0}\right)^{1}=E_{\nu}^{n . p .}(k) \delta_{\nu \nu^{\prime}} \delta_{\mathbf{k k}^{\prime}} .
$$

Substituindo $\left.\mid \nu^{\prime} \mathbf{k}^{\prime} \mathbf{0}\right)^{1}$ pela expressão determinada na seção anterior temos a seguinte expressão para o termo de perturbação:

$$
\begin{aligned}
{ }^{1}\left(\nu \mathbf{k} 0|U| \nu^{\prime} \mathbf{k}^{\prime} \mathbf{0}\right)^{1} & =\left(\nu \mathbf{k} \mathbf{0}|U| \nu^{\prime} \mathbf{k}^{\prime} \mathbf{0}\right)+ \\
& +\frac{\hbar}{m_{0}} \sum_{\mu \neq \nu^{\prime}} \frac{\left(\nu \mathbf{k} \mathbf{0}|U| \mu \mathbf{k}^{\prime} \mathbf{0}\right)\left(\mu \mathbf{0 0}\left|\mathbf{k}^{\prime} \cdot \mathbf{p}\right| \nu \mathbf{0 0}\right)}{E_{\mu}-E_{\nu^{\prime}}}+ \\
& +\frac{\hbar}{m_{0}} \sum_{\mu \neq \nu} \frac{\left(\mu \mathbf{k} \mathbf{0}|U| \nu^{\prime} \mathbf{k}^{\prime} \mathbf{0}\right)(\nu \mathbf{0 0}|\mathbf{k} \cdot \mathbf{p}| \mu \mathbf{0 0})}{E_{\mu}-E_{\nu}}
\end{aligned}
$$

que podemos reescrever como:

$$
\begin{aligned}
{ }^{1}\left(\nu \mathbf{k} 0|U| \nu^{\prime} \mathbf{k}^{\prime} \mathbf{0}\right)^{1} & =\left(\nu \mathbf{k} \mathbf{0}|U| \nu^{\prime} \mathbf{k}^{\prime} \mathbf{0}\right)+ \\
& +\frac{\hbar}{m_{0}}\left(\mathbf{k}|U| \mathbf{k}^{\prime}\right)\left[\sum_{\mu \neq \nu^{\prime}} \frac{\left(\nu \mathbf{0 0}\left|\left(\mathbf{k}-\mathbf{k}^{\prime}\right) \cdot \mathbf{p}\right| \nu^{\prime} \mathbf{0 0}\right)}{E_{\nu}-E_{\nu^{\prime}}}\right] .
\end{aligned}
$$

Podemos ainda simplificar extraindo o termo $\left(\mathbf{k}-\mathbf{k}^{\prime}\right)$ de dentro da soma:

$$
\begin{aligned}
{ }^{1}\left(\nu \mathbf{k} \mathbf{0}|U| \nu^{\prime} \mathbf{k}^{\prime} \mathbf{0}\right)^{1} & =\left(\mathbf{k}|U| \mathbf{k}^{\prime}\right) \delta_{\nu \nu^{\prime}}+ \\
& +\frac{\hbar}{m_{0}}\left(\mathbf{k}-\mathbf{k}^{\prime}\right)\left(\mathbf{k}|U| \mathbf{k}^{\prime}\right) \cdot\left[\sum_{\mu \neq \nu^{\prime}} \frac{\left(\nu \mathbf{0 0}|\mathbf{p}| \nu^{\prime} \mathbf{0 0}\right)}{E_{\nu}-E_{\nu^{\prime}}}\right] .
\end{aligned}
$$

Por fim, identifica-se o termo $\left(\mathbf{k}-\mathbf{k}^{\prime}\right)\left(\mathbf{k}|U| \mathbf{k}^{\prime}\right)$ com a derivada de $U$ :

$$
\begin{aligned}
{ }^{1}\left(\nu \mathbf{k} 0|U| \nu^{\prime} \mathbf{k}^{\prime} \mathbf{0}\right)^{1} & =\left(\mathbf{k}|U| \mathbf{k}^{\prime}\right) \delta_{\nu \nu^{\prime}}- \\
& -i \frac{\hbar}{m_{0}}\left(\mathbf{k}|\nabla U| \mathbf{k}^{\prime}\right) \cdot\left[\sum_{\mu \neq \nu^{\prime}} \frac{\left(\nu 00|\mathbf{p}| \nu^{\prime} \mathbf{0 0}\right)}{E_{\nu}-E_{\nu^{\prime}}}\right] .
\end{aligned}
$$


Sendo $U$ uma função suave, o segundo termo da expressão anterior pode ser desprezado, por depender da derivada de $U$. O termo perturbativo é, portanto, escrito como:

$$
{ }^{1}\left(\nu \mathbf{k} \mathbf{0}|U| \nu^{\prime} \mathbf{k}^{\prime} \mathbf{0}\right)^{1}=\left(\mathbf{k}|U| \mathbf{k}^{\prime}\right) \delta_{\nu \nu^{\prime}}
$$

Voltando à equação de autovalores no espaço recíproco:

$$
E_{\nu} F_{\nu}(\mathbf{k})=E_{\nu}^{n . p .} F_{\nu}(\mathbf{k})+\sum_{\mathbf{k}^{\prime}}\left(\mathbf{k}|U| \mathbf{k}^{\prime}\right) F_{\nu}\left(\mathbf{k}^{\prime}\right)
$$

Somando-se sobre todos os pontos do espaço recíproco:

$$
\begin{aligned}
E_{\nu} \sum_{\mathbf{k}} \frac{1}{\sqrt{\Omega}} e^{i \mathbf{k} \cdot \mathbf{x}} F_{\nu}(\mathbf{k}) & =\frac{\hbar^{2}}{2} \sum_{\mathbf{k}} \sum_{\alpha \beta} \mathbf{M}_{\nu_{0} \alpha \beta}^{-1} k_{\alpha} k_{\beta} \frac{1}{\sqrt{\Omega}} e^{i \mathbf{k} \cdot \mathbf{x}} F_{\nu}(\mathbf{k})+ \\
& +\sum_{\mathbf{k}} \sum_{\mathbf{k}^{\prime}}\left(\mathbf{k}|U| \mathbf{k}^{\prime}\right) \frac{1}{\sqrt{\Omega}} e^{i \mathbf{k} \cdot \mathbf{x}} F_{\nu}\left(\mathbf{k}^{\prime}\right)
\end{aligned}
$$

podemos obter a equação de autovalores para o espaço direto:

$$
\left[-\frac{\hbar^{2}}{2} \sum_{\alpha \beta} \mathbf{M}_{\nu_{0} \alpha \beta}^{-1} \frac{\partial}{\partial_{x_{\alpha}}} \frac{\partial}{\partial_{x_{\beta}}}+U(\mathbf{x})\right] F_{\nu}(\mathbf{x})=E_{\nu} F_{\nu}(\mathbf{x})
$$

\subsection{Teoria de perturbações - Bandas de- generadas}

No caso de bandas degeneradas é necessária a introdução de novos índices. Para indicar a banda utilizar-se-á o índice $\lambda$ e para os momentos angulares das sub-bandas o índice $\mu$. 


\subsubsection{Hamiltoniana não-perturbada}

Usando teoria de perturbações para sistemas degenerados, temos a seguinte expressão para os autovetores de nosso sistema:

$$
\begin{aligned}
\mid \lambda \mu \mathbf{k} 0)^{1} & \left.=\mid \lambda \mu \mathbf{k} \mathbf{0})+\frac{\hbar}{m_{0}} \sum_{\mu^{\prime}} \sum_{\lambda^{\prime} \neq \lambda} \frac{\left(\lambda^{\prime} \mu^{\prime} \mathbf{0 0}|\mathbf{k} \cdot \mathbf{p}| \lambda \mu \mathbf{0 0}\right)}{E_{\lambda^{\prime}}(\mathbf{0})-E_{\lambda}(\mathbf{0})} \mid \lambda^{\prime} \mu^{\prime} \mathbf{k} \mathbf{0}\right)- \\
& \left.-\frac{1}{2} \frac{\hbar^{2}}{m_{0}^{2}} \sum_{\mu^{\prime}} \sum_{\lambda^{\prime} \neq \lambda} \frac{\left|\left(\lambda^{\prime} \mu^{\prime} \mathbf{0 0}|\mathbf{k} \cdot \mathbf{p}| \lambda \mu \mathbf{0 0}\right)\right|^{2}}{\left(E_{\lambda^{\prime}}(\mathbf{0})-E_{\lambda}(\mathbf{0})\right)^{2}} \mid \lambda^{\prime} \mu^{\prime} \mathbf{k} \mathbf{0}\right)
\end{aligned}
$$

Da mesma maneira que na seção anterior, podemos reescrever a equação de autovalores na base das funções de Bloch:

$$
{ }^{1}\left(\lambda \mu \mathbf{k 0} 0|H| \lambda^{\prime} \mu^{\prime} \mathbf{k}^{\prime} \mathbf{0}\right)^{1}={ }^{1}\left(\lambda \mu \mathbf{0 0}\left|H_{\mathbf{k} \cdot \mathbf{p}}(\mathbf{k})\right| \lambda \mu^{\prime} \mathbf{0 0}\right)^{1} \delta_{\mathbf{k k}^{\prime}} \delta_{\lambda \lambda^{\prime}}
$$

Seguindo ainda os passos da seção anterior, podemos escrever a equação da massa efetiva para este sistema não-perturbado:

$$
{ }^{1}\left(\lambda \mu \mathbf{k} 0|H| \lambda^{\prime} \mu^{\prime} \mathbf{k}^{\prime} \mathbf{0}\right)^{1}=\sum_{\alpha \beta} \mathbf{D}_{\mu \mu^{\prime}}^{\alpha \beta} k_{\alpha} k_{\beta} \delta_{\mathbf{k} \mathbf{k}^{\prime}} \delta_{\lambda \lambda^{\prime}}
$$

onde $D_{\mu \mu^{\prime}}^{\alpha \beta}$ é o tensor inverso da massa efetiva e é definido como:

$$
\mathbf{D}_{\mu \mu^{\prime}}^{\alpha \beta}=\frac{\hbar^{2}}{2 m_{0}}\left[\delta_{\alpha \beta} \delta_{\mu \mu^{\prime}}+\frac{1}{m_{0}}\left(\mu\left|p_{\alpha} \frac{1}{E_{v}-H} p_{\beta}+p_{\beta} \frac{1}{E_{v}-H} p_{\alpha}\right| \mu^{\prime}\right)\right]
$$

\subsubsection{Hamiltoniana perturbada e funções envelope}

Seguindo os passos da seção anterior, também para o Hamiltoniano perturbado, pode-se ver que o potencial perturbativo pode ser descrito por:

$$
{ }^{1}\left(\lambda \mu \mathbf{k} \mathbf{0}|U| \lambda^{\prime} \mu^{\prime} \mathbf{k}^{\prime} \mathbf{0}\right)^{1}=\left(\mathbf{k}|U| \mathbf{k}^{\prime}\right) \delta_{\lambda \lambda^{\prime}} \delta_{\mu \mu^{\prime}}
$$


Desta maneira a equação de autovalores tem a forma:

$E_{\lambda \mu}{ }^{1}(\lambda \mu \mathbf{k} 0 \mid \Psi)=\sum_{\mu^{\prime} \mathbf{k}^{\prime}}\left[-\sum_{\alpha \beta} \mathbf{D}_{\mu \mu^{\prime}}^{\alpha \beta} k_{\alpha} k_{\beta} \delta_{k k^{\prime}}+\left(\mathbf{k}|U| \mathbf{k}^{\prime}\right) \delta_{\mu \mu^{\prime}}\right]^{1}\left(\lambda \mu^{\prime} \mathbf{k}^{\prime} 0 \mid \Psi\right)$

e a função envelope pode ser definida como:

$$
{ }^{1}(\lambda \mu \mathbf{k} 0 \mid \Psi)=F_{\lambda \mu}(\mathbf{k}) \text {. }
$$

A função envelope de uma banda no espaço direto tem múltiplos componentes e pode ser escrita como uma soma:

$$
E_{\lambda \mu} F_{\lambda \mu}(\mathbf{x})=\sum_{\mu^{\prime}}\left[-\sum_{\alpha \beta} \frac{\partial}{\partial_{x_{\alpha}}} \mathbf{D}_{\mu \mu^{\prime}}^{\alpha \beta} \frac{\partial}{\partial_{x_{\beta}}}+U(\mathbf{x}) \delta_{\mu \mu^{\prime}}\right] F_{\lambda \mu^{\prime}}(\mathbf{x})
$$

ou como uma equação tensorial:

$$
[-(\nabla|\mathcal{D}| \nabla)+U(\mathbf{x}) \mathbf{1}] F(\mathbf{x})=E F(\mathbf{x})
$$

\subsection{Os Hamiltonianos $\mathrm{k} \cdot \mathrm{p}$}

Com a equação da massa efetiva deduzida, passaremos à formulação dos Hamiltonianos k · p. Três diferentes Hamiltonianos serão aqui descritos:

1. o Hamiltoniano $6 \times 6$ que descreve a banda de valência, englobando buracos leves, pesados e 'split-off', proposto por Luttinger e $\mathrm{Kohn}^{[36]}$.

2. o Hamiltoniano $4 \times 4$ que simplifica a banda de valência, tratando apenas de buracos leves e pesados, proposto também por Luttinger e Kohn no mesmo artigo ${ }^{[36]}$.

3. o proposto por Kane $(8 \times 8)^{[57]}$ que além de levar em conta a banda de valência de maneira muito semelhante à aproximação anterior, leva em conta a banda de condução. 


\subsubsection{Matrizes de Luttinger-Kohn}

Levando-se em conta apenas as partes espaciais das funções de onda desse sistema, o Hamiltoniano pode ser escrito como:

$$
\left(\begin{array}{lll}
E_{v}^{\prime}+L k_{x}^{2}+M\left(k_{y}^{2}+k_{z}^{2}\right) & N k_{x} k_{y} & N k_{x} k_{z} \\
N k_{x} k_{y} & E_{v}^{\prime}+L k_{x}^{2}+M\left(k_{y}^{2}+k_{z}^{2}\right) & N k_{y} k_{z} \\
N k_{x} k_{z} & N k_{y} k_{z} & E_{v}^{\prime}+L k_{x}^{2}+M\left(k_{y}^{2}+k_{z}^{2}\right)
\end{array}\right),
$$

onde os vetores de onda seguem a ordem $\mid x),(y)$ e $\mid z)$,

$$
E_{v}^{\prime}=E_{v}+\frac{\hbar^{2} k^{2}}{2 m_{0}},
$$

e :

$$
\begin{aligned}
L & =\mathbf{D}_{x x}^{x x}, \\
M & =\mathbf{D}_{x x}^{y y}, \\
N & =\mathbf{D}_{x y}^{x y}+\mathbf{D}_{x y}^{y x} .
\end{aligned}
$$

Esses parâmetros podem também ser determinados através dos chamados parâmetros de Luttinger-Kohn, $\gamma_{1}, \gamma_{2}$ e $\gamma_{3}$. Tais parâmetros são determinados experimentalmente e se relacionam com os termos da matriz através das relações seguintes:

$$
\begin{aligned}
L & =\gamma_{1}+4 \gamma_{2} \\
M & =\gamma_{1}-2 \gamma_{2}, \\
N & =6 \gamma_{3} .
\end{aligned}
$$

Ao introduzirmos o spin nesse cálculo (sem levar em conta ainda a interação spin-órbita), vemos que cada um desses estados é bidegenerado. Então, a matriz completa para a banda de valência pode ser escrita como:

$$
H_{\mathbf{k} \cdot \mathbf{p}}=\left(\begin{array}{rr}
H_{0} & 0 \\
0 & H_{0}
\end{array}\right)
$$


onde o primeiro bloco representa os estados 'spin up' $(\uparrow)$ e o segundo os estados 'spin down' $(\downarrow)$.

Para descrever corretamente o nosso sistema devemos agora introduzir a interação spin-órbita em nosso cálculo. Faremos isto incorporando um termo para essa interação ao nosso Hamiltoniano. Este novo termo é descrito pela seguinte expressão:

$$
H_{s o}=\frac{\hbar}{2 m_{0}^{2} c^{2}}(\nabla \wedge \mathbf{p}) \cdot \sigma
$$

onde $\sigma=\sigma_{x} \mathbf{i}+\sigma_{y} \mathbf{j}+\sigma_{z} \mathbf{k}$ e $\sigma_{i}$ são as matrizes de Pauli. Ao explicitarmos os termos desta matriz, usando a mesma base utilizada anteriormente, escrevemos esta matriz como:

$$
H_{s o}=\frac{\Delta}{3}\left(\begin{array}{rrrrrr}
0 & -i & 0 & 0 & 0 & 1 \\
i & 0 & 0 & 0 & 0 & -i \\
0 & 0 & 0 & -1 & i & 0 \\
0 & 0 & -1 & 0 & i & 0 \\
0 & 0 & -i & -i & 0 & 0 \\
1 & i & 0 & 0 & 0 & 0 .
\end{array}\right)
$$

onde $\Delta$ é o desdobramento entre as minibandas de buracos leves e pesados e a minibanda com os estados de 'split-off'.

Esta matriz é diagonalizada pela seguinte transformação unitária:

$$
U=\left(\begin{array}{rrrrrr}
\frac{1}{\sqrt{2}} & 0 & \frac{1}{\sqrt{6}} & 0 & 0 & \frac{-i}{\sqrt{3}} \\
\frac{i}{\sqrt{2}} & 0 & \frac{-i}{\sqrt{6}} & 0 & 0 & \frac{-1}{\sqrt{3}} \\
0 & \frac{-2 i}{\sqrt{6}} & 0 & 0 & \frac{1}{\sqrt{3}} & 0 \\
0 & \frac{i}{\sqrt{6}} & 0 & \frac{i}{\sqrt{2}} & \frac{1}{\sqrt{3}} & 0 \\
0 & \frac{-1}{\sqrt{6}} & 0 & \frac{1}{\sqrt{2}} & \frac{i}{\sqrt{3}} & 0 \\
0 & 0 & \frac{2}{\sqrt{6}} & 0 & 0 & \frac{i}{\sqrt{3}}
\end{array}\right) .
$$

Apenas dois autovalores são obtidos. O primeiro, quadridegenerado:

$$
\left.\left.\mid E_{1}\right)=\mid E_{2}\right)=\left|E_{3}\right|=\left|E_{4}\right|=\frac{\Delta}{3},
$$


com os seguintes autovetores:

$$
\begin{aligned}
\left.\mid E_{1}\right) & =\frac{1}{\sqrt{2}}(1, i, 0,0,0,0), \\
\left.\mid E_{2}\right) & =\frac{i}{\sqrt{6}}(0,0,-2,1, i, 0), \\
\left.\mid E_{3}\right) & =\frac{1}{\sqrt{6}}(1,-i, 0,0,0,2), \\
\left.\mid E_{4}\right) & =\frac{i}{\sqrt{2}}(0,0,0,1,-i, 0),
\end{aligned}
$$

e o segundo, bidegenerado:

$$
\left.\left.\mid E_{5}\right)=\mid E_{6}\right)=-\frac{2 \Delta}{3},
$$

com os seguintes autovetores:

$$
\begin{aligned}
& \left.\mid E_{1}\right)=\frac{1}{\sqrt{3}}(0,0,1,1, i, 0), \\
& \left.\mid E_{2}\right)=\frac{i}{\sqrt{3}}(-1, i, 0,0,0,1) .
\end{aligned}
$$

Pode-se reescrever essa base valendo-se da notação de momento angular total, $j, m_{j}$, ou também, explicitando-se as funções de onda espaciais e os termos de spin:

$$
\begin{aligned}
&\left.\mid \frac{3}{2}, \frac{3}{2}\right)\left.\left.=\mid E_{1}\right)=\frac{1}{\sqrt{2}} \mid(X+i Y) \uparrow\right), \\
&\left.\mid \frac{3}{2}, \frac{1}{2}\right)\left.\left.\left.=\mid E_{2}\right)=\frac{i}{\sqrt{6}}[-2 \mid Z \uparrow)+\mid(X+i Y) \downarrow\right)\right], \\
&\left.\mid \frac{3}{2},-\frac{1}{2}\right)\left.\left.\left.=\mid E_{3}\right)=\frac{1}{\sqrt{6}}[2 \mid Z \downarrow)+\mid(X-i Y) \uparrow\right)\right], \\
&\left.\left.\left|\frac{3}{2},-\frac{3}{2}\right\rangle=\mid E_{4}\right)=\frac{i}{\sqrt{2}} \mid(X-i Y) \downarrow\right), \\
&\left.\left.\left|\frac{1}{2}, \frac{1}{2}\right\rangle=\mid E_{1}\right)=\frac{1}{\sqrt{3}}[|Z \uparrow\rangle+\mid(X+i Y) \downarrow)\right], \\
&\left.\left.\left.\left|\frac{1}{2},-\frac{1}{2}\right\rangle=\mid E_{2}\right)=\frac{i}{\sqrt{3}}[\mid Z \downarrow) \mid(X-i Y) \uparrow\right)\right] .
\end{aligned}
$$


Aplicando-se a mesma transformação unitária $U$ sobre a matriz $H_{\mathbf{k} \cdot \mathbf{p}}$ anteriormente obtida, encontramos:

$$
H_{\mathbf{k} \cdot \mathbf{p}}=\left(\begin{array}{cccccc}
Q & S & R & 0 & \frac{i}{\sqrt{2}} S & -i \sqrt{2} R \\
S^{*} & T & 0 & R & \frac{-i}{\sqrt{2}}(Q-T) & i \sqrt{\frac{3}{2}} S \\
R^{*} & 0 & T & -S & -i \sqrt{\frac{3}{2}} S^{*} & \frac{-i}{\sqrt{2}}(Q-T) \\
0 & R^{*} & -S^{*} & Q & -i \sqrt{2} R^{*} & \frac{-i}{\sqrt{2}} S^{*} \\
\frac{-i}{\sqrt{2}} S^{*} & \frac{i}{\sqrt{2}}(Q-T) & i \sqrt{\frac{3}{2}} S & i \sqrt{2} R & \frac{1}{2}(Q+T) & 0 \\
i \sqrt{2} R^{*} & -i \sqrt{\frac{3}{2}} S^{*} & \frac{i}{\sqrt{2}}(Q-T) & \frac{i}{\sqrt{2}} S & 0 & \frac{1}{2}(Q+T)
\end{array}\right) .
$$

A Hamiltoniana $H_{\mathbf{k} \cdot \pi}$, onde a substituição de $\mathbf{p}$ por $\pi$ significa que incluiu-se o termo de spin-órbita, é então definida pela equação:

$$
H_{\mathbf{k} \cdot \pi}=H_{\mathbf{k} \cdot \mathbf{p}}+H_{s o}-\frac{1}{3} \Delta 1
$$

onde 1 é uma matriz unidade. $\mathrm{O}$ termo $-\frac{1}{3} \Delta 1$ foi introduzido para que o zero de energia do sistema ficasse no topo da banda de valência. A matriz de Luttinger-Kohn incluindo os estados de buracos leves, pesados e 'split-off' tem, então, a forma:

$$
H_{\mathbf{k} \cdot \pi}=\left(\begin{array}{cccccc}
Q & S & R & 0 & \frac{i}{\sqrt{2}} S & -i \sqrt{2} R \\
S^{*} & T & 0 & R & \frac{-i}{\sqrt{2}}(Q-T) & i \sqrt{\frac{3}{2}} S \\
R^{*} & 0 & T & -S & -i \sqrt{\frac{3}{2}} S^{*} & \frac{-i}{\sqrt{2}}(Q-T) \\
0 & R^{*} & -S^{*} & Q & -i \sqrt{2} R^{*} & \frac{-i}{\sqrt{2}} S^{*} \\
\frac{-i}{\sqrt{2}} S^{*} & \frac{i}{\sqrt{2}}(Q-T) & i \sqrt{\frac{3}{2}} S & i \sqrt{2} R & \frac{1}{2}(Q+T)-\Delta & 0 \\
i \sqrt{2} R^{*} & -i \sqrt{\frac{3}{2}} S^{*} & \frac{i}{\sqrt{2}}(Q-T) & \frac{i}{\sqrt{2}} S & 0 & \frac{1}{2}(Q+T)-\Delta
\end{array}\right),
$$

onde:

$$
\begin{gathered}
Q=-\frac{\hbar^{2}}{2 m_{0}}\left[\left(\gamma_{1}+\gamma_{2}\right)\left(\mathbf{k}_{x}^{2}+\mathbf{k}_{y}^{2}\right)+\left(\gamma_{1}-2 \gamma_{2}\right) \mathbf{k}_{z}^{2}\right] \\
T=-\frac{\hbar^{2}}{2 m_{0}}\left[\left(\gamma_{1}-\gamma_{2}\right)\left(\mathbf{k}_{x}^{2}+\mathbf{k}_{y}^{2}\right)+\left(\gamma_{1}+2 \gamma_{2}\right) \mathbf{k}_{z}^{2}\right] \\
S=i \frac{\hbar^{2}}{m_{0}} \sqrt{3} \gamma_{3}\left(\mathbf{k}_{x}-i \mathbf{k}_{y}\right) \mathbf{k}_{z}
\end{gathered}
$$




$$
R=-\frac{\hbar^{2}}{2 m_{0}} \sqrt{3}\left[\gamma_{2}\left(\mathbf{k}_{x}^{2}-\mathbf{k}_{y}^{2}\right)-2 i \gamma_{3} \mathbf{k}_{x} \mathbf{k}_{y}\right]
$$

Podemos reescrever esses termos usando unidades Rydberg $(\hbar=c=1$, $m_{0}=\frac{1}{2}$ e $e=\sqrt{2}$ ):

$$
\begin{gathered}
Q=-\left(\gamma_{1}+\gamma_{2}\right)\left(\mathbf{k}_{x}^{2}+\mathbf{k}_{y}^{2}\right)+\left(2 \gamma_{2}-\gamma_{1}\right) \mathbf{k}_{z}^{2} \\
T=-\left(\gamma_{1}-\gamma_{2}\right)\left(\mathbf{k}_{x}^{2}+\mathbf{k}_{y}^{2}\right)-\left(2 \gamma_{2}+\gamma_{1}\right) \mathbf{k}_{z}^{2} \\
S=i 2 \sqrt{3} \gamma_{3}\left(\mathbf{k}_{x}-i \mathbf{k}_{y}\right) \mathbf{k}_{z} \\
R=-\sqrt{3}\left[\gamma_{2}\left(\mathbf{k}_{x}^{2}-\mathbf{k}_{y}^{2}\right)-2 i \gamma_{3} \mathbf{k}_{x} \mathbf{k}_{y}\right] .
\end{gathered}
$$

Observando-se os termos de $\left(j, m_{j}\left|H_{\text {k.p }}\right| j^{\prime}, m_{j^{\prime}}\right)$ com $j \neq j^{\prime}$, podemos determinar uma dependência deles com as expressões $(L-M) \mathbf{k}^{2} \mathbf{e}$ $N \mathbf{k}^{2}$. Então, se os valores desses termos forem muito menores que $\Delta$, podem ser desprezados. Para o GaAs, em pontos $\mathbf{k}$ próximos a $\Gamma$ essa relação é verdadeira. Dessa maneira, $H_{\mathbf{k} . \mathbf{p}}$ pode ser blocodiagonalizado levando a duas matrizes não-interagentes, uma, de dimensão $4 \times 4$, que descreve os estados de buracos leves e pesados (i.e., $j=\frac{3}{2}$ ):

$$
H_{\text {k.p }}\left(j=\frac{3}{2}\right)=\left(\begin{array}{cccc}
Q & S & R & 0 \\
S^{*} & T & 0 & R \\
R^{*} & 0 & T & -S \\
0 & R^{*} & -S^{*} & Q
\end{array}\right) \text {, }
$$

e outra, de dimensão $2 \times 2$, que descreve os estados 'split-off' $\left(j=\frac{1}{2}\right)$ :

$$
H_{\text {k.p }}\left(j=\frac{1}{2}\right)=\left(\begin{array}{cc}
\frac{1}{2}(Q+T)-\Delta & 0 \\
0 & \frac{1}{2}(Q+T)-\Delta
\end{array}\right) .
$$

\subsubsection{Matriz de Kane}

Para se determinar a matriz de Kane deve-se seguir um procedimento semelhante ao que adotamos até aqui. A diferença entre o procedimento 
adotado para a obtenção dos Hamiltonianos de Luttinger-Kohn e de Kane reside no fato que, no primeiro os estados tratados exatamente são apenas os estados da banda de valência e no último, além destes são incluídos os estados da banda de condução. Seguindo esse espírito, escrevemos o Hamiltoniano da parte espacial das funções de onda da seguinte maneira:

$$
\left(\begin{array}{cccc}
A k^{2}+E_{c}^{\prime} & i P k_{x} & i P k_{y} & i P k_{z} \\
-i P k_{x} & E_{v}^{\prime}+\bar{L} k_{x}^{2}+\bar{M}\left(k_{y}^{2}+k_{z}^{2}\right) & \bar{N} k_{x} k_{y} & \bar{N} k_{x} k_{z} \\
-i P k_{y} & \bar{N} k_{x} k_{y} & E_{v}^{\prime}+\bar{L} k_{y}^{2}+\bar{M}\left(k_{x}^{2}+k_{z}^{2}\right) & \bar{N} k_{y} k_{z} \\
-i P k_{z} & \bar{N} k_{x} k_{z} & \bar{N} k_{y} k_{z} & E_{v}^{\prime}+\bar{L} k_{z}^{2}+\bar{M}\left(k_{x}^{2}+k_{y}^{2}\right)
\end{array}\right) \text {, }
$$

onde:

$$
\begin{array}{r}
E_{c}^{\prime}=E_{c}+\frac{\hbar^{2} k^{2}}{2 m_{0}}, \\
E_{v}^{\prime}=E_{v}+\frac{\hbar^{2} k^{2}}{2 m_{0}}, \\
A=\frac{\hbar^{2}}{m_{e}},
\end{array}
$$

onde $m_{e}$ é a massa efetiva do elétron na banda de condução e $P$, o elemento de matriz de Kane, é o elemento da matriz de momentos entre estados das bandas de condução e valência:

$$
P=-i \frac{\hbar}{m_{0}}\left(x\left|p_{x}\right| u_{j}\right)
$$

As definições dos termos $\bar{L}, \bar{M}$ e $\bar{N}$ diferem das definições de Kohn e Luttinger $(L, M$ e $N)$ por não incluírem a interação entre os estados da banda de valência e os da banda de condução. Em virtude disso, 
esses parâmetros são definidos como:

$$
\begin{aligned}
\bar{L} & =\mathbf{D}_{x x}^{x x}+\frac{2 m_{0}}{\hbar^{2}} \frac{P^{2}}{E_{g}} \\
\bar{M} & =\mathbf{D}_{y y}^{x x}, \\
\bar{N} & =\mathbf{D}_{x y}^{x y}+\mathbf{D}_{x y}^{y x}+\frac{2 m_{0}}{\hbar^{2}} \frac{P^{2}}{E_{g}}
\end{aligned}
$$

Neste sistema a expressão do termo de spin-órbita é a seguinte matriz:

$$
H_{s o}=\frac{\Delta}{3}\left(\begin{array}{rrrrrrrr}
0 & 0 & 0 & 0 & 0 & 0 & 0 & 0 \\
0 & 0 & 0 & 0 & 0 & 0 & 0 & 0 \\
0 & 0 & 0 & -i & 0 & 0 & 0 & 1 \\
0 & 0 & i & 0 & 0 & 0 & 0 & -i \\
0 & 0 & 0 & 0 & 0 & -1 & i & 0 \\
0 & 0 & 0 & 0 & -1 & 0 & i & 0 \\
0 & 0 & 0 & 0 & -i & -i & 0 & 0 \\
0 & 0 & 1 & i & 0 & 0 & 0 & 0
\end{array}\right)
$$

A matriz unitária que diagonaliza $H_{\text {so }}$ é:

$$
u=\left(\begin{array}{rrrrrrrr}
1 & 0 & 0 & 0 & 0 & 0 & 0 & 0 \\
0 & 1 & 0 & 0 & 0 & 0 & 0 & 0 \\
0 & 0 & \frac{1}{\sqrt{2}} & 0 & \frac{1}{\sqrt{6}} & 0 & 0 & \frac{-i}{\sqrt{3}} \\
0 & 0 & \frac{i}{\sqrt{2}} & 0 & \frac{-i}{\sqrt{6}} & 0 & 0 & \frac{-1}{\sqrt{3}} \\
0 & 0 & 0 & \frac{-2 i}{\sqrt{6}} & 0 & 0 & \frac{1}{\sqrt{3}} & 0 \\
0 & 0 & 0 & \frac{i}{\sqrt{6}} & 0 & \frac{i}{\sqrt{2}} & \frac{1}{\sqrt{3}} & 0 \\
0 & 0 & 0 & \frac{-1}{\sqrt{6}} & 0 & \frac{1}{\sqrt{2}} & \frac{i}{\sqrt{3}} & 0 \\
0 & 0 & 0 & 0 & \frac{2}{\sqrt{6}} & 0 & 0 & \frac{i}{\sqrt{3}}
\end{array}\right) .
$$

Usando o mesmo procedimento que anteriormente, obtemos a expressão 
para o Hamiltoniano proposto por Kane:

$\left(\begin{array}{cccccccc}E_{g}+A k^{2} & 0 & i P_{+} & \sqrt{\frac{2}{3}} P_{z} & i \sqrt{\frac{1}{3}} P_{-} & 0 & i \sqrt{\frac{1}{3}} P_{z} & \sqrt{\frac{2}{3}} P_{-} \\ 0 & E_{g}+A k^{2} & 0 & -\sqrt{\frac{1}{3}} P_{+} & i \sqrt{\frac{2}{3}} P_{z} & -P_{-} & i \sqrt{\frac{2}{3}} P_{+} & -\sqrt{\frac{1}{3}} P_{z} \\ -i P_{-} & 0 & Q & S & R & 0 & \frac{i}{\sqrt{2}} S & -i \sqrt{2} R \\ \sqrt{\frac{2}{3}} P_{z} & -\sqrt{\frac{1}{3}} P_{-} & S^{*} & T & 0 & R & \frac{-i}{\sqrt{2}}(Q-T) & \dot{2} \frac{3}{2} S \\ -i \sqrt{\frac{1}{3}} P_{+} & -i \sqrt{\frac{2}{3}} P_{z} & R^{*} & 0 & T & -S & -i \sqrt{\frac{3}{2}} S^{*} & \frac{-i}{\sqrt{2}}(Q-T) \\ 0 & -P_{+} & 0 & R^{*} & -S^{*} & Q & -i \sqrt{2} R^{*} & \frac{-i}{\sqrt{2}} S^{*} \\ -i \sqrt{\frac{1}{3}} P_{z} & -i \sqrt{\frac{2}{3}} P_{-} & \frac{-i}{\sqrt{2}} S^{*} & \frac{i}{\sqrt{2}}(Q-T) & \dot{i} \sqrt{\frac{3}{2}} S & \dot{i} \sqrt{2} R & \frac{1}{2}(Q+T)-\Delta & 0 \\ \sqrt{\frac{2}{3}} P_{+} & -\sqrt{\frac{1}{3}} P_{z} & i \sqrt{2} R^{*} & -i \sqrt{\frac{3}{2}} S^{*} & \frac{i}{\sqrt{2}}(Q-T) & \frac{i}{\sqrt{2}} S & 0 & \frac{1}{2}(Q+T)-\Delta\end{array}\right)$,

onde $E_{g}, P_{+}, P_{-}$e $P_{z}$ são definidos como:

$$
\begin{aligned}
& E_{g}=E_{c}-E_{v}-\frac{\Delta}{3} \\
& P_{+}=P\left(k_{x}+i k_{y}\right) \\
& P_{-}=P\left(k_{x}-i k_{y}\right) \\
& P_{z}=P k_{z}
\end{aligned}
$$

e $Q, S, R$, e $T$ são definidos como anteriormente:

$$
\begin{array}{r}
Q=-\frac{\hbar^{2}}{2 m_{0}}\left[\left(\overline{\gamma_{1}}+\overline{\gamma_{2}}\right)\left(\mathbf{k}_{x}^{2}+\mathbf{k}_{y}^{2}\right)+\left(\overline{\gamma_{1}}-2 \overline{\gamma_{2}}\right) \mathbf{k}_{z}^{2}\right] \\
T=-\frac{\hbar^{2}}{2 m_{0}}\left[\left(\overline{\gamma_{1}}-\overline{\gamma_{2}}\right)\left(\mathbf{k}_{x}^{2}+\mathbf{k}_{y}^{2}\right)+\left(\overline{\gamma_{1}}+2 \overline{\gamma_{2}}\right) \mathbf{k}_{z}^{2}\right] \\
S=i \frac{\hbar^{2}}{m_{0}} \sqrt{3} \overline{\gamma_{3}}\left(\mathbf{k}_{x}-i \mathbf{k}_{y}\right) \mathbf{k}_{z}, \\
R=-\frac{\hbar^{2}}{2 m_{0}} \sqrt{3}\left[\overline{\gamma_{2}}\left(\mathbf{k}_{x}^{2}-\mathbf{k}_{y}^{2}\right)-2 i \overline{\gamma_{3}} \mathbf{k}_{x} \mathbf{k}_{y}\right] .
\end{array}
$$




\subsection{Determinação dos parâmetros de Kane através de massas efetivas experimentais}

Em cálculos de estrutura de bandas para elétrons e buracos em microestruturas semicondutoras, a teoria da massa efetiva é usada extensivamente. Nesta teoria, as estruturas de bandas de materiais volumétricos são representadas por Hamiltonianos $\mathbf{k} \cdot \mathbf{p}$. Dependendo da sofisticação do cálculo, usam-se Hamiltonianos $(1 \times 1)$ para buracos ou elétrons (modelos de uma banda), Hamiltonianos $(4 \times 4)$ ou $(6 \times 6)$ para buracos (modelo de Luttinger-Kohn) ou Hamiltonianos $(8 \times 8)$ para buracos e elétrons (modelo de Kane). Como vimos, no modelo de Kane além das interações $\mathbf{k} \cdot \mathbf{p}$ entre estados da banda de valência, as interações entre estados das bandas de condução e de valência são levadas em conta exatamente, enquanto as interaçōes com bandas remotas são tratadas via teoria de perturbações de segunda ordem. Por outro lado, no modelo de Luttinger-Kohn, somente as interações entre estados da banda de valência são levadas em conta de maneira exata, enquanto que as interações da banda de valência com as outras bandas, inclusive com a de condução são tratadas via teoria de perturbações em segunda ordem. Formalmente o modelo de Kane torna-se o modelo de Luttinger-Kohn se os elementos da matriz de Kane entre as bandas de condução e de valência forem nulos.

O modelo de Kane é necessário se considerarmos materiais com 'gap' direto pequeno no centro da primeira zona de Brillouin (ponto $\Gamma$ ). Para materiais com 'gap' direto maior, como é o caso de GaAs e AlAs, o modelo de Kane se aplica também de maneira boa, mas em muitos casos o modelo de Luttinger-Kohn é suficiente. No entanto, existem casos em que o modelo de Kane é recomendado mesmo para materiais com 'gap' maior. Isto ocorre, por exemplo, se queremos calcular as transições óticas entre os elementos de matriz de buracos e de elétrons. Caso em que tratar ambos os estados, buracos e elétrons, dentro do mesmo modelo e com o mesmo nível de precisão configura-se numa grande vantagem. 
A aplicação do modelo de Kane para tais materiais, leva à necessidade do cálculo dos parâmetros de Kane. Como os parâmetros de Luttinger do modelo de Luttinger-Kohn eles podem ser calculados através das massas efetivas de buracos e elétrons em diferentes direções do espaço recíproco. Tais cálculos foram feitos para $G a A s$ e $A l A s,(G a, A l) A s$, $C d T e,(C d, M n) T e,(G a, I n) A s$ e $(A l, I n) A s$ por Cohen e Marques ${ }^{[58]}$.

As relações para os parâmetros de Kane estão, no entanto, em desacordo entre as referências $[58,59] \mathrm{e},[60,61]$. Abaixo mostraremos que esta diferença é devida a um diferente entendimento dos parâmetros de Luttinger no modelo de Kane, e que na verdade se eqüivalem. Também mostraremos que o elemento de momento $\mathrm{P}$ da matriz de Kane, assim como os parâmetros de Luttinger calculados na referência [58] podem variar de maneira considerável se usarmos em seu cálculo conjuntos de massas efetivas ligeiramente diferentes. A razão para esta instabilidade inesperada é analisada e dessa análise derivamos condições que as massas efetivas devem cumprir para que os modelos de Kane ou de Luttinger-Kohn sejam aplicáveis.

\subsubsection{Definição dos parâmetros de Luttinger no modelo de Kane}

A partir das expressões (2.59) a (2.61) podemos determinar os parâmetros de Luttinger em termos das constantes $L, M$ e $N$. As expressões para esses são:

$$
\begin{aligned}
\gamma_{1} & =-\frac{2 m_{0}}{\hbar^{2}} \frac{1}{3}(L+2 M) \\
\gamma_{2} & =-\frac{2 m_{0}}{\hbar^{2}} \frac{1}{6}(L-M) \\
\gamma_{3} & =-\frac{2 m_{0}}{\hbar^{2}} \frac{1}{6} N
\end{aligned}
$$

Como já dissemos, os parâmetros de Luttinger $L, M$ e $N$ diferem de seus congêneres de Kane $\bar{L}, \bar{M}$ e $\bar{N}$ por incluírem em sua formulação as interações dos estados da banda de condução. Os parâmetros desses 
dois conjuntos se relacionam através das seguintes expressōes:

$$
\begin{aligned}
L & =\bar{L}-\frac{2 m_{0}}{\hbar^{2}} \frac{P^{2}}{E_{g}} \\
M & =\bar{M} \\
N & =\bar{N}-\frac{2 m_{0}}{\hbar^{2}} \frac{P^{2}}{E_{g}}
\end{aligned}
$$

No caso do modelo de Kane, duas definições diferentes dos parâmetros de Luttinger são usadas na literatura analisada ${ }^{[58,60,61\}}$. Ambas usam expressões do tipo das eqs. (2.112) a (2.114), porém uma com as constantes $L, M$ e $N$ e outra com as constantes $\bar{L}, \bar{M}$ e $\bar{N}$. No primeiro caso a definição dos parâmetros de Luttinger em termos dos elementos da matriz de momentos é a mesma do modelo de Luttinger-Kohn, nesse caso usaremos a notação já estabelecida $\gamma_{1}, \gamma_{2}$ e $\gamma_{3}$. No segundo caso a definição difere da do modelo de Luttinger-Kohn e usaremos a notação $\overline{\gamma_{1}}, \overline{\gamma_{2}}$ e $\overline{\gamma_{3}}$. Dessa forma teremos:

$$
\begin{aligned}
& \overline{\gamma_{1}}=-\frac{2 m_{0}}{\hbar^{2}} \frac{1}{3}(\bar{L}+2 \bar{M}) \\
& \overline{\gamma_{2}}=-\frac{2 m_{0}}{\hbar^{2}} \frac{1}{6}(\bar{L}-\bar{M}) \\
& \overline{\gamma_{3}}=-\frac{2 m_{0}}{\hbar^{2}} \frac{1}{6} \bar{N} .
\end{aligned}
$$

Determinando a relação entre os parâmetros de Luttinger antigos e novos, obteremos as expressōes:

$$
\begin{aligned}
\gamma_{1} & =\overline{\gamma_{1}}+\frac{1}{3} \frac{2 m_{0}}{\hbar^{2}} \frac{P^{2}}{E_{g}} \\
\gamma_{2} & =\overline{\gamma_{2}}+\frac{1}{6} \frac{2 m_{0}}{\hbar^{2}} \frac{P^{2}}{E_{g}} \\
\gamma_{3} & =\overline{\gamma_{3}}+\frac{1}{6} \frac{2 m_{0}}{\hbar^{2}} \frac{P^{2}}{E_{g}}
\end{aligned}
$$




\subsubsection{Massa efetiva em termos dos parâmetros de Kane}

O Hamiltoniano de Kane pode ser expresso por ambos os tipos de parâmetros e o mesmo se dá para as bandas de energia e suas massas efetivas. No GaAs temos 4 bandas bidegeneradas, a banda de condução $(c)$, as bandas de valência de buracos leves $(l h)$ e pesados $(h h)$ e a banda de desdobramento dos estados spin-órbita (so), cujas curvas de dispersão $E_{\nu}(\mathbf{k}), \nu=c, l h, h h, s o$, são, em geral, anisotrópicas e nãoparabólicas. Fixando uma determinada direção do vetor de onda $\mathbf{k}$, e desprezando os termos não-parabólicos em $\mathbf{k}$, massas efetivas $m_{\nu \mathbf{k}}^{*}$ podem ser definidas. Para as duas direções de alta simetria [100] e [111], há seis massas independentes, $m_{e}^{*} \equiv m_{e[100]}^{*}=m_{e[111]}^{*}, m_{l h[100]}^{*}$, $m_{l h[111]}^{*}, m_{h h[100]}^{*}, m_{h h[111]}^{*}, m_{s o}^{*} \equiv m_{s o[100]}^{*}=m_{s o[111]}^{*}$. As expressões abaixo podem ser deduzidas para essas massas, em termos dos antigos parâmetros de Luttinger, diagonalizando-se o Hamiltoniano de Kane:

$$
\begin{array}{ll}
m_{e}^{*-1} & =A+\frac{E_{g}+\frac{2}{3} \Delta}{E_{g}+\Delta} \frac{E_{p}}{E_{g}} \\
m_{h h[100]}^{*} & =\gamma_{1}-2 \gamma_{2} \\
m_{h h[111]}^{*} & =\gamma_{1}-2 \gamma_{3} \\
m_{l h[100]}^{*} & =\gamma_{1}+2 \gamma_{2} \\
m_{l h[111]}^{*}-1 & =\gamma_{1}+2 \gamma_{3} \\
m_{s o}^{*-1} & =\gamma_{1}-\frac{1}{3} \frac{\Delta}{E_{g}+\Delta} \frac{E_{p}}{E_{g}}
\end{array}
$$

As massas efetivas estão expressas em termos da massa do elétron livre $m_{0}$, o 'gap' em $\Gamma$ é definido como $E_{g}=E_{s}+\frac{\Delta}{3}$ e $E_{p}=\left(2 m_{0} / \hbar^{2}\right) P^{2}$ é um parâmetro de energia dependente do elemento $P$ da matriz de momentos. $P$ sendo real, $E_{p}>0$. As relações (2.124) a (2.129) concordam com as dadas na ref. [61]. Em relação à direção [110] temos duas novas massas independentes, $m_{l h(110]}^{*}$ e $m_{h h[110]}^{*}$, enquanto as outras, $m_{e[110]}^{*} \mathrm{e}$ $m_{s o[110]}^{*}$, têm os mesmos valores que as das direções [100] e [111]. As 
massas independentes são dadas pelas expressões:

$$
\begin{aligned}
& \left.m_{h h[110]}^{*}\right]^{-1}=\gamma_{1}-\sqrt{\gamma_{2}^{2}+3 \gamma_{3}^{2}} \\
& \left.m_{l h[110]}^{*}\right]^{-1}=\gamma_{1}+\sqrt{3 \gamma_{2}^{2}+\gamma_{3}^{2}} .
\end{aligned}
$$

Se colocarmos as massas em termos dos novos parâmetros de Luttinger, teremos:

$$
\begin{aligned}
& m_{e}^{*-1}=A+\frac{E_{g}+\frac{2}{3} \Delta}{E_{g}+\Delta} \frac{E_{p}}{E_{g}} \\
& m_{h h[100]}^{*}=\overline{\gamma_{1}}-2 \overline{\gamma_{2}} \\
& m_{h h[111]}^{* 1}=\overline{\gamma_{1}}-2 \overline{\gamma_{3}} \\
& m_{l h[100]}^{*-1}=\overline{\gamma_{1}}+2 \overline{\gamma_{2}}+\frac{2}{3} \frac{E_{p}}{E_{g}} \\
& m_{l h[111]}^{*}=\overline{\gamma_{1}}+2 \overline{\gamma_{3}}+\frac{2}{3} \frac{E_{p}}{E_{g}} \\
& m_{s o}^{*-1}=\overline{\gamma_{1}}+\frac{E_{p}}{3\left(E_{g}+\Delta\right)} .
\end{aligned}
$$

Essas equações concordam com as da ref. [58].

\subsubsection{Relações entre as massas efetivas}

Os conjuntos de equações (2.124) a (2.129) e (2.132) a (2.137) implicam em relaçōes entre as várias massas efetivas. Para as massas de buracos leves e pesados nas duas direções de alta simetria temos:

$$
m_{h h[100]}^{* 1}+m_{l h[100]}^{* 1}-m_{h h[111]}^{* 1}-m_{l h[111]}^{* 1}=0 .
$$

Pode ser mostrado que a mesma relação pode ser deduzida para quaisquer duas direções $\mathbf{k}$ e $\mathbf{k}^{\prime}$, ou seja, a soma $m_{h h \mathbf{k}}^{*}{ }^{-1}+m_{l h \mathbf{k}}^{*}{ }^{-1}$ é independente da direção $\mathbf{k}$. 
A relação (2.138) pode ser vista como um teste da adequação de um dado conjunto de massas experimentais, se o modelo de Kane for aplicável ao sistema a que eles se referem, ou como um teste da aplicabilidade do modelo de Kane, se as massas forem confiáveis. Se a relação da eq. (2.138) for violada apreciavelmente por um certo conjunto confiável de massas experimentais, o modelo de Kane não é aplicável. Aqui, apreciavelmente significa que o desvio numérico do lado esquerdo da eq. (2.138) é da ordem de 1. Para quantificar, vamos definir o número de erro de Kane como:

$$
\delta_{K} \equiv m_{h h[100]}^{*}+m_{l h[100]}^{*}-m_{h h[111]^{-1}}^{*}-m_{l h[111]}^{*}
$$

Além disso, podemos determinar também a relação:

$$
m_{h h[100]}^{* 1}+m_{l h[100]}^{*-1}-2 m_{s o}^{*-1}=\frac{2}{3} \frac{\Delta}{E_{g}+\Delta} \frac{E_{p}}{E_{g}}
$$

Como $E_{p}$ não pode ser negativo, no modelo de Kane, temos a inequação:

$$
m_{h h[100]}^{*}+m_{l h(100]}^{*}-2 m_{s o}^{*-1}>0 .
$$

Como no caso da relação (2.138), sua violação por um conjunto confiável de massas experimentais indica a não-aplicabilidade do modelo de Kane. Se $E_{p}=0$, o modelo de Kane se transforma no modelo de Luttinger-Kohn. Então uma nova equação se aplica:

$$
m_{h h[100]}^{*}+m_{l h[100]}^{*}-2 m_{s o}^{*-1}=0 .
$$

A equação (2.142) tem um significado para o modelo de LuttingerKohn semelhante ao que a equação (2.138) tem com relação ao modelo de Kane. Se a relação que ela apresenta for violada apreciavelmente por um certo conjunto confiável de massas experimentais, o modelo de Luttinger-Kohn não é aplicável. Para quantificar vamos definir o número de erro de Luttinger-Kohn como:

$$
\delta_{L K} \equiv m_{h h[100]}^{*}+m_{l h[100]}^{*}-2 m_{s o}^{*-1} \text {. }
$$




\subsubsection{Cálculo dos parâmetros de Kane}

Vamos assumir que as 6 massas das equações (2.124) a (2.129) ou (2.132) a (2.137) são conhecidas. Se $E_{g}$ e $\Delta$ estão também disponíveis, como vamos assumir aqui, então os 5 parâmetros do modelo de Kane podem ser determinados através de 5 das 6 massas das equações (2.124) a (2.129) ou (2.132) a (2.137). Vamos omitir de nosso cálculo a massa $m_{l h[100]}^{*}$, i.e., as eq. (2.136) ou (2.128). As soluções numéricas para os conjuntos restantes das eqs. (2.124) a (2.129) ou (2.132) a (2.137) são dados na tabela 2.1 para $E_{g}=1,1519 \mathrm{eV}$ e $\Delta=0,341 \mathrm{eV}$, usando três conjuntos ligeiramente diferentes de massas efetivas. Os primeiros dois conjuntos são massas experimentais extraídas de [61] (a) e de [62] (b). No terceiro conjunto (c), a massa do elétron é a mesma que nos dois conjuntos anteriores, mas as massas relativas à banda de valência são calculadas por meio do modelo de Luttinger-Kohn usando os parâmetros de Luttinger $\gamma_{1}=6,85, \gamma_{2}=2,1$ e $\gamma_{3}=2,9$ extraídos da ref. [63]. Uma análise inicial da tabela 2.1 mostra que o número de erro de Kane $\delta_{K}$ é pequeno para os três conjuntos de dados, portanto o modelo de Kane é aplicável. O número de erro de Luttinger-Kohn $\delta_{L K}$ é diferente de zero para os conjuntos (a) e (b) e praticamente zero para o conjunto (c), o que é esperado, já que este conjunto foi determinado a partir do modelo de Luttinger-Kohn. Na tabela 2.1 pode-se perceber que, apesar das massas efetivas utilizadas serem muito próximas, os parâmetros $A, E_{p}, \overline{\gamma_{1}}, \overline{\gamma_{2}}$ e $\overline{\gamma_{3}}$ têm valores substancialmente diferentes para cada conjunto diferente de massas efetivas.

Para entender a razão dessa grande diferença, vamos estudar as soluções dos conjuntos de equações lineares (2.124) a (2.129) e (2.132) a (2.137) na sua forma mais geral. Para $E_{p}$ temos:

$$
E_{p}=\frac{3}{2} \frac{E_{g}\left(E_{g}+\Delta\right)}{\Delta}\left(m_{h h[100]}^{*}{ }^{-1}+m_{l h[100]}^{*}{ }^{-1}-2 m_{s o}^{*}{ }^{-1}\right) .
$$

Os outros parâmetros são expressos em termos de $E_{p}$. Para $A$ obtemos:

$$
A=m_{e}^{*-1}-\frac{\left(E_{g}+\frac{2}{3} \Delta\right)}{E_{g}+\Delta} \frac{E_{p}}{E_{g}} .
$$


Os antigos parâmetros de Luttinger-Kohn são dados por:

$$
\begin{aligned}
\gamma_{1} & =m_{s o}^{*-1}+\frac{1}{3} \frac{\Delta}{E_{g}+\Delta} \frac{E_{p}}{E_{g}} \\
\gamma_{2} & =\frac{1}{2}\left(m_{s o}^{*-1}-m_{h h[100]}^{*}\right)+\frac{1}{6} \frac{\Delta}{E_{g}+\Delta} \frac{E_{p}}{E_{g}} \\
\gamma_{3} & =\frac{1}{2}\left(m_{s o}^{*-1}-m_{h h[111]}^{*}\right)+\frac{1}{6} \frac{\Delta}{E_{g}+\Delta} \frac{E_{p}}{E_{g}}
\end{aligned}
$$

e os novos por:

$$
\begin{aligned}
\overline{\gamma_{1}} & =m_{s o}^{*-1}-\frac{1}{3} \frac{E_{p}}{E_{g}+\Delta}, \\
\overline{\gamma_{2}} & =\frac{1}{2}\left(m_{s o}^{*-1}-m_{h h[100]}^{*}\right)-\frac{1}{6} \frac{E_{p}}{E_{g}+\Delta}, \\
\overline{\gamma_{3}} & =\frac{1}{2}\left({m_{s o}^{*}}^{-1}-m_{h h[111]}^{*}\right)-\frac{1}{6} \frac{E_{p}}{E_{g}+\Delta} .
\end{aligned}
$$

Como era de se esperar, o parâmetro de energia de Kane $E_{p}$ é proporcional ao número de erro de Luttinger-Kohn $\delta_{L K}$ e, para o modelo de Luttinger-Kohn ambos, $E_{p}$ e $\delta_{L K}$, têm valor zero. A eq. (2.144) mostra a razão pela qual apesar da pequena diferença das massas efetivas para os valores que obedecem Luttinger-Kohn (eq. (2.142)), $E_{p}$ assume valores altos. Em primeiro lugar, os valores das massas do buraco leve e do buraco 'split-off' são pequenas quando comparadas a 1, o que resulta geralmente em números de erro $\delta_{L K}$ maiores que 1 . Em segundo lugar, o fator que multiplica $\delta_{L K}$ em $E_{p}$ é grande quando comparado a $E_{g}$ se $\Delta \ll E_{g}$ (para o GaAs ele é $15 \mathrm{eV}$, ou seja aproximadamente 10 vezes $E_{g}$ ). Dessa maneira $E_{p}$, em unidades de $E_{g}$, pode ser muito grande mesmo que as diferenças entre as massas usadas e as massas que obedecem à relação de Luttinger-Kohn sejam pequenas.

Os altos valores de $E_{p}$ explicam porque o parâmetro A da eq. (2.145) e os novos parâmetros de Luttinger $\overline{\gamma_{1}}, \overline{\gamma_{2}}$ e $\overline{\gamma_{3}}$ das eqs. (2.149) a (2.151) são tão sensíveis a variações nas massas efetivas. Isto explica também a grande diferença entre os novos parâmetros de Luttinger $\overline{\gamma_{1}}, \overline{\gamma_{2}}$ e $\overline{\gamma_{3}}$ e os antigos $\gamma_{1}, \gamma_{2}$ e $\gamma_{3}$. Os últimos são relativamente estáveis com relação 


\begin{tabular}{|l|ccc|}
\hline \hline & Conjunto (a) & Conjunto (b) & Conjunto (c) \\
\hline$m_{e}^{*}$ & 0.0665 & 0.0593 & 0.0665 \\
$m_{h h[100]}^{*}$ & 0.340 & 0.377 & 0.377 \\
$m_{l h[100]}^{*}$ & 0.094 & 0.091 & 0.0904 \\
$m_{h h[111]}^{*}$ & 0.816 & 0.952 & 0.952 \\
$m_{l h[111]}^{*}$ & 0.0809 & 0.0791 & 0.079 \\
$m_{s o}^{*}$ & 0.178 & 0.172 & 0.146 \\
\hline$\delta_{K}$ & -0.0069 & -0.0511 & 0.00581 \\
$\delta_{K L}$ & 2.343 & 2.0136 & 0.0158 \\
\hline$A$ & -2.96 & 1.34 & 14.86 \\
$E_{p}$ & 29.12 & 25.15 & 0.119 \\
$\bar{\gamma}_{1}$ & 0.398 & 1.31 & 6.822 \\
$\bar{\gamma}_{2}$ & -1.271 & -0.67 & 2.085 \\
$\bar{\gamma}_{3}$ & -0.414 & 0.13 & 2.886 \\
$\gamma_{1}$ & 6.790 & 6.821 & 6.857 \\
$\gamma_{2}$ & 1.924 & 2.084 & 2.102 \\
$\gamma_{3}$ & 2.780 & 2.885 & 2.903 \\
\hline \hline
\end{tabular}

Tabela 2.1: Parâmetros do modelo de Kane calculados através das equações (2.144) a (2.151) com diferentes conjuntos de massas efetivas: conjunto (a) segundo Gershoni et al. ${ }^{[61]}$, conjunto (b) segundo Ram-Mohan et al. ${ }^{[62]}$ e conjunto (c) com a mesma massas $m_{e}^{*}$ do conjunto (a) e massas efetivas para a banda de valência calculadas dentro da aproximação de Luttinger-Kohn, usando os parâmetros de Luttinger extraídos de Harbeke et al. ${ }^{[63]}\left(\gamma_{1}=6,85, \gamma_{2}=2,1\right.$ e $\gamma_{3}=2,9$, massas em unidades de $\left.m_{0}\right)$ e os valores para o 'gap' $E_{g}=1,519 \mathrm{eV}$ e para o desdobramento da banda 'split-off' de $\Delta=0,341 \mathrm{eV}$. 
a mudanças nas massas efetivas. Seus valores no modelo de Kane são próximos aos valores no modelo de Luttinger-Kohn, pois os mesmos são calculados a partir das relações:

$$
\begin{aligned}
\gamma_{1} & =\frac{1}{2}\left(m_{l h[100]}^{*}+m_{h h[100]}^{*}\right), \\
\gamma_{2} & =\frac{1}{4}\left(m_{l h[100]}^{*}-m_{h h[100]}^{*}\right), \\
\gamma_{3} & =\frac{1}{4}\left(m_{l h[111]}^{*}-m_{h h[111]}^{*}\right),
\end{aligned}
$$

por meio das massas experimentais $m_{l h[100]}^{*}, m_{h h[100]}^{*}, m_{l h[111]}^{*}$ e $m_{h h[111]}^{*}$.

Para se deduzir as equações (2.152) a (2.154), parte-se das eqs. (2.146) a (2.148), fazendo $E_{p}=0$ e eliminando $m_{s o}^{*}$ por meio da condição de Luttinger-Kohn (2.142). Os valores obtidos dessa maneira são $\gamma_{1}=6,79, \gamma_{2}=1,92, \gamma_{3}=2,78$ para o conjuntos (a) da tabela 2.1 e $\gamma_{1}=6,82, \gamma_{2}=2,08, \gamma_{3}=2,89$ para o conjunto (b) da mesma tabela. Se as expressões (2.132) a (2.137) tivessem sido usadas nesse cálculo, fazendo-se $E_{p}=0$ e substituindo-se $m_{s o}^{*}$ por seu valor experimental, o parâmetro $\gamma_{1}$ teria o valor 5,62 para o conjunto (a) e 5,81 para o conjunto (b), uma diferença remarcável em relação aos valores anteriores. Os parâmetros $\gamma_{2}$ e $\gamma_{3}$ mantêm os mesmos valores anteriores. Essa discrepância é causada pela violação da condição de Luttinger-Kohn (2.142) pelos dois conjuntos de massas experimentais. Portanto o modelo de Luttinger-Kohn não pode ser aplicado a esses conjuntos. Caso se queira utilizar esse modelo, no entanto, deve-se utilizar apenas as bandas de buracos leves e pesados, deixando de fora a banda de buracos 'split-off'. Como neste caso não há, no Hamiltoniano, outros termos que dependem diretamente de $E_{p}$, os erros e ambigüidades decorrentes da aproximação podem ser desprezados. 


\section{Capítulo 3}

\section{Cálculo Autoconsistente da Estrutura de Bandas}

O método $\mathbf{k} \cdot \mathbf{p}$ é uma ferramenta poderosa no cálculo da estrutura de bandas de um material volumétrico ('bulk'). Sistemas baseados em poços $\delta$-doping são unidimensionais e têm uma alta densidade de portadores localizados em regiões específicas das amostras. A autoorganização desses portadores dá origem a dois novos potenciais, um decorrente da interação coulombiana de uma partícula $\left(V_{C}\right)$ e outro da interação de troca e correlação $\left(V_{X C}\right)$. Portanto usando a aproximação de um elétron escrevemos seu novo Hamiltoniano como:

$$
H=H_{0}+V_{C}(\mathbf{x})+V_{X C}(\mathbf{x})
$$

onde o potencial de uma partícula é a soma do potencial devido às impurezas $\left(V_{I}\right)$ com o potencial de uma partícula para os portadores $\left(V_{H}\right)$, também chamado de potencial de Hartree por ser escrito a partir da aproximação de Hartree, que vai ser definida adiante. Dessa maneira:

$$
V_{C}(\mathbf{x})=V_{I}(\mathbf{x})+V_{H}(\mathbf{x})
$$

Tomando os spinores na forma de Bloch, com $\mathbf{k}$ sendo um vetor da primeira zona de Brillouin, $s$ a coordenada de spin e $\nu$ o índice da 
banda, escrevemos a equação de Schrödinger como:

$$
H(\mathbf{x} s \mid \nu \mathbf{k})=\left[H_{0}+V_{C}(\mathbf{x})+V_{X C}(\mathbf{x})\right](\mathbf{x} s \mid \nu \mathbf{k})=E_{\nu}(\mathbf{k})(\mathbf{x} s \mid \nu \mathbf{k}) .
$$

A ferramenta adequada para se determinar efetivamente esses novos potenciais é o método da autoconsistência. Por ele resolve-se a equação de Schrödinger para um potencial tentativo inicial. Com as autofunções calculadas, determina-se a distribuição de cargas para o sistema e o potencial gerado por ela, através da integral dupla da equação de Poisson:

$$
\nabla^{2} V_{C}(\mathbf{x})=-\frac{4 \pi e}{\epsilon} \rho(\mathbf{x})
$$

Seguindo o procedimento de separar o potencial de uma partícula em duas partes, separamos a densidade de cargas na densidade de cargas dos portadores $(e \cdot p(\mathbf{x}))$ e das impurezas $\left(-e \cdot N_{A}(\mathbf{x})\right)$ :

$$
\rho(\mathbf{x})=e\left[p(\mathbf{x})-N_{A}(\mathbf{x})\right]
$$

Como não temos outras impurezas além dos aceitadores distribuídos em torno do plano de dopantes, a densidade total de carga no sistema representada pela soma das densidades de carga dos aceitadores e dos buracos gerados por eles (eq. (3.5)) em toda a amostra é nula. Esse argumento só é válido se tivermos uma super-rede infinita. Para o alinhamento do nível de Fermi na região do poço $\delta$-doping com o nível de Fermi no GaAs volumétrico é preciso que haja uma densidade de fundo de armadilhas para buracos. O número de buracos a ser capturado dependerá da distancia que será necessária para esse alinhamento e decairá exponencialmente com ela. Se considerarmos que o alinhamento dos níveis se dará no infinito, essa densidade torna-se nula e seu efeito na região do poço desprezível.

Com o novo potencial dá-se início a uma nova iteração. Após sucessivas iterações o potencial de entrada e o calculado através da equação de Poisson devem convergir, determinando-se assim o potencial coulombiano a que está sujeita a amostra. 
O procedimento apresentado neste capítulo vale-se do método $\mathbf{k} \cdot \mathbf{p}$ em uma formulação autoconsistente para calcular a estrutura de bandas de sistemas baseados em poços $\delta$-doping. Vamos apresentar as equações utilizadas em nosso cálculo: a forma final da equação de Schrödinger, do potencial coulombiano e do potencial de troca e correlação da maneira como foram introduzidas no programa de cálculo da estrutura de bandas.

\subsection{Célula unitária e $1^{\underline{a}}$ zona de Brillouin}

Este trabalho propõe-se a estudar super-redes de poços $\delta$-doping tipo p em GaAs crescidas na direção [001]. Assumindo-se um número par de camadas duplas Ga-As por período de super-rede e levando-se em conta a estrutura 'Zinc-Blende' do material, tem-se uma rede de Bravais tetragonal com os seguintes vetores de rede:

$$
\mathbf{A}_{1}=\frac{a}{2}\left(\mathbf{e}_{x}-\mathbf{e}_{y}\right), \quad \mathbf{A}_{2}=\frac{a}{2}\left(\mathbf{e}_{x}+\mathbf{e}_{y}\right), \quad \mathbf{A}_{3}=d \mathrm{e}_{z},
$$

onde $\mathbf{e}_{x}, \mathbf{e}_{y}, \mathbf{e}_{z}$ são os vetores unitários nas direções dos eixos do cubo, $a$ o parâmetro de rede do cristal 'zinc blende', e $d=\frac{1}{2} N a$ o parâmetro da super-rede na direção de crescimento. Será assumido que a super-rede seja infinita estendendo-se por todo o espaço em todas as direções e serão usadas condições de contorno periódicas para que se possa trabalhar sobre uma região de periodicidade finita. Os vetores que definem esta região são $G_{\|} \mathbf{A}_{1}, G_{\|} \mathbf{A}_{2}$, e $G_{z} \mathbf{A}_{3}$, onde $G_{\|}$e $G_{z}$ são inteiros. Uma região de periodicidade contém $G_{\|}^{2} G_{z}$ supercélulas e $G_{\|}^{2} G_{z} \cdot N$ células unitárias 'zinc blend', cada qual com volume igual a $\Omega_{0}=a^{3} / 4$. O volume da região de periodicidade é $G_{\|}^{2} G_{z} \cdot N \Omega_{0}=G_{\|}^{2} G_{z} \cdot d \cdot a^{2} / 2$.

Os vetores primitivos da super-rede no espaço recíproco são:

$$
\mathbf{B}_{1}=\frac{2 \pi}{a}\left(\mathbf{e}_{x}+\mathbf{e}_{y}\right), \quad \mathbf{B}_{2}=\frac{2 \pi}{a}\left(\mathbf{e}_{x}-\mathbf{e}_{y}\right), \quad \mathbf{B}_{3}=\left(\frac{4 \pi}{N a}\right) \mathbf{e}_{z} .
$$

A primeira zona de Brillouin (1 1 ZBB) correspondente é mostrada na fig. 3.1. Devido às condições periódicas de contorno, só são permitidos 


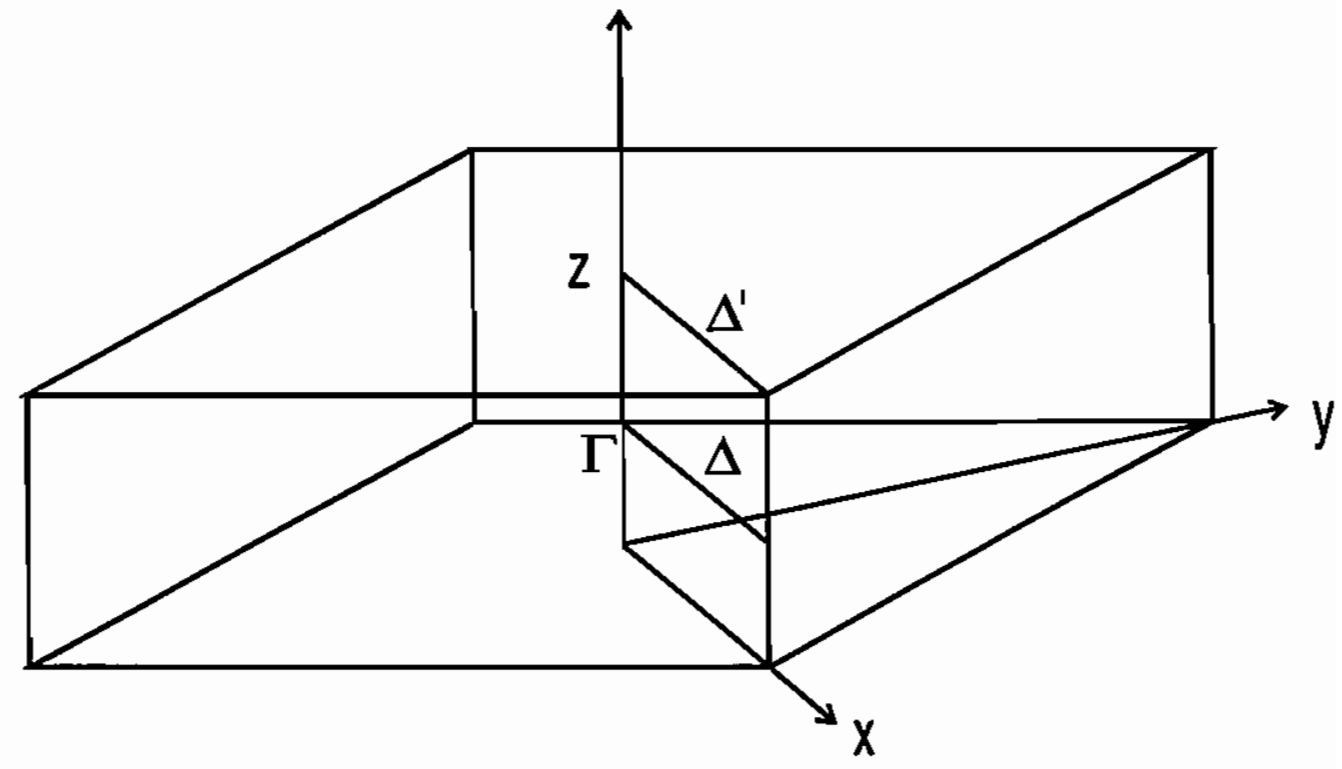

Figura 3.1: Primeira Zona de Brillouin.

valores do vetor $\mathbf{k}$ que satisfaçam a seguinte relação:

$$
\mathbf{k}=\frac{k_{1}}{G_{\|}} \mathbf{B}_{1}+\frac{k_{2}}{G_{\|}} \mathbf{B}_{2}+\frac{k_{3}}{G_{z}} \mathbf{B}_{3},
$$

onde $k_{1}, k_{2}, k_{3}$ são inteiros.

\subsection{Densidade de aceitadores, aproximação de Hartree e nível de Fermi}

Partindo do pressuposto que os átomos de Berílio em um poço $\delta$-doping se distribuem estatiscamente em torno da posição nominal do plano de dopantes, vamos assumir que o perfil de distribuição dos mesmos seja Gaussiano. Tal procedimento é sugerido por Schubert ${ }^{[1]}$ e utilizado por Koenraad et al. ${ }^{[33]}$ para através de um ajuste determinar o valor da dispersão dos dados obtidos por STM. Dessa maneira, tomando-se 
z como a direção de crescimento da amostra:

$$
N_{A}(\mathbf{x})=N_{s} \cdot \sum_{n=-\infty}^{\infty} \frac{1}{\sqrt{2 \pi} \sigma} e^{-\frac{(z-n d)^{2}}{2 \sigma^{2}}}
$$

onde $N_{s}$ é o número de aceitadores da camada e $\sigma^{2}$ a variância da distribuição Gaussiana. A raiz quadrada da variância $(\sigma)$ representa a distância média de um átomo ao centro da distribuição. A dispersão desta distribuição, dada pela largura total a meia-altura (FWHM, da sua sigla em inglês), é $\Delta z=2 \sqrt{2 \ln 2} \sigma=2.355 \sigma$. Para efeitos de comparação com perfis de distribuição retangulares, muito usados por simplificar a expressão do potencial gerado pelas impurezas, compararemos $\Delta z$ com a largura total desses perfis.

Para que possamos nos valer da autoconsistência é necessário poderse calcular a densidade de cargas de sistema $\rho(\mathbf{x})$ a partir das funções de onda dos portadores isolados. Para tanto, assume-se que a distribuição de carga dos portadores do sistema seja igual à soma das funções densidade de probabilidade dos estados ocupados. Essa é a chamada aproximação de Hartree. Em um sistema em que os portadores sejam buracos, estes estados ocupados correspondem a estados vazios de elétrons nas bandas de valência, ou seja:

$$
p(\mathbf{x})=\sum_{s} \sum_{\substack{\nu \mathbf{k} \\ \nu a z i o s}}|(\mathbf{x} s \mid \nu \mathbf{k})|^{2} .
$$

Para a determinação dos estados ocupados, é necessário se determinar o nível de Fermi $\left(E_{F}\right)$ do sistema. Estruturas baseadas em poços $\delta$-doping têm caráter eminentemente metálico, ou seja, têm bandas parcialmente ocupadas à temperatura $\mathrm{T}=0 \mathrm{~K}$. Por este motivo não podemos nos valer de pontos especiais para o cálculo do nível de Fermi ou para realizar a soma da eq. (3.10). Também não temos um sistema parabólico, caso em que poderíamos nos valer de funções analíticas para determinar $E_{F}$, como se faz para poços $\delta$-doping tipo n. A única opção que nos resta então, é determinar o nível de Fermi pela ordenação dos estados calculados através da equação de Schrödinger, e realizar a soma da eq. (3.10) em uma rede de pontos que cobre o setor irredutível da 
1ㅡㅡ Zona de Brillouin. Observando a fig. 3.1 e usando considerações de simetria podemos ver que o seu setor irredutível tem um volume $V=\frac{1}{16} V_{Z B}$, onde $V_{Z B}$ é o volume da $1^{\underline{a}}$ Zona de Brillouin. Para os cálculos executados neste trabalho foi usado um número máximo de 372 pontos na $1 \stackrel{a}{2}$ Zona de Brillouin.

Por simplicidade, foi feita a escolha de convergir o potencial à temperatura $\mathrm{T}=0 \mathrm{~K}$. Assumindo que a distribuição dos estados de buracos varie segundo a distribuição de Fermi, podemos estimar a largura de linha dos estados a diversas temperaturas. Para temperaturas da ordem de $\mathrm{T}=10 \mathrm{~K}$, essa largura é de aproximadamente $1 \mathrm{meV}$. Para uma temperatura $\mathrm{T}=60 \mathrm{~K}$, a largura é de $5 \mathrm{meV}$, e para temperaturas da ordem da temperatura ambiente $(\mathrm{T}=300 \mathrm{~K})$ corresponde a $25 \mathrm{meV}$. Cálculos anteriores da estrutura de bandas mostram que a diferença de energia entre os estados ocupados no interior do poço são maiores que $10 \mathrm{meV}$, para poços com uma densidade típica $\left(N_{A}=8 \times 10^{12} \mathrm{~cm}^{-2}\right)$. Reboredo et al. ${ }^{[35]}$ apresentam o valores de $22 \mathrm{meV}$ para a diferença entre a primeira e a segunda sub-bandas e Richards et al. ${ }^{[28]}$ uma diferença de $15 \mathrm{meV}$. Portanto, até temperaturas próximas a $100 \mathrm{~K}$, a variação da largura de linha dos estados não deve modificar consideravelmente sua ocupação.

Em conseqüência do exposto no parágrafo anterior, para temperaturas inferiores a $100 \mathrm{~K}$, pode-se calcular as ocupações dos estados, usando o valor da energia de Fermi calculado para $\mathrm{T}=0 \mathrm{~K}$, sem que essa ocupação divirja grandemente do valor real.

\subsection{Equação da massa efetiva e termo de energia cinética}

Para resolver a equação de Schrödinger (3.3), adota-se aqui a teoria da massa efetiva desenvolvida no capítulo anterior, em duas de suas formulações, as de Luttinger-Kohn ${ }^{[36]}$ na sua forma $4 \times 4$ e de Kane ${ }^{[57]}$. Escolhemos então, um conjunto ortonormalizado de funçōes de Bloch para um cristal 'Zinc-Blende' não-perturbado no ponto $\Gamma,\left(\mathbf{x} s \mid j m_{j}\right)$. 
$\mathrm{Na}$ formulação de Luttinger-Kohn teremos quatro funções degeneradas no topo da banda de valência (buracos leves e pesados), que serão indexadas como $j=\frac{3}{2}, m_{j}=\frac{3}{2}, \frac{1}{2},-\frac{3}{2},-\frac{3}{2}$. Na formulação de Kane serão oito funções, as quatro degeneradas no topo da banda de valência, com os mesmos índices, duas funções degeneradas de buracos 'split-off', também na banda de valência, indexadas como $j=\frac{1}{2}, m_{j}=\frac{1}{2},-\frac{1}{2} \mathrm{e}$ duas funções para os elétrons no fundo da banda de condução, que serão identificadas como $j=c, m_{j}=\frac{1}{2},-\frac{1}{2}$. Neste último caso foi usado o índice $c$, pois como o momento dessas partículas também é $j=\frac{1}{2}$, poderiam haver dificuldades de identificação. Sendo $\mathbf{k}$ um vetor da $1^{\mathrm{a}}$ ZB desse cristal, as funções de Luttinger-Kohn correspondentes serão:

$$
\left(\mathbf{x} s \mid j m_{j} \mathbf{k}\right)=\frac{1}{\sqrt{\Omega}} e^{i \mathbf{k} \cdot \mathbf{x}}\left(\mathbf{x} s \mid j m_{j}\right)
$$

Devido à periodicidade da super-rede somente vetores que tenham a forma $\mathbf{k}+K \mathbf{e}_{z}$ com $\mathbf{k}$ sendo um vetor da $1 \underline{a} \mathrm{ZB}$ e $K=\left(\frac{2 \pi}{d}\right) l$ vetores da super-rede recíproca ( com $l$ inteiro), são permitidos. Simplificando a notação utilizaremos $\left(\mathbf{x} s \mid j m_{j} \mathbf{k} K\right)$ no lugar de $\left(\mathbf{x} s \mid j m_{j} \mathbf{k}+K \mathbf{e}_{z}\right)$. Temos então:

$$
\left(\mathbf{x} s \mid j m_{j} \mathbf{k} K\right)=\frac{1}{\sqrt{\Omega}} e^{i\left(\mathbf{k}+K \mathbf{e}_{\mathbf{z}}\right) \cdot \mathbf{x}}\left(\mathbf{x} s \mid j m_{j}\right)
$$

Ao escrevermos as autofunções da super-rede nesta base temos:

$$
(\mathbf{x} s \mid \nu \mathbf{k})=\sum_{j m_{j} K}\left(j m_{j} \mathbf{k} K \mid \nu \mathbf{k}\right)\left(\mathbf{x} s \mid j m_{j} \mathbf{k} K\right) .
$$

Ao expandirmos a equação de Schrödinger (3.3), temos:

$\sum_{\substack{j^{\prime} m_{j}^{\prime} \\ K^{\prime}}}\left(j m_{j} \mathbf{k} K\left|H_{0}+V_{C}+V_{X C}\right| j^{\prime} m_{j}^{\prime} \mathbf{k} K^{\prime}\right)\left(j^{\prime} m_{j}^{\prime} \mathbf{k} K^{\prime} \mid \nu \mathbf{k}\right)=E_{\nu}(\mathbf{k})\left(j m_{j} \mathbf{k} K \mid \nu \mathbf{k}\right)$

Examinemos agora a matriz $\left(j m_{j} \mathbf{k} K\left|H_{0}+V_{C}+V_{X C}\right| j^{\prime} m_{j}^{\prime} \mathbf{k} K^{\prime}\right)$, do Hamiltoniano não-perturbado deste sistema. Esta matriz é diagonal com 
respeito aos vetores $K$ e $K^{\prime}$, sendo seus elementos diagonais dados pela matriz $H_{\mathbf{k} \cdot \mathbf{p}}$, seja ela a matriz de Luttinger-Kohn ou a de Kane:

$$
\left(j m_{j} \mathbf{k} K\left|H_{0}\right| j^{\prime} m_{j}^{\prime} \mathbf{k} K^{\prime}\right)=\delta_{K K^{\prime}} H_{\mathbf{k} \cdot \mathbf{p}}
$$

A matriz descrita na eq. (3.15) representa o operador energia cinética efetivo da equação da função envelope para nosso problema. Este é um operador blocodiagonal e seu tratamento não necessita de maiores cuidados. Os termos dos potenciais coulombiano e de troca e correlação serão tratados nas próximas seções.

\subsection{Potencial coulombiano}

Caso o potencial coulombiano $V_{C}(\mathbf{x})$ seja uma função suave, numa escala atômica de comprimentos, pode-se desprezar os elementos de matriz entre dois estados de Bloch $j m_{j}$ diferentes. Os elementos diagonais podem, por sua vez, ser substituídos pelos elementos de matriz entre ondas planas normalizadas:

$$
(z \mid K)=\frac{1}{\sqrt{G_{z} d}} e^{i K z}
$$

Escrevem-se então os elementos da matriz do potencial coulombiano como:

$$
\left(j m_{j} \mathbf{k} K\left|V_{C}\right| j^{\prime} m_{j}^{\prime} \mathbf{k} K^{\prime}\right)=\delta_{j j^{\prime}} \delta_{m_{j} m_{j}^{\prime}}\left(K\left|V_{C}\right| K^{\prime}\right)
$$

Vamos agora escrever a transformada de Fourier da equação de Poisson, encontrando assim a sua forma no espaço recíproco:

$$
\left(K\left|V_{C}\right| K^{\prime}\right)=\frac{4 \pi e^{2}}{\epsilon} \frac{1}{\left|K-K^{\prime}\right|^{2}}\left[\left(K\left|N_{A}\right| K^{\prime}\right)-\left(K|p| K^{\prime}\right)\right]
$$

A carga total do sistema é nula e o sistema encontra-se no equilíbrio. Para garantir a neutralidade os elementos diagonais da densidade de 
cargas total devem ser nulos, o que nos leva à relação:

$$
\left(K\left|N_{A}\right| K\right)-(K|p| K)=0 .
$$

Examinemos agora os dois termos da densidade de cargas isoladamente. A matriz $\left(K\left|N_{A}\right| K\right)$ é facilmente calculada, trata-se da transformada de Fourier da eq. (3.5):

$$
\left(K\left|N_{A}\right| K^{\prime}\right)=\left(\frac{N_{s}}{d}\right) A\left(K-K^{\prime}\right),
$$

onde:

$$
A(K)=\int_{-\infty}^{\infty} d z \cos (K z) \cdot \frac{e^{-\frac{z^{2}}{2 \sigma^{2}}}}{\sqrt{2 \pi} \sigma}
$$

O coeficiente $A(K)$, expresso em (3.21), poderia ser escrito como uma função-erro de argumentos complexos. Aqui, porém, escolhemos resolver a integral numericamente.

Para escrever a matriz $(K|p| K)$ precisamos antes de mais nada, reescrever a função probabilidade de um elétron, $|(\mathbf{x} s \mid \nu \mathbf{k})|^{2}$, na base das funções definidas em (3.13):

$$
\begin{aligned}
&|(\mathbf{x} s \mid \nu \mathbf{k})|^{2}=\sqrt{\Omega} \sum_{\substack{j^{\prime} m_{j}^{\prime} \\
k^{\prime}}} \sum_{\substack{j m_{j} \\
k}} \sum_{K^{\prime \prime} K^{\prime \prime \prime}}\left(j m_{j} \mathbf{k} K^{\prime \prime} \mid \nu \mathbf{k}\right)\left(\nu \mathbf{k} \mid j^{\prime} m_{j}^{\prime} \mathbf{k} K^{\prime \prime \prime}\right) \\
& \times\left(z \mid K^{\prime \prime}-K^{\prime \prime \prime}\right)\left(j m_{j} \mathbf{0} \mid \mathbf{x} s\right)\left(\mathbf{x} s \mid j^{\prime} m_{j}^{\prime} \mathbf{0}\right) .
\end{aligned}
$$

Se tomarmos a média dessa expressão na célula unitária do material volumétrico e somarmos sobre as diferentes coordenadas de spin, removeremos as flutuações espaciais na escala atômica de comprimentos. A média $\sum_{s} \overline{|(\mathbf{x} s \mid \nu \mathbf{k})|^{2}}$ é diagonal em $m_{j}, m_{j}^{\prime}$ e $j, j^{\prime}$, sendo os elementos diagonais dados por:

$$
\sum_{s} \overline{|(\mathbf{x} s \mid \nu \mathbf{k})|^{2}}=\sqrt{\Omega} \sum_{j m_{j}} \sum_{K^{\prime \prime} K^{\prime \prime \prime}}\left(j m_{j} \mathbf{k} K^{\prime \prime} \mid \nu \mathbf{k}\right)\left(\nu \mathbf{k} \mid j m_{j} \mathbf{k} K^{\prime \prime \prime}\right)\left(z \mid K^{\prime \prime}-K^{\prime \prime \prime}\right) .
$$


Usando a média calculada podemos exprimir a função densidade de cargas para buracos como:

$$
p(z)=\sqrt{\Omega} \sum_{\substack{\nu k \\ \text { vazios }}} \sum_{j m_{j}} \sum_{K^{\prime \prime} K^{\prime \prime \prime}}\left(j m_{j} \mathbf{k} K^{\prime \prime} \mid \nu \mathbf{k}\right)\left(\nu \mathbf{k} \mid j m_{j} \mathbf{k} K^{\prime \prime \prime}\right)\left(z \mid K^{\prime \prime}-K^{\prime \prime \prime}\right) .
$$

Portanto, na representação de ondas planas escrevemos:

$$
\left(K|p| K^{\prime}\right)=\frac{1}{\Omega} P\left(K-K^{\prime}\right)
$$

onde:

$$
P(K)=\sum_{\substack{\nu k \\ \nu \alpha x i o s}} \sum_{j m_{j}} \sum_{K^{\prime \prime}}\left(j m_{j} \mathbf{k} K^{\prime \prime} \mid \nu \mathbf{k}\right)\left(\nu \mathbf{k} \mid j m_{j} \mathbf{k} K^{\prime \prime}-K\right) .
$$

Calculadas as expressões (3.20) e (3.24) podemos, substituindo-as na eq. (3.18), escrever a expressão para o potencial coulombiano total de nosso sistema:

$$
\left(K\left|V_{C}\right| K^{\prime}\right)=\frac{4 \pi e^{2}}{\epsilon} \frac{1}{\left|K-K^{\prime}\right|^{2}}\left[\frac{N_{s}}{d} A\left(K-K^{\prime}\right)-\frac{1}{\Omega} P\left(K-K^{\prime}\right)\right] .
$$

\subsection{Potencial de troca e correlação}

O potencial de troca e correlação é fundamental no tratamento de sistemas baseados em estruturas $\delta$-doping tipo p em GaAs. Por ter portadores muito localizados, o potencial coulombiano devido à camada de dopantes de poços tipo p sofre uma blindagem efetiva muito maior que a de seus congêneres tipo $\mathrm{n}$. Por depender basicamente da quantidade de portadores presentes na amostra, a importância do termo de troca e correlação em relação ao potencial coulombiano é muito maior em poços tipo p que em poços tipo n de mesma concentração. 
Vamos deduzir uma expressão para o termo de troca e correlação de um gás de buracos inomogêneo através de dois passos distintos, baseandonos na ref. [64], que propunha a solução deste problema para o estudo Silício e Germânio volumétrico. Em primeiro lugar determinaremos uma expressão para um gás de buracos homogêneo, para em seguida, utilizando a aproximação da densidade local determinarmos a expressão do gás de buracos inomogêneo.

\section{Potencial para um gás homogêneo de buracos}

Expressões parametrizadas dentro da aproximação de densidade local são usualmente utilizadas para descrever o termo de troca e correlação em sistemas com um gás de elétrons inomogêneo em poços $\delta$-doping tipo n. A ref. [65] é uma revisão das diversas aproximações. Em poços $\delta$-doping tipo n a parametrização mais utilizada é a proposta por Hedin e Lundqvist ${ }^{[34]}$. Para um gás homogêneo de elétrons de massa efetiva $m^{*}$, com densidade $n$, num material de constante dielétrica $\epsilon$, o potencial de troca e correlação é descrito pela expressão:

$$
V_{X C}=-\frac{e^{2}}{2 \epsilon a_{B}^{*}}\left(\frac{2}{\pi \alpha r_{s}}\right)-\frac{e^{2}}{2 \epsilon a_{B}^{*}}\left(\frac{2}{\pi \alpha}\right) 0.0368 \ln \left(1+\frac{21}{r_{s}}\right) .
$$

onde $a_{B}^{*}$, representa o raio de Bohr efetivo:

$$
a_{B}^{*}=\epsilon\left(m_{0} / m^{*}\right) a_{B}
$$

$\alpha$ a constante numérica definida por:

$$
\alpha=(4 / 9 \pi)^{1 / 3}
$$

e $r_{s}$, o raio de blindagem ('screening radius'):

$$
r_{s}^{-1}=\left[(4 \pi / 3) a_{B}^{* 3} n\right]^{1 / 3}
$$

Segundo seus autores o primeiro termo da expressão (3.28) (que depende de $\left.r_{s}^{-1}\right)$, reflete a correlação de troca ou estatística, e o segundo termo (que depende de $\ln \left(1+21 / r_{s}\right)$ ), a correlação coulombiana. 
Não se pode, no entanto, usar a expressão obtida para tratar o potencial de troca e correlação para um gás de elétrons para tratar um gás de buracos. A razão é simples: esta expressão foi proposta para um sistema com um único tipo de portador. Em estruturas $\delta$-doping em $\mathrm{GaAs}$, o gás de buracos é formado por dois tipos diferentes de portadores, buracos leves e pesados.

Vamos rever a interpretação dos diferentes termos da eq. (3.28): o segundo termo, a correlação coulombiana, relaciona todos os portadores presentes no sistema. O primeiro termo, o termo de troca, porém, indica um tipo de interação que apenas portadores de mesmo tipo podem ter entre si. Isto significa que ao escrever-se a expressão do potencial de troca e correlação para um tipo particular de buracos o raio de blindagem do primeiro termo, que depende da densidade eletrônica e do raio de Bohr efetivo de um elétron $a_{B}^{*}$, deve ser substituído por um raio de blindagem que dependa da densidade parcial e do raio de Bohr daquele tipo específico de buraco enquanto no segundo termo o raio de blindagem deve ser construído a partir da densidade total de buracos e do raio de Bohr efetivo do buraco e não a partir da densidade eletrônica e do raio de Bohr eletrônico. Com isso obtemos:

$$
\begin{aligned}
& V_{X C \frac{3}{2}}=-\frac{e^{2}}{2 \epsilon a_{B \frac{3}{2}}^{*}}\left(\frac{2}{\pi \alpha r_{s \frac{3}{2}}}\right)-\frac{e^{2}}{2 \epsilon a_{B \frac{3}{2}}^{*}}\left(\frac{2}{\pi \alpha}\right) 0.0368 \ln \left(1+\frac{21}{R_{s \frac{3}{2}}}\right), \\
& V_{X C \frac{1}{2}}=-\frac{e^{2}}{2 \epsilon a_{B \frac{1}{2}}^{*}}\left(\frac{2}{\pi \alpha r_{s \frac{1}{2}}}\right)-\frac{e^{2}}{2 \epsilon a_{B \frac{1}{2}}^{*}}\left(\frac{2}{\pi \alpha}\right) 0.0368 \ln \left(1+\frac{21}{R_{s \frac{1}{2}}}\right),
\end{aligned}
$$

onde

$$
\begin{aligned}
a_{B|m|}^{*} & =\epsilon\left(\frac{m_{0}}{m_{|m|}^{*}}\right) a_{B} \\
r_{s|m|} & =\left(\frac{4 \pi}{3} a_{B|m|}^{* 3} p_{|m|}\right)^{1 / 3} \\
R_{s|m|} & =\left(\frac{4 \pi}{3} a_{B|m|}^{* 3} p\right)^{1 / 3},
\end{aligned}
$$

$\operatorname{com}|m|=\frac{1}{2}, \frac{3}{2}$. 
As densidades parciais devem portanto ser determinadas. Há duas maneiras distintas de se determiná-las: diretamente do cálculo do nível de Fermi do sistema, se na aproximação de Hartree, ao invés de somarse para cada buraco os quadrados de suas componentes indistintamente, somar-se separadamente as componentes de buracos leves $(m=1 / 2) \mathrm{e}$ pesados $(m=3 / 2)$, ou determinando uma relação matemática simples que relacione as densidades parciais de portadores com a densidade total. A segunda alternativa é possível se utilizarmos a aproximação isotrópica, na qual as densidades dos buracos são descritas em função das massas efetivas dos mesmos:

$$
p_{|m|}^{i s o}=\frac{1}{3 \pi^{2}}\left(\frac{2 m_{|m|}^{*}}{\hbar^{2}}\right)^{3 / 2} E_{F}^{3 / 2}, \quad|m|=\frac{3}{2}, \frac{1}{2} .
$$

Usando a eq. (3.37), depois de alguma manipulação podemos escrever cada densidade parcial em função das duas massa efetivas e da outra densidade parcial. Se então utilizarmos a densidade total de buracos:

$$
p^{i s o}=p_{\frac{3}{2}}^{i s o}+p_{\frac{1}{2}}^{i s o}
$$

chegaremos a uma expressão para a relação entre as densidades parciais e a densidade total:

$$
p_{|m|}^{i s o}=\frac{m_{\frac{3}{2}}^{* 3 / 2}+m_{\frac{1}{2}}^{* 3 / 2}}{m_{|m|}^{* 3 / 2}} p^{i s o}
$$

Assumindo que a proporção do modelo isotrópico se mantenha, podemos escrever que:

$$
p_{|m|}=\frac{m_{\frac{3}{2}}^{* 3 / 2}+m_{\frac{1}{2}}^{* 3 / 2}}{m_{|m|}^{* 3 / 2}} p .
$$

O raio de blindagem para a troca torna-se então:

$$
r_{s|m|}=\frac{m_{|m|}^{* 1 / 2}}{\left(m_{\frac{3}{2}}^{* 3 / 2}+m_{\frac{1}{2}}^{* 3 / 2}\right)^{1 / 3}}\left(\frac{4 \pi}{3} a_{B|m|}^{* 3} p\right)^{1 / 3} .
$$


Para justificar a utilização do modelo isotrópico, devemos utilizar um conjunto de parâmetros confiáveis ${ }^{[63]}: \gamma_{1}=6,85, \gamma_{2}=2.1$ e $\gamma_{3}=2.9$. Ao considerarmos a banda de valência, consideramos um sistema com massas anisotrópicas. A anisotropia da banda de valência $\Gamma_{8}$ se deve ao termo de deformação ('warping') do Hamiltoniano. Vamos desconsiderar este termo, i.e., utilizar $\gamma_{2}=\gamma_{3}=2.1$, e calcular as massas efetivas na direção $z$ através do Hamiltoniano de Luttinger-Kohn. Os valores das massas calculadas dessa maneira, $0.377 m_{0}$ e $0.090 m_{0}$, são próximos o suficiente dos valores experimentais isotrópicos, $(0.475 \pm 0.148) m_{0} \mathrm{e}$ $(0.0875 \pm 0.0292) m_{0}{ }^{[63]}$ para justificarem a aproximação. Essa aproximação não significa que o 'warping' seja completamente desprezado em nossos cálculos, ele continua sendo levado em conta no termo de energia cinética (3.15), onde aliás, tem uma importância muito maior. Sem 'warping' as massas efetivas de buracos pesados $\left(m_{3 / 2}^{*}\right)$ e leves $\left(m_{1 / 2}^{*}\right)$ são descritas pelas expressões:

$$
\begin{aligned}
& m_{\frac{3}{2}}^{*}=\frac{1}{\gamma_{1}-2 \gamma_{2}} m_{0}, \\
& m_{\frac{1}{2}}^{*}=\frac{1}{2 \gamma_{2}+\gamma_{1}} m_{0} .
\end{aligned}
$$

Todo o tratamento teórico que faremos a seguir utilizará a formulação de Luttinger-Kohn do nosso problema, pois não há em nossos sistemas estados de elétrons ou buracos 'split-off' ocupados. Por conseqüência, não há portadores elétrons ou buracos 'split-off' e não pode haver troca ou correlação entre eles, nem correlação entre esses estados e os outros presentes no sistema. Seguindo o espírito de que não há elétrons ou buracos 'split-off' ocupados, assumiremos para a formulação de Kane, que os elementos da matriz de troca e correlação que relacionam os estados de buracos com estados de elétrons ou buracos 'split-off' são nulos. 


\section{Forma matricial do operador na base de Luttinger- Kohn}

Para escrever a matriz do potencial de troca e correlação precisamos primeiramente determinar a base de funçōes em que ele é diagonal. $\mathrm{O}$ intuito disto é podermos assumir que os dois gases de buracos não interagem entre si de maneira direta, mas apenas pela distribuição total de cargas. Dessa maneira, supondo-se que este potencial seja suave, os termos não-diagonais são desprezados e os termos diagonais são completamente determinados pelas expressões derivadas do potencial de Hedin-Lundqvist para um gás de elétrons (3.32) e (3.33). Escrevendo a matriz do potencial nesta base temos:

$$
\left(e_{\mu} \mathbf{k}\left|V_{X C}\right| e_{\mu^{\prime}} \mathbf{k}\right)=\left(\begin{array}{cccc}
V_{X C \frac{3}{2}} & 0 & 0 & 0 \\
0 & V_{X C \frac{1}{2}} & 0 & 0 \\
0 & 0 & V_{X C \frac{1}{2}} & 0 \\
0 & 0 & 0 & V_{X C \frac{3}{2}}
\end{array}\right)
$$

Neste ponto pode-se simplificar a expressão deste potencial assumindo que a base $\left.\mid e_{\mu} \mathbf{k}\right)$ seja a base das funções de Luttinger-Kohn $\left.\mid j m_{j} \mathbf{k}\right)$. Assim a matriz de troca e correlação deve ser somada à equação da massa efetiva na forma em que está, sem nenhuma outra transformação. Isto equivale a dizer que o potencial de troca e correlação atua sobre um sistema em que a interação spin-órbita não interfere com a distribuição espacial de suas cargas. Essa é uma simplificação imediata. Por outro lado, pode-se assumir a base em que a matriz é diagonal como ainda não determinada e determinar a matriz unitária que transforma essa base na base das funções de Luttinger-Kohn. Depois de determinada essa matriz de transformação basta aplicá-la à matriz da eq. (3.44), determinando dessa forma a matriz do potencial na base de LuttingerKohn.

Lembrando que fizemos a aproximação de comportamento isotrópico dos buracos para determinar a expressão do potencial de troca e correlação para cada portador, devemos, para ser consistentes, utilizar a matriz de Luttinger-Kohn com os parâmetros $\gamma_{2}=\gamma_{3}$. A transformação 
que diagonaliza essa matriz é:

$$
S_{m_{\frac{3}{2}} \mu}(\mathbf{k})=\left(\begin{array}{cccc}
-e^{-i \frac{3}{2} \phi} \cos \theta & -\frac{i \sqrt{3}}{2} e^{-i \frac{3}{2} \phi} \sin \theta & 0 & \frac{i}{2} e^{-i \frac{3}{2} \phi} \sin \theta \\
\frac{i \sqrt{3}}{2} e^{-\frac{i}{2} \phi} \sin \theta & e^{-\frac{i}{2} \phi} \cos \theta & -\frac{i}{2} e^{-\frac{i}{2} \phi} \sin \theta & 0 \\
0 & -\frac{i}{2} e^{\frac{i}{2} \phi} \sin \theta & e^{\frac{i}{2} \phi} \cos \theta & -\frac{i \sqrt{3}}{2} e^{\frac{i}{2} \phi} \sin \theta \\
-\frac{i}{2} e^{i \frac{3}{2} \phi} \sin \theta & 0 & -\frac{i \sqrt{3}}{2} e^{i \frac{3}{2} \phi} \sin \theta & e^{i \frac{3}{2} \phi} \cos \theta
\end{array}\right)
$$

que após aplicada sobre a matriz (3.44) nos leva a:

$$
\left(m \mathbf{k}\left|V_{X C}\right| m^{\prime} \mathbf{k}\right)=\left(\begin{array}{cccc}
Q_{X C} & S_{X C} & R_{X C} & 0 \\
S_{X C}^{*} & T_{X C} & 0 & R_{X C} \\
R_{X C}^{*} & 0 & T_{X C} & -S_{X C} \\
0 & R_{X C}^{*} & -S_{X C}^{*} & Q_{X C}
\end{array}\right),
$$

com

$$
\begin{array}{r}
Q_{X C}=\frac{1}{4}\left[V_{X C \frac{3}{2}}+3 V_{X C \frac{1}{2}}\right]\left(\frac{k_{x}^{2}+k_{y}^{2}}{k^{2}}\right)+V_{X C \frac{3}{2}}\left(\frac{k_{z}^{2}}{k^{2}}\right), \\
T_{X C}=\frac{1}{4}\left[3 V_{X C \frac{3}{2}}+V_{X C \frac{1}{2}}\right]\left(\frac{k_{x}^{2}+k_{y}^{2}}{k^{2}}\right)+V_{X C \frac{1}{2}}\left(\frac{k_{z}^{2}}{k^{2}}\right), \\
R_{X C}=-\frac{\sqrt{3}}{4}\left[V_{X C \frac{3}{2}}-V_{X C \frac{1}{2}}\right]\left(\frac{k_{x}-i k_{y}}{k}\right)^{2}, \\
S_{X C}=i \frac{\sqrt{3}}{2}\left[V_{X C \frac{3}{2}}-V_{X C \frac{1}{2}}\right]\left(\frac{\left(k_{x}-i k_{y}\right) k_{z}}{k^{2}}\right) .
\end{array}
$$

Se os potenciais de troca e correlação de buracos leves e pesados fossem idênticos, então, a matriz (3.46) seria diagonal com os elementos diagonais assumindo o valor comum de $V_{X C(1 / 2)}$ e $V_{X C(3 / 2)}$. Na realidade esses valores são distintos e bastante diferentes entre si, devido principalmente, às diferentes densidades dos gases de buracos leves e pesados. Num potencial que depende tão fortemente da distribuição dos portadores como o potencial dos sistemas aqui estudados, não há justificativa para trocar a matriz (3.46) por uma composta apenas de seus valores diagonais, a não ser que se estude somente a dispersão na direção perpendicular à direção de crescimento, onde os termos nãodiagonais desse Hamiltoniano desaparecem. 


\section{Operador de troca e correlação para um gás ino- mogêneo de buracos}

A matriz do potencial de troca e correlação obtida (3.46) se aplica a um gás de buracos da banda de valência $\Gamma_{8}$ espacialmente homogêneo. Como é usual, as inomogeneidades serão levadas em conta através da aproximação de densidade local, ou seja, usando-se as expressões (3.32) e (3.33) para gases inomogêneos também. Devido à dependência em $z$ do potencial externo a matriz do potencial de troca e correlação (3.46) também se torna dependente em $z$. Sua representação na base $\left.\mid j m_{j} \mathbf{k} K\right)$ é não-diagonal em tratando-se de $K$. Isto significa que $\left(m \mathbf{k}\left|V_{X C}\right| m^{\prime} \mathbf{k}\right)$ deve ser substituído por $\left(m \mathbf{k} K\left|V_{X C}\right| m^{\prime} \mathbf{k} K^{\prime}\right)$, assim como $Q_{X C}, T_{X C}, R_{X C}$ e $S_{X C}$ respectivamente por $\left(K\left|Q_{X C}\right| K^{\prime}\right)$, $\left(K\left|T_{X C}\right| K^{\prime}\right),\left(K\left|R_{X C}\right| K^{\prime}\right)$ e $\left(K\left|S_{X C}\right| K^{\prime}\right)$. Os últimos devem ser calculados numericamente das expressões $Q_{X C}(z), T_{X C}(z), R_{X C}(z)$ e $S_{X C}(z)$. Para se chegar a estas expressões devemos substituir $V_{X C(1 / 2)}$ e $V_{X C(3 / 2)}$ por $V_{X C(1 / 2)}(z)$ e $V_{X C(3 / 2)}(z)$ nas expressões (3.47), (3.48), (3.49) e (3.50). $V_{X C(1 / 2)}(z)$ e $V_{X C(3 / 2)}(z)$ são obtidas através da distribuição de cargas total obtida em (3.24) e das relaçōes entre as densidades relativas e a densidade total de (3.37) e (3.38). Desta maneira obtemos a matriz:

$$
\left(\begin{array}{cccc}
\left(K\left|Q_{X C}\right| K^{\prime}\right) & \left(K\left|S_{X C}\right| K^{\prime}\right) & \left(K\left|R_{X C}\right| K^{\prime}\right) & 0 \\
\left(K\left|S_{X C}^{*}\right| K^{\prime}\right) & \left(K\left|T_{X C}\right| K^{\prime}\right) & 0 & \left(K\left|R_{X C}\right| K^{\prime}\right) \\
\left(K\left|R_{X C}\right| K^{\prime}\right)^{*} & 0 & \left(K\left|T_{X C}\right| K^{\prime}\right) & -\left(K\left|S_{X C}\right| K^{\prime}\right) \\
0 & \left(K\left|R_{X C}\right| K^{\prime}\right)^{*} & -\left(K\left|S_{X C}\right| K\right)^{*} & \left(K\left|Q_{X C}\right| K\right)
\end{array}\right)
$$

onde:

$$
\begin{aligned}
& \left(K\left|Q_{X C}\right| K^{\prime}\right)=\frac{1}{4}\left[\left(K\left|V_{X C \frac{3}{2}}\right| K^{\prime}\right)+3\left(K\left|V_{X C \frac{1}{2}}\right| K^{\prime}\right)\right]\left(\frac{k_{x}^{2}+k_{y}^{2}}{k^{2}}\right)+\left(K\left|V_{X C \frac{3}{2}}\right| K^{\prime}\right)\left(\frac{k_{z}^{2}}{k^{2}}\right), \\
& \left(K\left|T_{X C}\right| K^{\prime}\right)=\frac{1}{4}\left[3\left(K\left|V_{X C \frac{3}{2}}\right| K^{\prime}\right)+\left(K\left|V_{X C \frac{1}{2}}\right| K^{\prime}\right)\right]\left(\frac{k_{x}^{2}+k_{y}^{2}}{k^{2}}\right)+\left(K\left|V_{X C \frac{1}{2}}\right| K^{\prime}\right)\left(\frac{k_{z}^{2}}{k^{2}}\right),
\end{aligned}
$$




$$
\begin{aligned}
& \left(K\left|R_{X C}\right| K^{\prime}\right)=-\frac{\sqrt{3}}{4}\left[\left(K\left|V_{X C \frac{3}{2}}\right| K^{\prime}\right)-\left(K\left|V_{X C \frac{1}{2}}\right| K^{\prime}\right)\right]\left(\frac{k_{x}-i k_{y}}{k}\right)^{2}, \\
& \left(K\left|S_{X C}\right| K^{\prime}\right)=i \frac{\sqrt{3}}{2}\left[\left(K\left|V_{X C \frac{8}{2}}\right| K^{\prime}\right)-\left(K\left|V_{X C \frac{1}{2}}\right| K^{\prime}\right)\right]\left(\frac{\left(k_{x}-i k_{y}\right) k_{z}}{k^{2}}\right) .
\end{aligned}
$$

\subsection{Estruturas das matrizes $H_{0}, V_{C}$ e $V_{X C}$}

Com a determinação da matriz que descreve o potencial de troca e correlação (3.51) do gás de buracos inomogêneo, a equação da massa efetiva (3.3) fica completamente determinada na representação de ondas planas. As matrizes dos três operadores distintos dessa equação, ou seja, $H_{0}, V_{C}$ e $V_{X C}$ têm estruturas diferentes. A matriz do operador energia cinética $H_{0}$ é não-diagonal com respeito ao momento angular $j$ e à sua projeção na direção $z m_{j}$, mas diagonal quando se trata dos vetores de onda $K$ da super-rede. A matriz do potencial coulombiano $V_{C}$ é seu oposto, é diagonal com respeito ao momento angular $j$ e à sua projeção $m_{j}$, e não-diagonal quanto aos vetores $K$ da super-rede. A matriz do potencial de troca e correlação é não-diagonal em relação a essas três grandezas. Os diagramas das figs. (3.2), (3.3) e (3.4) apresentam de forma esquemática estas estruturas. 


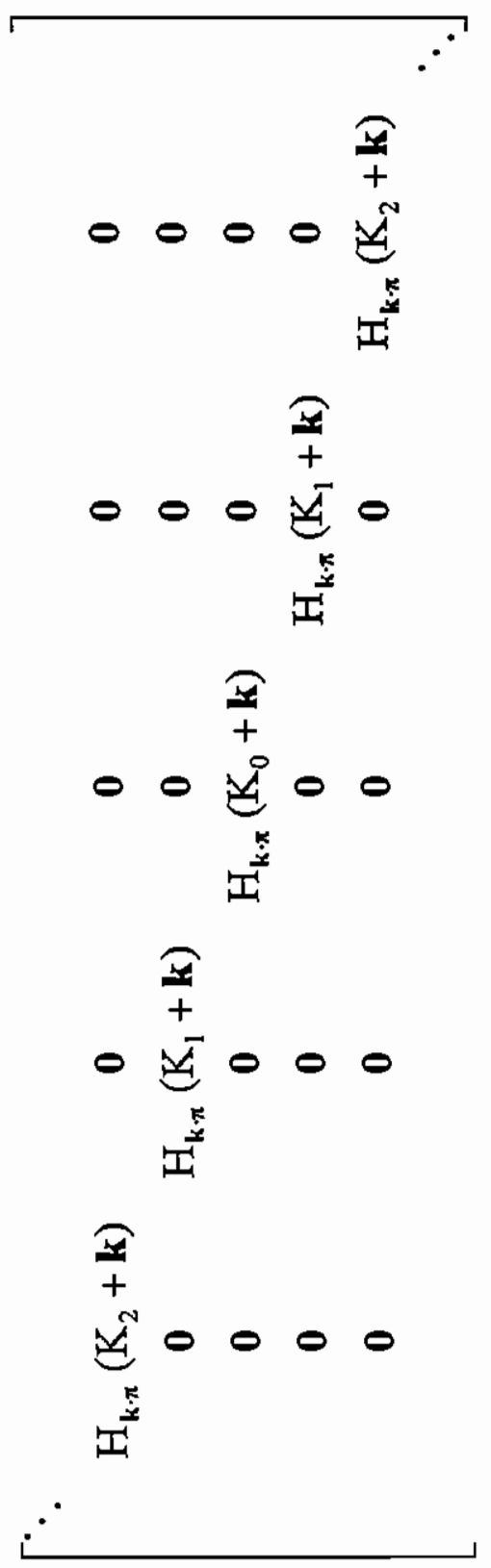

Figura 3.2: Estrutura da matriz $H_{\mathrm{k} \cdot \mathrm{p}}$. 
3. Cálculo Autoconsistente da Estrutura de Bandas

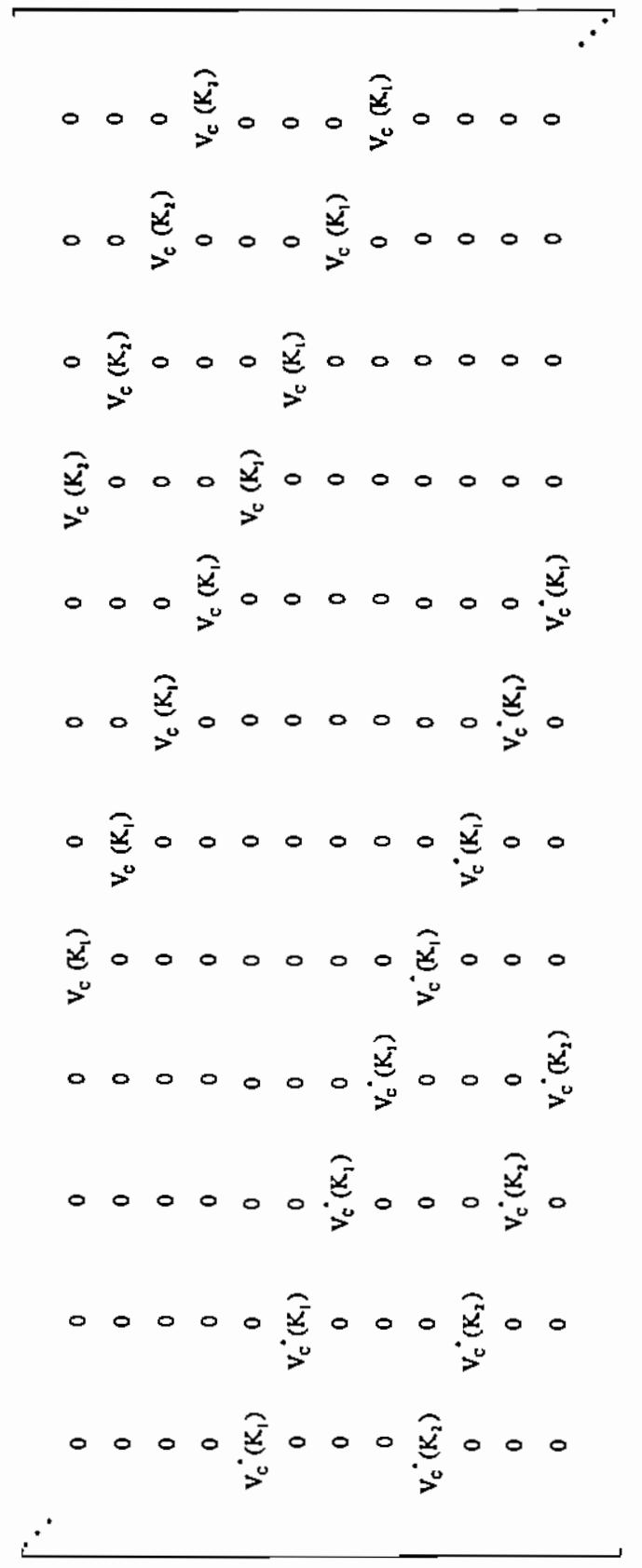

Figura 3.3: Estrutura da matriz $V_{C}$. 


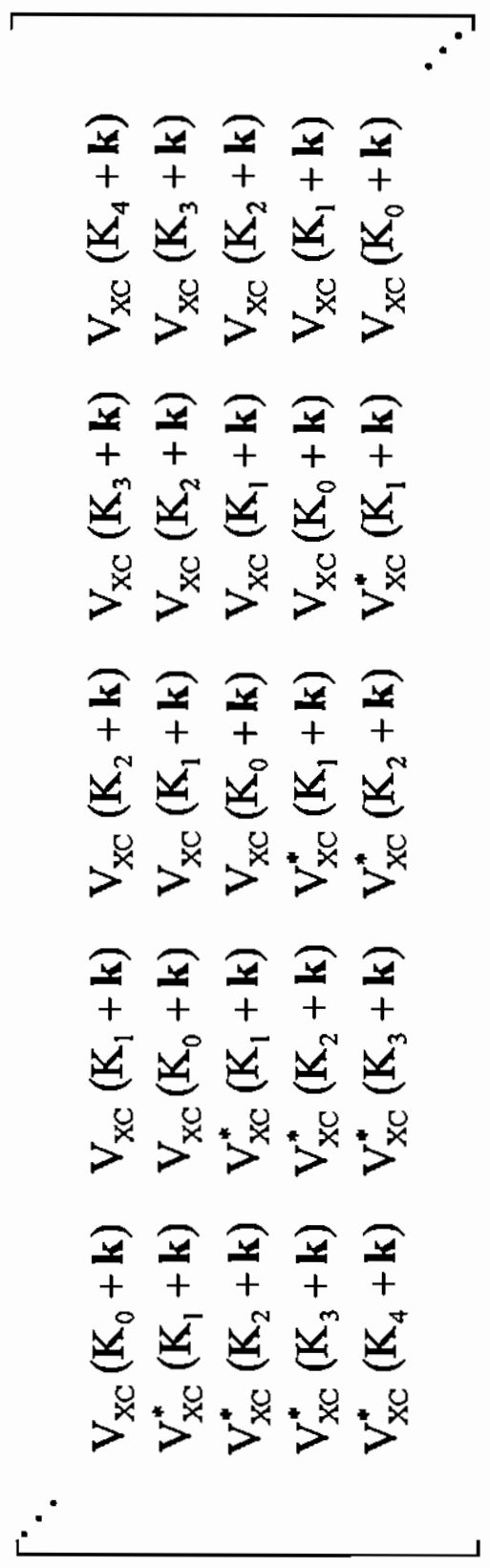

Figura 3.4: Estrutura da matriz $V_{X C}$. 


\section{Capítulo 4}

\section{Cálculo de Espectros de Luminescência}

Para testar modelos em física é necessário fazer experiências baseadas nas conclusões obtidas através dos mesmos. Nosso método para o cálculo da estrutura de bandas nos apresenta as energias das bandas, seus estados eletrônicos, a distribuição das cargas neste sistema, o potencial em que o sistema se encontra, mas não apresenta por si só uma grandeza mensurável diretamente para testá-lo. As propriedades ópticas de um material são amplamente utilizadas para a caracterização de materiais. Dentre essas propriedades, o espectro da luminescência, causada pela recombinação radiativa espontânea de portadores numa amostra, pela característica intrínseca de fornecer diferenças de energia entre os estados recombinantes, sem estados intermediários, é uma escolha natural para esse tipo de teste.

Neste capítulo apresentaremos a base teórica de um modelo que, utilizando o método apresentado nos capítulos anteriores, permite a obtenção de um espectro de luminescência para os sistemas $\delta$-doping aqui estudados. Pela necessidade de obtermos simultaneamente os estados de elétrons e de buracos, faremos uso do Hamiltoniano $8 \times 8$ de Kane para o cálculo da estrutura de bandas. Como já fizemos anteriormente, trataremos de super-redes de poços $\delta$-doping e para estudar sistemas 
de poços isolados usaremos a extrapolação para períodos de super-rede largos, caso em que dois poços vizinhos praticamente não interagem entre si.

\subsection{Uma expressão geral para a intensi- dade de luminescência}

O objeto de nossos cálculos é a emissão de luz por luminescência de uma região $\delta$-doping tipo p ou tipo n de uma amostra de GaAs que de outra maneira seria homogênea. A emissão é causada pela recombinação radiativa espontânea de portadores minoritários fora do equilíbrio por excitação óptica com portadores majoritários no equilíbrio. Os portadores minoritários encontram-se livres na amostra enquanto que os majoritários estão confinados no poço $\delta$-doping. A intensidade da luminescência destas estruturas pode ser calculada através de uma fórmula geral:

$$
I(\omega)=\frac{2 \hbar \omega^{3}}{c} \frac{e^{2}}{m_{0} c^{2}} \sum_{i f} f_{i f} N_{i}\left(1-N_{f}\right) \delta\left(E_{i}-E_{f}-\hbar \omega\right) .
$$

Onde:

$$
f_{i f}=\frac{2\left|\left(i\left|p_{x}\right| f\right)\right|^{2}}{m_{0}\left(E_{i}-E_{f}\right)}
$$

é a força de oscilador da transição de dipolo óptica entre o estado inicial $\mid i)$ e o estado final $\mid f$ ) sob luz polarizada na direção $x, E_{i}$ e $E_{f}$, os níveis de energia para os estados inicial e final e $N_{i}$ e $N_{f}$, as probabilidades de ocupação desses estados. Os estados inicial e final devem ser entendidos como os auto-estados de energia do poço $\delta$-doping. 
Essa expressão pode ainda ser melhorada se levarmos em conta o alargamento da transição devido ao tempo de recombinação médio. Substituindo a função $\delta$ da expressão (4.1) por uma Lorentziana, temos:

$$
I(\omega)=\frac{2 \hbar \omega^{3}}{c} \frac{e^{2}}{m_{0} c^{2}} \sum_{i f} f_{i f} N_{i}\left(1-N_{f}\right) \frac{1}{\pi} \frac{\gamma_{i f}}{\left(E_{i}-E_{f}-\hbar \omega\right)^{2}+\gamma_{i f}^{2}}
$$

Nesta expressão, $\gamma_{i f}$ representa o alargamento da transição devido ao tempo de vida finito.

\subsection{Estados iniciais e finais}

Para que se possa quantificar a expressão (4.3) é necessário conhecer os estados inicial e final. Para tanto utilizaremos os estados já calculados, com a teoria da massa efetiva e o método $\mathbf{k} \cdot \mathbf{p}$ na formulação $8 \times 8$ de Kane apresentados nos capítulos anteriores.

Os estados iniciais são os estados eletrônicos da banda $\Gamma_{6}$ que são caracterizados pelos números de suas minibandas eletrônicas $n_{e}=1,2, \ldots$. Os estados finais são os estados de buracos pesados (hh) e leves (lh) da banda $\Gamma_{8}$, caracterizados, respectivamente, pelo números de suas minibandas de buracos pesados $n_{h h}=1,2, \ldots$ e leves $n_{l h}=1,2, \ldots$. Os estados da banda de valência $\Gamma_{7}$ ('split-off') não são estados finais permitidos, pois encontram-se sempre ocupados. No entanto como o potencial do poço $\delta$-doping acopla esses estados com os da banda $\Gamma_{8}$, os quais são estados finais permitidos para transições radiativas, os estados da banda $\Gamma_{7}$ atuam, ainda que indiretamente, no espectro de luminescência. Como transições ópticas para estados da banda $\Gamma_{7}$ não são permitidos, podemos nos restringir aos elementos da matriz de transição $\Gamma_{6}-\Gamma_{8}$.

Os estados de uma super-rede de poços $\delta$-doping são bidegenerados por causa das combinações das simetrias de reversão temporal e inversão espacial. Vamos levar isto em consideração e introduzir números 
quânticos adicionais de spin, $\sigma_{e}$ para elétrons e $\sigma_{h h}$ e $\sigma_{l h}$ para buracos. Estes só podem assumir os valores $\uparrow$ e $\downarrow$. Dessa maneira os estados iniciais $\left.\mid \mu_{i} \mathbf{k}_{i}\right)$ dos processos de recombinação radiativa tornamse $\left.\mid n_{e} \uparrow \mathbf{k}_{e}\right)$ e $\left.\mid n_{e} \downarrow \mathbf{k}_{e}\right)$ e os estados finais $\left.\mid \mu_{f} \mathbf{k}_{f}\right)$, tornam-se por sua vez, $\left.\left.\left.\mid n_{h h} \uparrow \mathbf{k}_{h}\right), \mid n_{h h} \downarrow \mathbf{k}_{h}\right), \mid n_{l h} \uparrow \mathbf{k}_{h}\right)$ e $\left.\mid n_{l h} \downarrow \mathbf{k}_{h}\right)$. As energias de transição correspondentes $E_{n_{e}}\left(\mathbf{k}_{e}\right)-E_{n_{q h}}\left(\mathbf{k}_{h}\right)$ são independentes dos números quânticos de spin $\sigma_{e}$ e $\sigma_{q h}(q=l, h)$. Nessa notação a eq. (4.1) pode ser reescrita como:

$$
\begin{array}{r}
I(\omega)=\frac{2 \hbar \omega^{3}}{c} \frac{e^{2}}{m_{0} c^{2}} \sum_{\mathbf{k}_{e} \mathbf{k}_{h}} \sum_{n_{e}} \sum_{n_{q h}} f_{n_{e} n_{q h}}\left(\mathbf{k}_{e}, \mathbf{k}_{h}\right) N_{n_{e} \mathbf{k}_{e}}\left[1-N_{n_{q h} \mathbf{k}_{h}}\right] \times \\
\frac{\gamma_{n_{e} \mathbf{k}_{e} n_{q h} \mathbf{k}_{h}}}{\pi\left[E_{n_{e}}\left(\mathbf{k}_{e}\right)-E_{n_{q h}}\left(\mathbf{k}_{h}\right)-\hbar \omega\right]^{2}+\gamma_{n_{e} \mathbf{k}_{e} n_{q h} \mathbf{k}_{h}}^{2}} .
\end{array}
$$

O termo $f_{n_{e} n_{q h}}\left(\mathbf{k}_{e}, \mathbf{k}_{h}\right)$ indica a força de oscilador total para uma transição de um elétron para um buraco leve ou pesado.

\subsection{Força de oscilador}

Nesta seção vamos determinar a expressão que descreve $f_{n_{e} n_{q h}}\left(\mathbf{k}_{e}, \mathbf{k}_{h}\right)$, a força de oscilador total para uma transição de um elétron para um buraco leve ou pesado. Explicitando na equação (4.2) os estados inicial e final, obtemos:

$$
f_{n_{e} n_{q h}}\left(\mathbf{k}_{e}, \mathbf{k}_{h}\right)=\frac{2}{m_{0}} \sum_{\sigma_{e} \sigma_{q h}} \frac{\left|\left(n_{e} \sigma_{e} \mathbf{k}_{e}\left|p_{x}\right| n_{q h} \sigma_{q h} \mathbf{k}_{h}\right)\right|^{2}}{E_{n_{e}}\left(\mathbf{k}_{e}\right)-E_{n_{q h}}\left(\mathbf{k}_{h}\right)}
$$

Usando as funções de Luttinger-Kohn para os auto-estados da superrede $\left(s \mathbf{x} \mid j m_{j} \mathbf{k} K\right)$, pode-se escrever os elementos da matriz momento $\left(n_{e} \sigma_{e} \mathbf{k}_{e}\left|p_{x}\right| n_{q h} \sigma_{q h} \mathbf{k}_{h}\right)$ como: 


$$
\begin{aligned}
& \left(n_{e} \sigma_{e} \mathbf{k}_{e}\left|p_{x}\right| n_{q h} \sigma_{q h} \mathbf{k}_{h}\right)= \\
& \quad G^{3} \sum_{K} \sum_{j m_{j}} \sum_{j^{\prime} m_{j}^{\prime}}\left(n_{e} \sigma_{e} \mathbf{k}_{e} \mid j m_{j} K\right)\left(j m_{j}\left|p_{x}\right| j^{\prime} m_{j}^{\prime}\right)\left(j^{\prime} m_{j}^{\prime} K \mid n_{q h} \sigma_{q h} \mathbf{k}_{h}\right),
\end{aligned}
$$

onde $G^{3}=G_{z} G_{\| !}^{2}$

Ao se avaliar a expressão (4.6) deve-se levar em conta os fatos de que, no caso do GaAs, a banda de condução é apenas fracamente ligada à banda de valência e que as bandas de buracos leves e pesados só interagem fracamente com a banda de buracos 'split-off'. Aproximando, temos, portanto:

$$
\begin{gathered}
\left(\frac{3}{2} m_{\frac{3}{2}} K \mid n_{e} \sigma_{e} \mathbf{k}\right)=\left(\frac{1}{2} m_{\frac{1}{2}} K \mid n_{e} \sigma_{e} \mathbf{k}\right)=0 \\
\left(c m_{c} K \mid n_{q h} \sigma_{q h} \mathbf{k}\right)=\left(\frac{1}{2} m_{\frac{1}{2}} K \mid n_{q h} \sigma_{q h} \mathbf{k}\right)=0, \quad q=l, h .
\end{gathered}
$$

Os únicos elementos da matriz de momentos não-nulos são os que obedecem à forma $\left(c m_{c}\left|p_{x}\right| \frac{3}{2} m_{\frac{3}{2}}\right)$, onde $c$ define os estados eletrônicos da banda de condução. Esses elementos podem ser expressos em termos do elemento $\mathrm{P}$ da matriz de Kane, da seguinte maneira:

$$
\begin{gathered}
\left(c \frac{1}{2}\left|p_{x}\right| \frac{3}{2} \frac{3}{2}\right)=\frac{1}{\sqrt{2}} P, \quad\left(c \frac{\overline{1}}{2}\left|p_{x}\right| \frac{3}{2} \frac{\overline{1}}{2}\right)=\frac{i}{\sqrt{2}} P, \\
\left(c \frac{1}{2}\left|p_{x}\right| \frac{3}{2} \frac{\overline{1}}{2}\right)=\frac{1}{\sqrt{6}} P, \quad\left(c \frac{\overline{1}}{2}\left|p_{x}\right| \frac{3}{2} \frac{1}{2}\right)=\frac{i}{\sqrt{6}} P, \\
\left(c \frac{1}{2}\left|p_{x}\right| \frac{3}{2} \frac{1}{2}\right)=\left(c \frac{\overline{1}}{2}\left|p_{x}\right| \frac{3}{2} \frac{\overline{1}}{2}\right)=0 \\
\left(c \frac{1}{2}\left|p_{x}\right| \frac{3}{2} \frac{\overline{3}}{2}\right)=\left(c \frac{\overline{1}}{2}\left|p_{x}\right| \frac{3}{2} \frac{3}{2}\right)=0 .
\end{gathered}
$$


Porém, como nem todos os elementos são independentes, temos que:

$$
\begin{aligned}
& \left|\left(n_{e} \uparrow \mathbf{k}_{e}\left|p_{x}\right| n_{q h} \uparrow \mathbf{k}_{h}\right)\right|^{2}=\left|\left(n_{e} \downarrow \mathbf{k}_{e}\left|p_{x}\right| n_{q h} \downarrow \mathbf{k}_{h}\right)\right|^{2}, \quad q=l, h \\
& \left|\left(n_{e} \downarrow \mathbf{k}_{e}\left|p_{x}\right| n_{q h} \uparrow \mathbf{k}_{h}\right)\right|^{2}=\left|\left(n_{e} \uparrow \mathbf{k}_{e}\left|p_{x}\right| n_{q h} \downarrow \mathbf{k}_{h}\right)\right|^{2}, \quad q=l, h .
\end{aligned}
$$

Se tomarmos agora só os elementos independentes, teremos:

$$
\begin{aligned}
& \left|\left(n_{e} \uparrow \mathbf{k}_{e}\left|p_{x}\right| n_{q h} \uparrow \mathbf{k}_{h}\right)\right|^{2}= \\
& G^{3} \frac{|P|^{2}}{2}\left|\sum_{K}\left(n_{e} \uparrow \mathbf{k}_{e} \mid c \frac{1}{2} K\right)\left[\left(\frac{3}{2} \frac{3}{2} K \mid n_{q h} \uparrow \mathbf{k}_{h}\right)+\frac{1}{\sqrt{3}}\left(\frac{3}{2} \frac{\overline{1}}{2} K \mid n_{q h} \uparrow \mathbf{k}_{h}\right)\right]\right|^{2} \\
& \left|\left(n_{e} \downarrow \mathbf{k}_{e}\left|p_{x}\right| n_{q h} \uparrow \mathbf{k}_{h}\right)\right|^{2}= \\
& G^{3} \frac{|P|^{2}}{2}\left|\sum_{K}\left(n_{e} \downarrow \mathbf{k}_{e} \mid c \frac{\overline{1}}{2} K\right)\left[\left(\frac{3}{2} \frac{\overline{3}}{2} K \mid n_{q h} \uparrow \mathbf{k}_{h}\right)+\frac{1}{\sqrt{3}}\left(\frac{3}{2} \frac{1}{2} K \mid n_{q h} \uparrow \mathbf{k}_{h}\right)\right]\right|^{2}
\end{aligned}
$$

Ao usar esses resultados podemos expressar a força de oscilador $f_{n_{e} n_{q h}}\left(\mathbf{k}_{e}, \mathbf{k}_{h}\right)$ de uma transição elétron-buraco como:

$$
\begin{aligned}
& f_{n_{e} n_{q h}}\left(\mathbf{k}_{e}, \mathbf{k}_{h}\right)= \\
& \frac{4}{m_{0}\left[E_{n_{e}}\left(\mathbf{k}_{e}\right)-E_{n_{q h}}\left(\mathbf{k}_{h}\right)\right]}\left[\left|\left(n_{e} \uparrow \mathbf{k}_{e}\left|p_{x}\right| n_{q h} \uparrow \mathbf{k}_{h}\right)\right|^{2}+\left|\left(n_{e} \downarrow \mathbf{k}_{e}\left|p_{x}\right| n_{q h} \uparrow \mathbf{k}_{h}\right)\right|^{2}\right]
\end{aligned}
$$


Reescrevendo a eq.( 4.17) podemos evidenciar o fato que as transições levadas em conta aqui são diretas:

$$
\begin{aligned}
& f_{n_{e} n_{g h}}\left(\mathbf{k}_{e}, \mathbf{k}_{h}\right)= \\
& \frac{4}{m_{0}\left[E_{n_{e}}\left(\mathbf{k}_{e}\right)-E_{n_{q h}}\left(\mathbf{k}_{h}\right)\right]}\left[\left|\left(n_{e} \uparrow\left|p_{x}\right| n_{q h} \uparrow\right)\right|^{2}+\left|\left(n_{e} \downarrow\left|p_{x}\right| n_{q h} \uparrow\right)\right|^{2}\right] \delta_{\mathbf{k}_{e} \mathbf{k}_{h}}
\end{aligned}
$$

Dessa maneira podemos ver claramente que a expressão de luminescência da forma como foi desenvolvida até aqui é diagonal em $\mathbf{k}_{e}$ e $\mathbf{k}_{h}$. Essa expressão é válida, portanto, para transições diretas. Em sistemas $\delta$-doping tipo p porém, o espalhamento dos buracos por flutuações de curto alcance do potencial das impurezas quebra a regra de conservação do momento k. Resultados experimentais como os de Richards et al. ${ }^{[28]}$, que mostram na fotoluminescência de estruturas $\delta$-doping tipo p o 'Fermi Edge Enhancement', um fenômeno associado a transições indiretas, sustentam esta hipótese.

Devemos então obter uma expressão para a força de oscilador que leve em conta transições indiretas ao invés de transições diretas. Como as flutuações de curto alcance do potencial quebram a regra de conservação de $\mathbf{k}$, fazemos o mesmo substituindo o termo $\delta_{\mathbf{k}_{\mathbf{e}} \mathbf{k}_{h}}$ por 1 na expressão da força de oscilador (4.18). Dessa forma encontramos a seguinte expressão para a força de oscilador para transições indiretas:

$$
\begin{aligned}
& f_{n_{e} n_{g h}}\left(\mathbf{k}_{e}, \mathbf{k}_{h}\right)= \\
& \quad \frac{4}{m_{0}\left[E_{n_{e}}\left(\mathbf{k}_{e}\right)-E_{n_{q h}}\left(\mathbf{k}_{h}\right)\right]}\left[\left|\left(n_{e} \uparrow\left|p_{x}\right| n_{q h} \uparrow\right)\right|^{2}+\left|\left(n_{e} \downarrow\left|p_{x}\right| n_{q h} \uparrow\right)\right|^{2}\right]
\end{aligned}
$$

\subsection{Probabilidades de ocupação}

Para se calcular o espectro de luminescência através da expressão (4.4) é necessário determinar-se a função de ocupação dos estados dos por- 
tadores majoritários e minoritários. Estas duas representam situações completamente diferentes.

A $\mathrm{T}=0 \mathrm{~K}$ a ocupação dos portadores majoritários é bem definida, se o estado se encontra abaixo do nível de Fermi do sistema, ele está ocupado, se ele se encontra acima deste, está vazio. A uma temperatura finita a ocupação destes estados é descrita pela função de distribuição de Fermi.

Para os portadores minoritários a situação é diferente. A $\mathrm{T}=0 \mathrm{~K}$ e sem iluminação não há, em um sistema sem outras impurezas além do plano de dopagem, esse tipo de portadores. Quando o sistema é submetido a iluminação, portadores que estão em estados abaixo do nível de Fermi são promovidos à banda de portadores majoritários e permanecem lá por um tempo de recombinação médio. Num sistema a temperatura finita, este tempo médio de recombinação é suficiente para que os estados promovidos à banda de portadores majoritários decaiam para o estado fundamental dela. Desta maneira, a distribuição de estados de portadores minoritários se dará em torno deste estado fundamental, com uma certa dispersão devido à temperatura finita. Para descrever a ocupação destes estados usaremos a função de distribuição de Boltzmann em torno do estado fundamental da banda.

Aplicando a sistemas $\delta$-doping tipo $\mathrm{p}$, obtemos como função probabilidade de ocupação para buracos $N_{n_{q h} \mathrm{k}_{h}}$ :

$$
\left[1-N_{\left.n_{q h} \mathbf{k}_{h}\right]}\right]=\frac{1}{e^{\left[E_{F}-E_{n_{q h}}\left(\mathbf{k}_{h}\right)\right] / k T}}
$$

e para elétrons $N_{n_{e} k_{e}}$ :

$$
N_{n_{e} \mathbf{k}_{e}}=e^{-\left[E_{n_{e}}\left(\mathbf{k}_{e}\right)-E_{F_{n}}\right] / k T},
$$

onde $E_{F_{n}}$ é o quase-nível de Fermi para os elétrons fotocriados. 
Em sistemas $\delta$-doping tipo n, teremos, para elétrons $N_{n_{\mathrm{e}} k_{e}}$ :

$$
N_{n_{e} \mathbf{k}_{e}}=\frac{1}{e^{\left[E_{F}-E_{n_{e}}\left(\mathbf{k}_{e}\right)\right] / k T}}
$$

e para buracos $N_{n_{q h} \mathbf{k}_{h}}$ :

$$
\left[1-N_{n_{q h} \mathbf{k}_{h}}\right]=e^{\left[E_{n_{q h}}\left(\mathbf{k}_{h}\right)+E_{F_{p}}\right] / k T}
$$

onde $E_{F_{p}}$ é o quase-nível de Fermi para os buracos fotocriados.

\subsection{Dependência do 'gap' com a tempe- ratura}

Como o valor do 'gap' de energia varia com a temperatura. é necessário para que possamos comparar nossos espectros com espectros experimentais que incluamos essa variação no cálculo do espectro de luminescência. Como o 'gap' do GaAs é de $1519,2 \mathrm{meV}$ e para temperaturas de $300 \mathrm{~K}$ essa variação é de aproximadamente $100 \mathrm{meV}$, ou seja, cerca de $6 \%$ do valor do mesmo, é razoável sua inclusão após o cálculo concluído. Dessa maneira não será necessário recalcular-se a estrutura de bandas para cada temperatura em que se calcula o espectro.

Utilizamos a fórmula de Varshni ${ }^{[66]}$ para o cálculo dessa variação:

$$
E_{g}(T)=\left(1519,2-\frac{0,5405 T^{2}}{204+T}\right) m e V \quad(T \text { em } K)
$$




\subsection{Programa de cálculo da luminescência}

De posse das expressōes necessárias podemos então calcular teoricamente o espectro de luminescência. Tendo utilizado o programa autoconsistente, em sua versão com o Hamiltoniano de Kane, para calcular o potencial, os estados e seus autovalores e de posse do valor do nível de Fermi a $\mathrm{T}=0 \mathrm{~K}$, determinamos a ocupação de cada nível a diferentes temperaturas. Para os portadores majoritários utilizamos a distribuição de Fermi e para os minoritários, a distribuição de Boltzmann. Note-se que todos os portadores majoritários devem ser utilizados aqui, não só os que se encontram abaixo da energia de Fermi. Estados acima do nível de Fermi a $\mathrm{T}=0 \mathrm{~K}$ têm diferentes valores de ocupação a temperaturas finitas que são calculadas através da distribuição estatística de Fermi.

Calcula-se então a integral de superposição entre os estados das bandas de portadores minoritários e majoritários, multiplicando-se esse valor pelas ocupações de cada um desses estados à temperatura desejada. Calcula-se também a diferença de energia entre esses estados. Esse valor será a posição nominal do pico, o qual será descrito por uma Lorentziana com o alargamento de banda sugerido ad hoc. Nesse ponto, faz-se a distinção entre os cálculos que levam em conta apenas as transições diretas e os que levam em conta transições diretas e indiretas. Para transições diretas calcula-se a integral de superposição apenas dos estados de mesmo vetor de onda $\mathbf{k}$, enquanto que para o outro caso, a integral é calculada para quaisquer estados indiferentemente do valor de $\mathbf{k}$.

Após subtrair-se o valor da redução do 'gap' com a temperatura do valor nominal do pico associado a cada Lorentziana calculada, devemos somar todas as Lorentzianas referentes a cada temperatura numa só curva para obter-se o espectro correspondente a esta temperatura. O programa calcula espectros para cinco temperaturas diferentes em uma única submissão. 


\section{Capítulo 5}

\section{Estrutura de Bandas}

Este capítulo apresenta os resultados obtidos com o programa do cálculo de estrutura de bandas. Apresentaremos em primeiro lugar os testes executados a fim de determinar a correção do programa. Em seguida virão os potenciais, as estruturas de minibandas e sub-bandas, tanto para poços isolados como para super-redes. Para descrever poços isolados utilizamos super-redes com período $\mathrm{d}=500 \AA$, acima do limite em que os estados de um poço interagem com os de seu vizinho. Super-redes de período $\mathrm{d}=200 \AA$ também são estudadas. Por comodidade escolhemos trabalhar apenas com três concentrações de carga diferentes. Os valores utilizados correspondem a concentrações usualmente consideradas, no caso de poços $\delta$-doping tipo p, como baixa $\left(N_{s}=3 \times 10^{12} \mathrm{~cm}^{-2}\right)$, média $\left(N_{s}=8 \times 10^{12} \mathrm{~cm}^{-2}\right)$ e alta $\left(N_{s}=3 \times 10^{13} \mathrm{~cm}^{-2}\right)$.

Neste capítulo, faremos ainda um estudo comparativo entre poços tipo p e tipo n. Na comparação entre os resultados obtidos para poços $\delta$ doping tipo $\mathrm{p}$ com os obtidos para poços tipo $\mathrm{n}$ haverá a única exceção feita à regra de concentrações: para um poço tipo $n$, a concentração de $3 \times 10^{13} \mathrm{~cm}^{-2}$ é considerada muito alta e por este motivo muito difícil de ser observada. Nesse estudo portanto, utilizaremos a concentração de $1,5 \times 10^{13} \mathrm{~cm}^{-2}$ ao invés de $3 \times 10^{13} \mathrm{~cm}^{-2}$. 
Os poços estudados têm um perfil de dopagem Gaussiano, com $\sigma=10 \AA$, que corresponde a uma largura a meia altura $\Delta z=23.55 \AA$. Apresentaremos também um estudo da variação dos potenciais dos poços, do nível de Fermi e dos estados ocupados em função do alargamento da distribuição dos dopantes na camada $\delta$.

\subsection{Testes de adequação}

Os testes de adequação do programa de cálculo de bandas de estruturas $\delta$-doping tipo p serão apresentados a seguir. Três testes diferentes foram feitos. Os dois primeiros utilizaram o método aqui desenvolvido para calcular sistemas baseados em heteroestruturas composicionais (poços quadrados): o primeiro determinou o número necessário de ondas planas a ser utilizado para o cálculo desse tipo de heteroestruturas e o segundo a precisão de cálculo de nossa matriz $\mathbf{k} \cdot \mathbf{p}$. O terceiro teste serviu para determinar a qualidade de nosso procedimento autoconsistente, utilizando para tanto resultados de cálculos de estrutura de bandas de poços $\delta$-doping tipo $n$.

\subsubsection{Poços quadrados e super-redes baseadas em heteroestruturas $G a A s / G a A l A s$}

O programa desenvolvido neste trabalho pode ser utilizado para o cálculo de uma gama de sistemas maior do que inicialmente previsto. Usando essa qualidade foi possível fazer testes comparativos de sua performance com resultados da literatura, em casos bem conhecidos. Um desses casos é o de poços de potencial quadrados baseados em heteroestruturas $\mathrm{GaAs} / \mathrm{GaAlAs}$. Como tais sistemas apresentam duas regiões distintas, com composições também distintas e, em decorrência deste fato, características físicas diferentes, é necessário fazer uma nova aproximação.

Consideraremos aqui que toda a estrutura tenha uma mesma constante 
dielétrica, a constante do material da região do poço, sendo as barreiras determinadas por um potencial quadrado inserido ad hoc, com uma altura de barreira determinada pela diferença de potencial criada na interface entre os dois materiais. Esta aproximação não deve causar complicações adicionais visto que os portadores não penetram substancialmente na barreira e, portanto, seus estados não dependem da constante dielétrica da barreira. Além do cálculo ser factível, existe uma segunda qualidade desses sistemas que nos é adequada. Se a concentração de portadores do sistema for pequena não é necessário calculá-lo autoconsistentemente. Isto decorre do fato de o potencial determinado pela carga dos portadores ser muito menor que o do poço em si, podendo portanto ser desprezado.

O potencial de um poço quadrado representa um potencial mais difícil de ser descrito por uma aproximação de ondas planas que um potencial do tipo $\delta$-doping. Isto se deve ao fato dele ter uma variação abrupta na borda do poço. Dessa maneira este potencial é o indicado para se testar a convergência no número de ondas planas necessária ao nosso cálculo.

A fig. 5.1 mostra a estrutura de bandas de um poço quadrado isolado não-dopado, calculada com diferentes números de ondas planas. Os parâmetros de Luttinger utilizados neste cálculo $\left(\gamma_{1}=6.85, \gamma_{2}=2.1\right.$ e $\gamma_{3}=2.9$ ) foram tirados da ref. [63]. Os resultados diferem grandemente entre os cálculos com 21 e 41 ondas planas. Para um maior número de ondas planas porém, os resultados não diferem de maneira significativa. A repulsão entre as sub-bandas conhecida dos cálculos de poços baseados em heteroestruturas fica claramente definida para as estruturas calculadas com 41 ondas planas ou mais.

Determinado o número necessário de ondas planas podemos calcular sistemas comparáveis aos da literatura. A ref. [41] apresenta resultados de um cálculo de estrutura da banda de valência para um poço quadrado de $G a A s / G a_{x} A l_{1-x} A s$ com x=0,51 e período de $112 \AA$. Os parâmetros de Luttinger são os mesmos que utilizamos anteriormente em nosso cálculo $\left(\gamma_{1}=6,85, \gamma_{2}=2,1\right.$ e $\left.\gamma_{3}=2,9\right)$. Para calcular a descontinuidade da banda de valência na interface, os autores utilizaram 
101 O.P. $\quad 61$ O.P. $\quad$ 41 O.P. $\quad 21$ O.P.

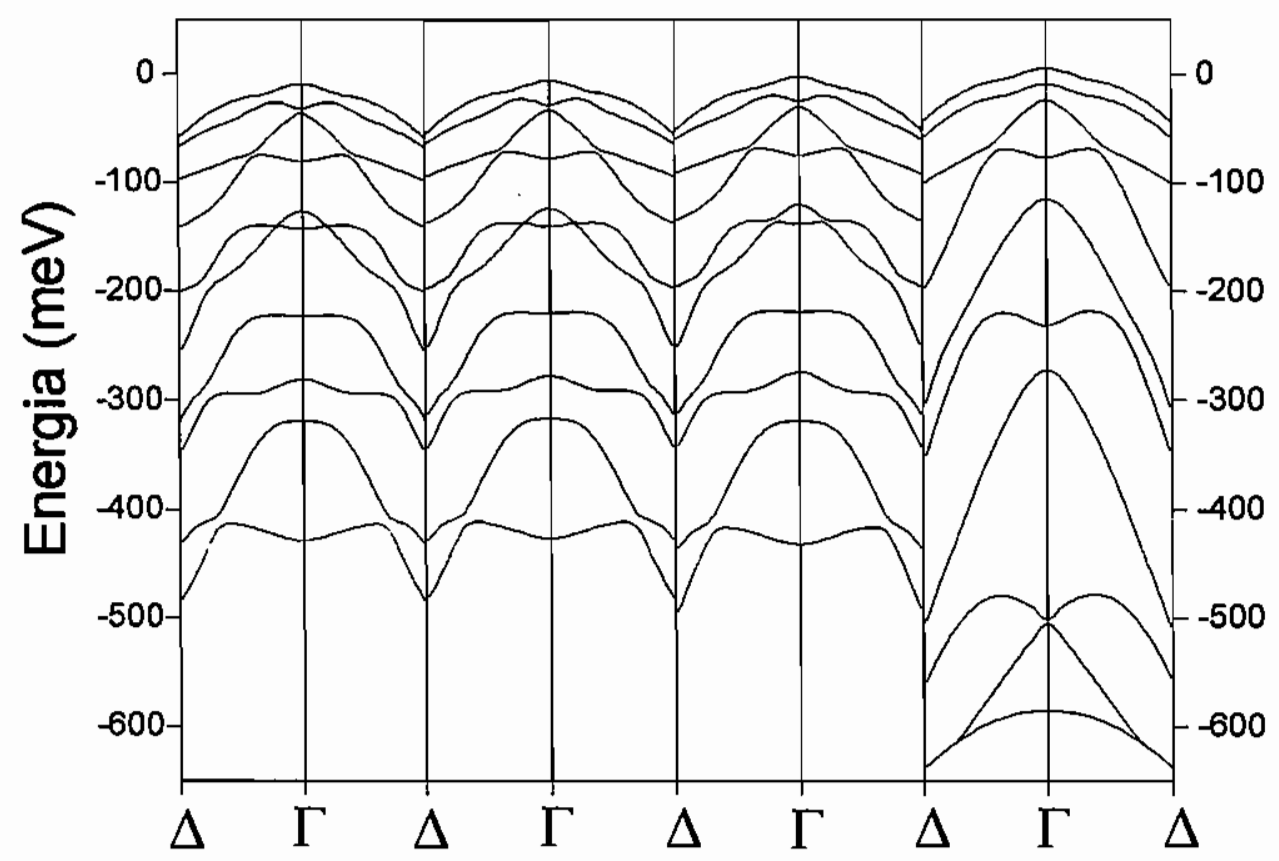

Figura 5.1: Estrutura de bandas para um poço quadrado não-dopado e isolado com largura $97 \AA$, e altura de barreira de $300 \mathrm{meV}$. O número de ondas planas usadas na descrição do potencial do poço varia de 21 a 101.

para estimar o 'gap', a expressão:

$$
E_{G}=E_{G}(G a A s)+1,04 x+0,47 x^{2} .
$$

Para determinar a fração da descontinuidade do 'gap' na banda de valência foi usada a regra de $43 \%^{[67]}$. Calculando a altura da barreira encontramos o valor de $280 \mathrm{meV}$. A fig. 5.2 apresenta uma comparação entre um cálculo executado por nosso programa para um sistema rigorosamente igual ao de Altarelli et al. ${ }^{[41\}} \mathrm{com}$ o resultado obtido por eles no referido artigo.

É necessário salientar que esses dois cálculos foram feitos com sistemas fundamentalmente diferentes: nosso sistema simula uma heteroestrutura, criada por uma perturbação sobre um material homogêneo, isto 


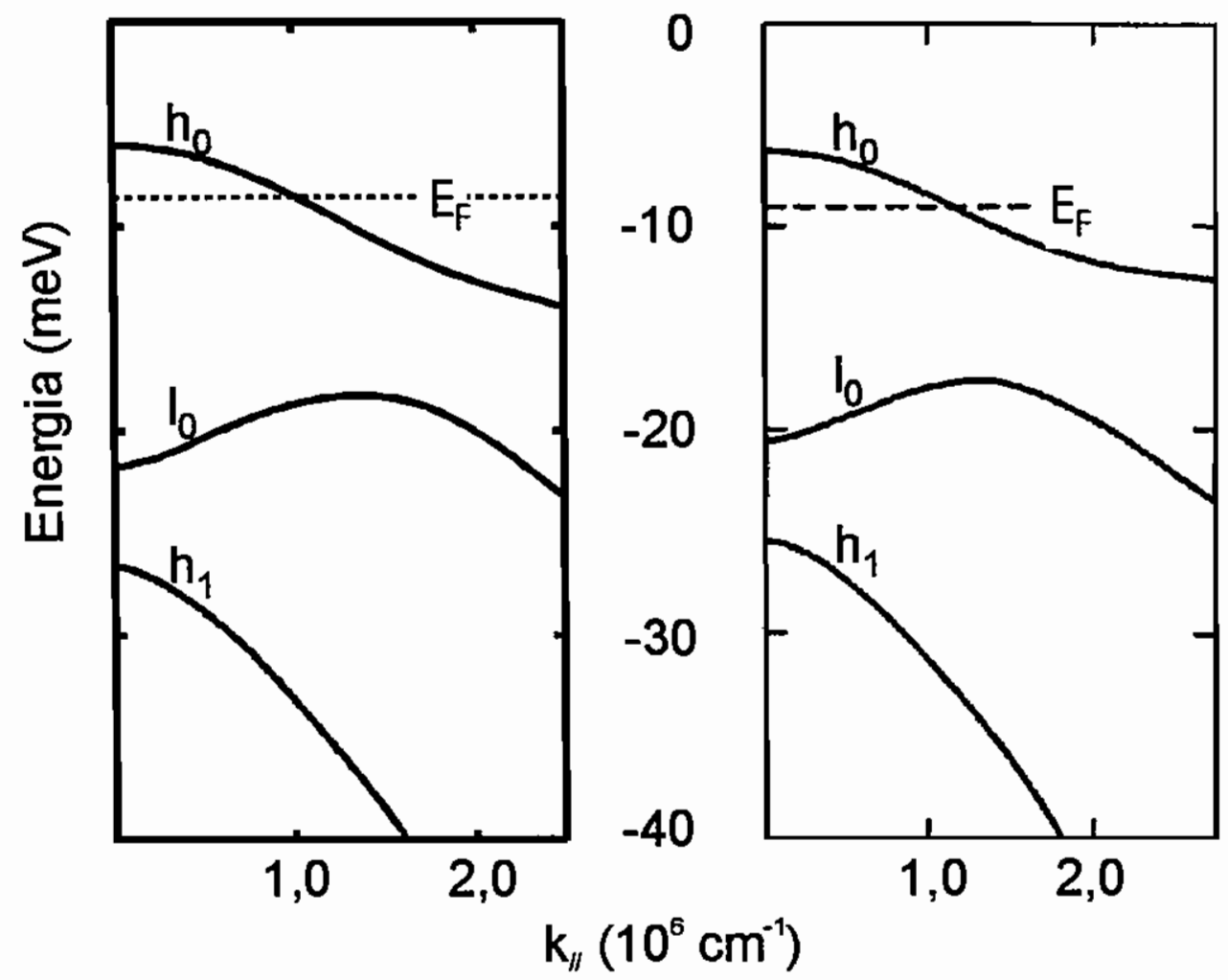

Figura 5.2: Estrutura de sub-bandas na direção perpendicular à direção de crescimento para um poço quadrado isolado de largura $112 \AA$, altura de

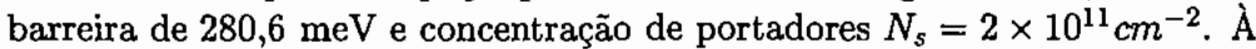
esquerda nossos resultados e à direita, para comparação, a fig. 5 do trabalho de Altarelli et al. ${ }^{[41]}$

é, impomos um potencial ad hoc sobre GaAs volumétrico e Altarelli et al. estudam uma heteroestrutura criada por regiões com composições químicas diferentes. A concordância entre os resultados é excelente, sendo evidenciado o espalhamento entre bandas diferentes.

\subsubsection{Poços $\delta$-doping tipo $n$}

O cálculo da estrutura de bandas de poços $\delta$-doping tipo p em $G a A s$ apresenta dificuldades devido à presença de dois tipos diferentes de 
portadores numa mesma região do espaço. Para poços $\delta$-doping tipo n a tarefa de calcular sua estrutura de bandas é muito mais simples. Continua havendo necessidade de se fazer cálculos autoconsistentes, porém, devido à existência de um único tipo de portador, a equação da massa efetiva pode ser resolvida no espaço direto através de integrais. Isso diminui drasticamente as necessidades computacionais. Cálculos de estruturas de bandas de poços tipo n estão presentes na literatura desde o começo desta década ${ }^{[8,11,20,68]}$. O estudo sistemático de tais heteroestruturas vem sendo feito pelo grupo associado ao Laboratório de Novos Materiais Semicondutores do Instituto de Física da USP tanto do ponto de vista teórico ${ }^{[17,21,22,69-72]}$ e experimental ${ }^{[9,15,17,73-75]}$. Dessa maneira dispomos de um método para o cálculo de estrutura de bandas de super-redes $\delta$-doping tipo n desenvolvido no espaço direto.

O método para o cálculo da estrutura de bandas de energia para poços $\delta$-doping tipo p pode ser facilmente utilizado para o cálculo da estrutura de bandas de poços- $\delta$ tipo $n$. Para tanto basta utilizar a matriz de Kane, substituir o potencial dos aceitadores por um potencial de doadores $\mathrm{e}$ calcular o nível de Fermi dos elétrons na banda de condução ao invés do de buracos na banda de valência. Na prática, quanto ao potencial é necessário apenas trocar o sinal. Já com relação ao nível de Fermi, deve-se contar os portadores na banda de condução preenchendo os estados na direção inversa a que se contava anteriormente.

Este procedimento demanda uma capacidade computacional desnecessária em se tratando de calcular apenas a estrutura da banda de condução. Para simplificar podemos calcular a mesma estrutura com um sistema em que a matriz de Kane é substituída por uma matriz diagonal $2 \times 2$ :

$$
H_{\text {ele }}=\left[\begin{array}{cc}
A & 0 \\
0 & A
\end{array}\right]
$$

onde A representa os estados eletrônicos e é descrito pela expressão 


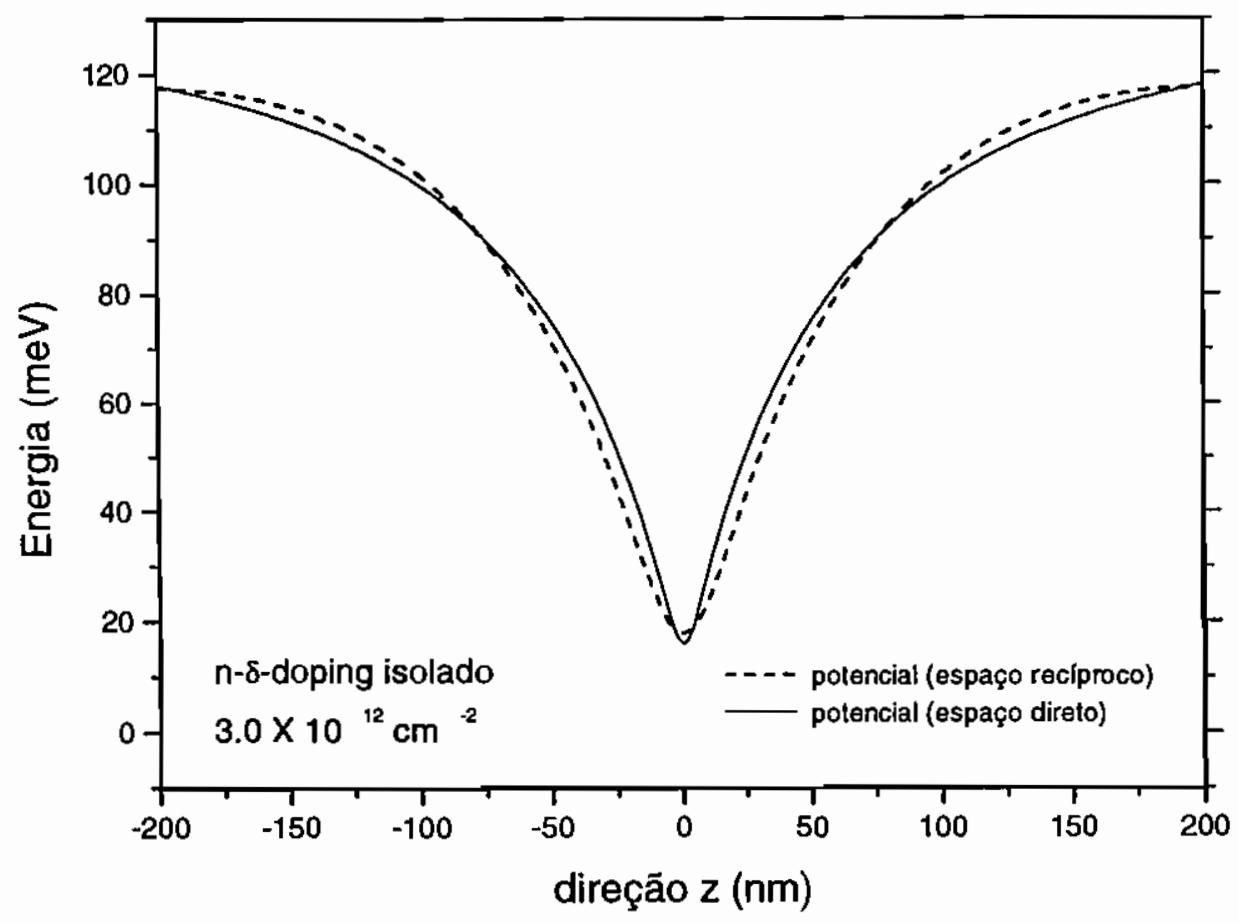

Figura 5.3: Perfis de potencial para um poço $\delta$-doping tipo $n$ calculados com os dois diferentes métodos.

$$
A=\frac{\hbar^{2}}{2 m^{*}}\left[k_{x}^{2}+k_{y}^{2}+k_{z}^{2}\right]
$$

A existência de um método desenvolvido e testado (que se utiliza do espaço direto) para o cálculo da estrutura da banda de condução em poços $\delta$-doping tipo $\mathrm{n}$ nos possibilita testar o procedimento de autoconvergência de nosso programa de cálculo. Para tanto, basta calcular o mesmo sistema pelos dois métodos e comparar os potenciais convergidos.

A fig. 5.3 apresenta dois cálculos feitos para sistemas com mesma concentração $\left(N_{s}=8 \times 10^{12} \mathrm{~cm}^{-2}\right)$ valendo-se dos dois diferentes métodos. Com o método do espaço direto foi calculado um poço $\delta$-doping tipo $\mathrm{n}$ isolado ${ }^{(76)}$, com perfil de dopagem quadrado e com o método aqui desenvolvido, uma super-rede de poços $\delta$-doping tipo $n$ com perfil de 
dopagem Gaussiano e largura de super-rede $d=400 \AA$. Apesar das diferenças entre os sistemas, mais uma vez os resultados apresentados têm uma ótima concordância.

\section{$5.2 \quad$ Estruturas $\delta$-doping tipo $\mathbf{p}$}

Os resultados dos cálculos de estrutura de bandas de super-redes $\delta$ doping tipo p serão mostrados a seguir. Primeiro apresentaremos os potenciais relacionados aos sistemas estudados: potenciais coulombianos autoconsistentes e potenciais totais incluindo além do anterior o termo de troca e correlação. Em seguida apresentaremos resultados dos cálculos da estrutura de bandas incluindo um estudo para determinar a importância da dispersão dos dopantes. Todos os resultados apresentados nesta seção foram obtidos com o programa de cálculo autoconsistente, utilizando sempre 20 ondas planas na base de expansão do potencial.

\subsubsection{Potenciais}

A fig. 5.4 apresenta potenciais para as concentrações alta e baixa de dopantes $\left(N_{s}=3 \times 10^{13} \mathrm{~cm}^{-2}\right.$ e $N_{s}=3 \times 10^{12} \mathrm{~cm}^{-2}$, respectivamente). Apenas os potenciais de sistemas com períodos $d=500 \AA$ são mostrados, pois a conformação e as profundidades dos potenciais de sistemas com $d=200 \AA$ diferem muito pouco das dos primeiros. As linhas pontilhadas apresentam o potencial coulombiano, sem troca e correlação. As linhas sólidas apresentam o potencial total, incluindo troca e correlação. Duas curvas são necessárias em virtude dos potenciais de troca e correlação serem diferentes para cada tipo de portador, como foi salientado no capítulo 3. Ao observarmos as expressões (3.52) a (3.55), percebemos que no limite de $\mathbf{k}$ tendendo ao ponto $\Gamma$, a matriz $\left(m \mathbf{k} K\left|V_{X C}\right| m^{\prime} \mathbf{k} K^{\prime}\right)$ da eq. (3.51) é diagonal e os valores de seus elementos são $\left(K\left|Q_{X C}\right| K^{\prime}\right)=\left(K\left|V_{X C \frac{3}{2}}\right| K^{\prime}\right)$ e $\left(K\left|T_{X C}\right| K^{\prime}\right)=\left(K\left|V_{X C \frac{1}{2}}\right| K^{\prime}\right)$. Dessa maneira podemos, ao menos para o ponto $\Gamma$, definir univoca- 
mente os potenciais de troca e correlação de buracos leves e pesados movimentando-se ao longo da direção $z$ (indicados na fig. 5.4 como lh e hh, respectivamente).

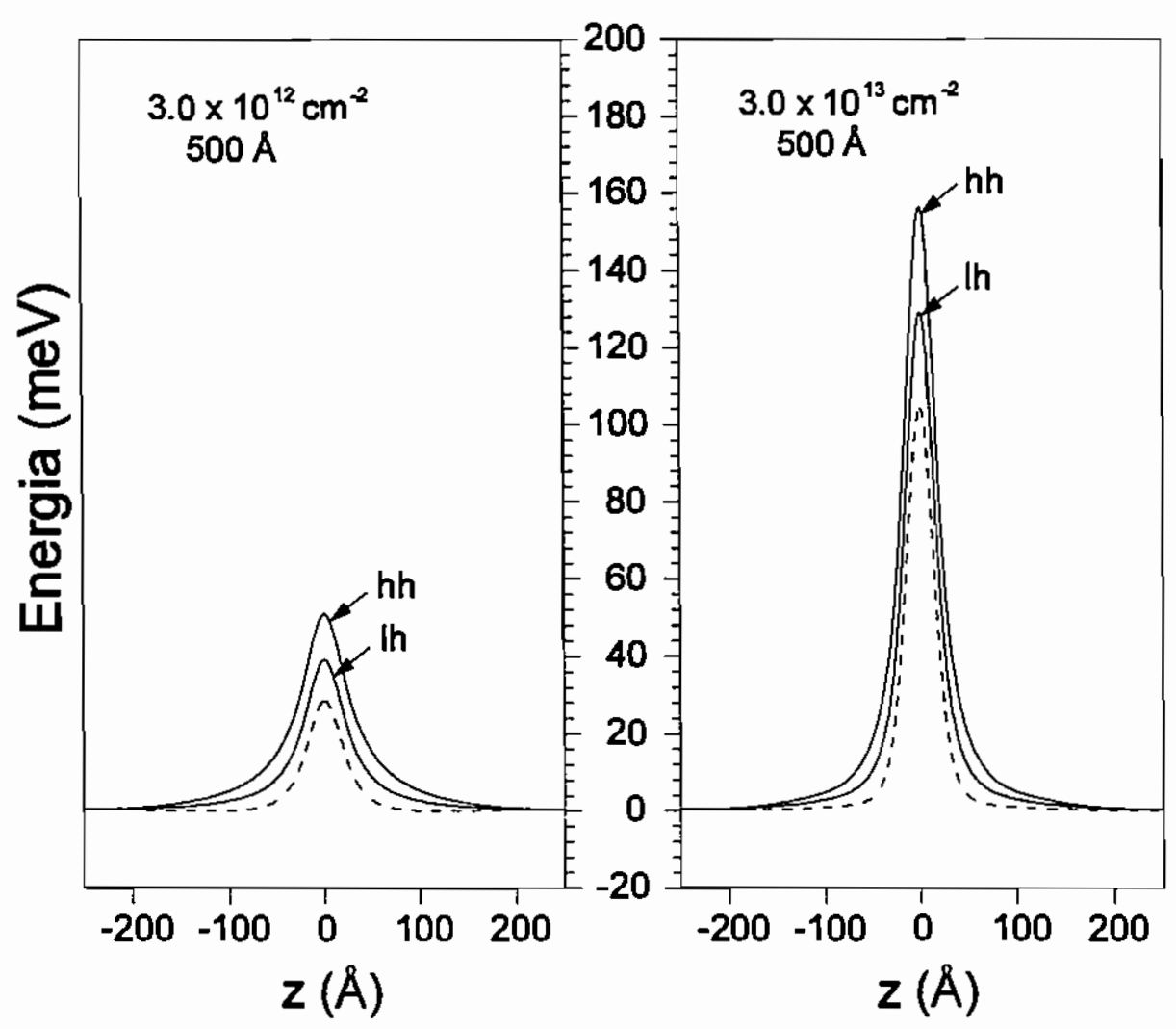

Figura 5.4: Perfis de potencial para buracos para super-redes $\delta$-doping tipo p com período $d=500 \AA$, concentração de dopantes $N_{s}=3 \times 10^{12} \mathrm{~cm}^{-2}$ (esquerda) e $N_{s}=3 \times 10^{13} \mathrm{~cm}^{-2}$ (direita), e $\sigma=10 \AA$. As linhas tracejadas indicam o potencial coulombiano e as sólidas o potencial total incluindo troca e correlação. O zero de energia foi fixado como o topo da barreira do potencial coulombiano para buracos.

Os potenciais de buracos pesados são sempre mais profundos que os de buracos leves pois o potencial de troca e correlação é atrativo e cresce monotonicamente com a densidade, e porque a densidade de buracos pesados é sempre maior que a de buracos leves. No total o efeito do potencial de troca e correlação é relativamente grande nas super-redes 


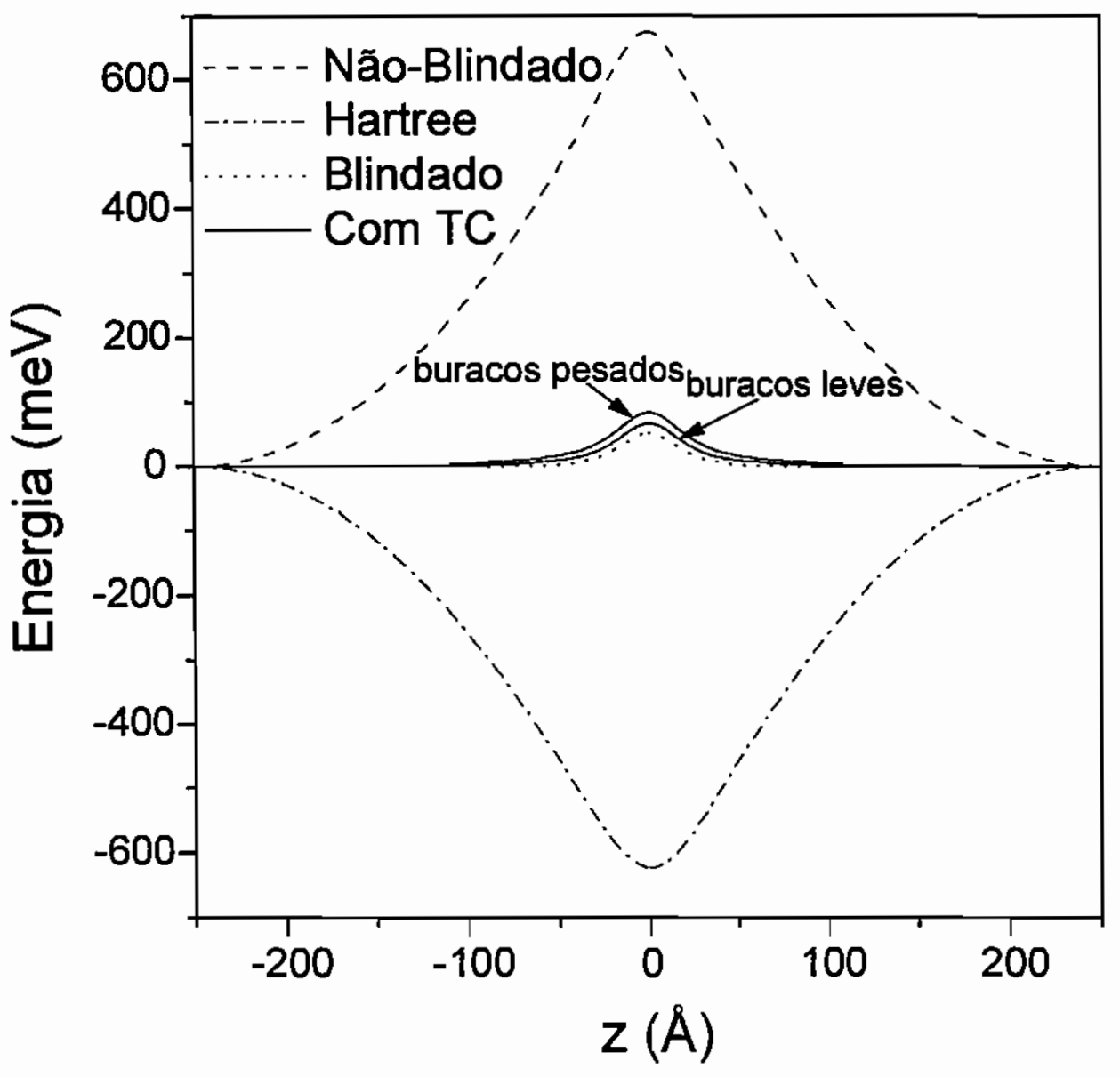

Figura 5.5: Potenciais total e parcial para buracos numa super-rede $\delta$ doping tipo $p$ de período $500 \AA$. O potencial não-blindado é devido à camada de aceitadores ionizados, negativamente carregada, envolta por um gás de buracos homogeneamente distribuídos de maneira a neutralizar a carga total. O potencial de Hartree é devido à distribuição de buracos calculada autoconsistentemente envolta por uma distribuição de cargas negativas homogênea de forma a, uma vez mais, neutralizar a carga total. O potencial blindado é o potencial de Coulomb total, como é visto pelos buracos. Ele é a soma dos dois potenciais anteriores. Os potenciais indicados como de buracos leves e pesados são a soma do potencial coulombiano total com os potenciais de troca e correlação (TC) de buracos leves e pesados, respectivamente. 
$\delta$-doping estudadas. Aqui a contribuição da troca e correlação ao potencial total mantém-se entre $30 \%$ para as concentrações mais altas e $40 \%$ para as mais baixas. Essas contribuições não parecerão tão altas se compararmos o potencial de troca e correlação com o potencial de Hartree, sem a blindagem causada pelo potencial da camada de aceitadores negativamente carregada (ver fig. 5.5). Esta última forma, um poço profundo que é quase completamente blindado pelo potencial de Hartree dos buracos. Quando comparado ao potencial de Hartree, pode-se ver que o potencial de troca e correlação é da ordem de apenas $5 \%$ para buracos pesados e $3 \%$ para buracos leves. A profundidade total dos poços aumenta com o aumento da concentração. Nas superredes de $500 \AA$ os poços para buracos pesados para as concentrações mencionadas acima têm profundidade de $48 \mathrm{meV}, 85 \mathrm{meV}$ e $148 \mathrm{meV}$, respectivamente. Para as super-redes de $200 \AA$, eles têm valores ligeiramente menores. Devido à menor distância entre os planos de aceitadores, as distribuições dos buracos em cada célula ultrapassam os seus limites, gerando um fundo de cargas positivas que blinda o potencial da camada de aceitadores mais efetivamente. De maneira geral, os poços $\delta$-doping tipo p de uma determinada concentração nominal são mais rasos que os poços $\delta$-doping tipo n com a mesma concentração [21].

\subsubsection{Estrutura de bandas}

Na fig. 5.6 são mostradas as estruturas de bandas para as super-redes calculadas. Cada sub-figura corresponde a uma dada concentração, e mostra, em seu lado esquerdo, a super-rede com período de $500 \AA \mathrm{e}$, em seu lado direito, a super-rede com período de $200 \AA$. São mostradas as minibandas perpendiculares ao plano de dopantes entre o centro da $1^{\underline{a}}$ Zona de Brillouin, em $\Gamma$ e seu limite em $Z$, assim como as subbandas ao longo das direções $\Delta$ e $\Delta^{\prime}$, paralelas ao plano de dopantes. A escala para os vetores de onda é a mesma em cada figura. Elas são determinadas pela distância $\Gamma-Z$, que é de $\pi / d$.

Examinemos agora os sistemas com concentração de impurezas de $3,0 \times 10^{12} \mathrm{~cm}^{-2}$. Para a super-rede de $500 \AA$ temos uma primeira 

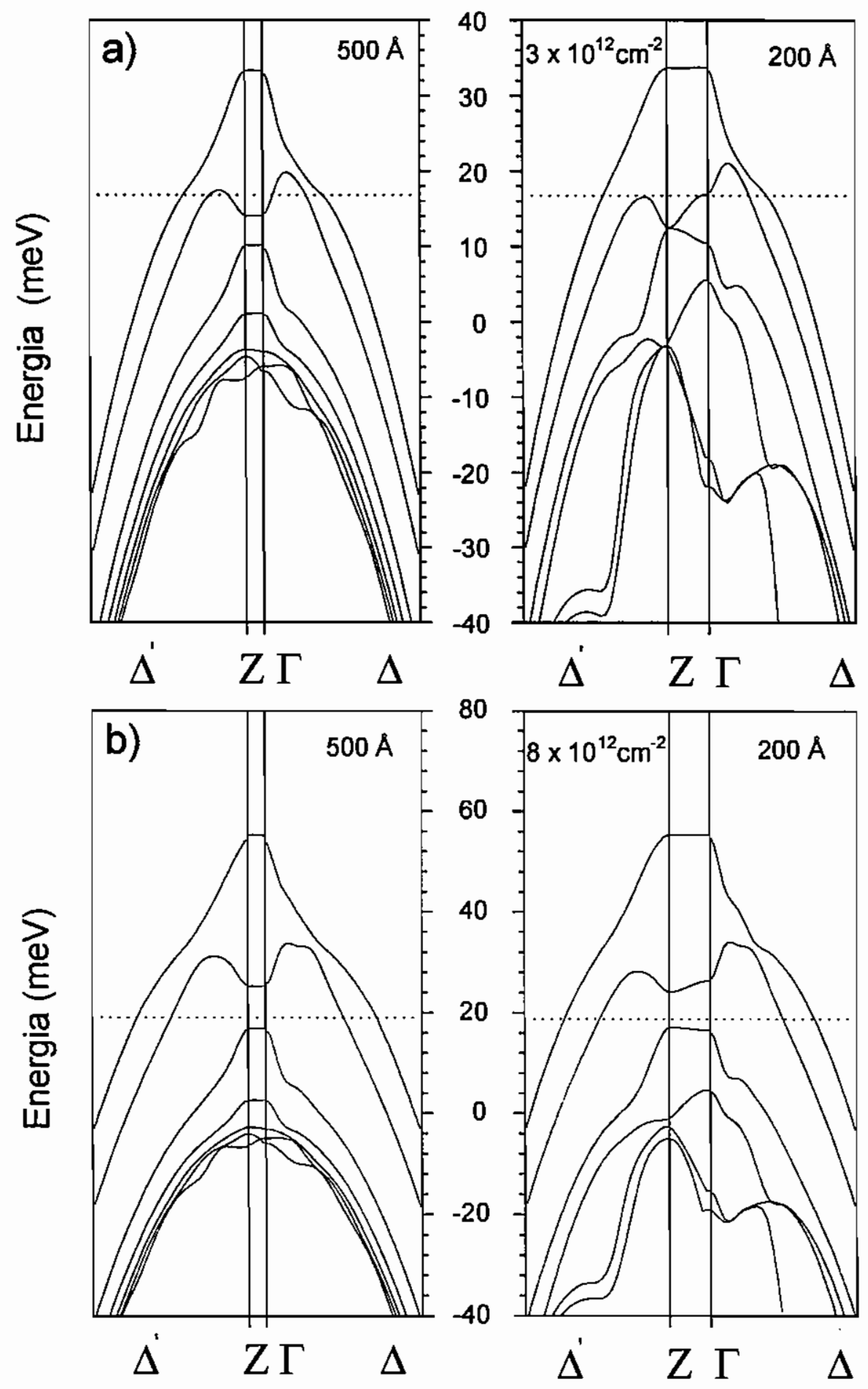


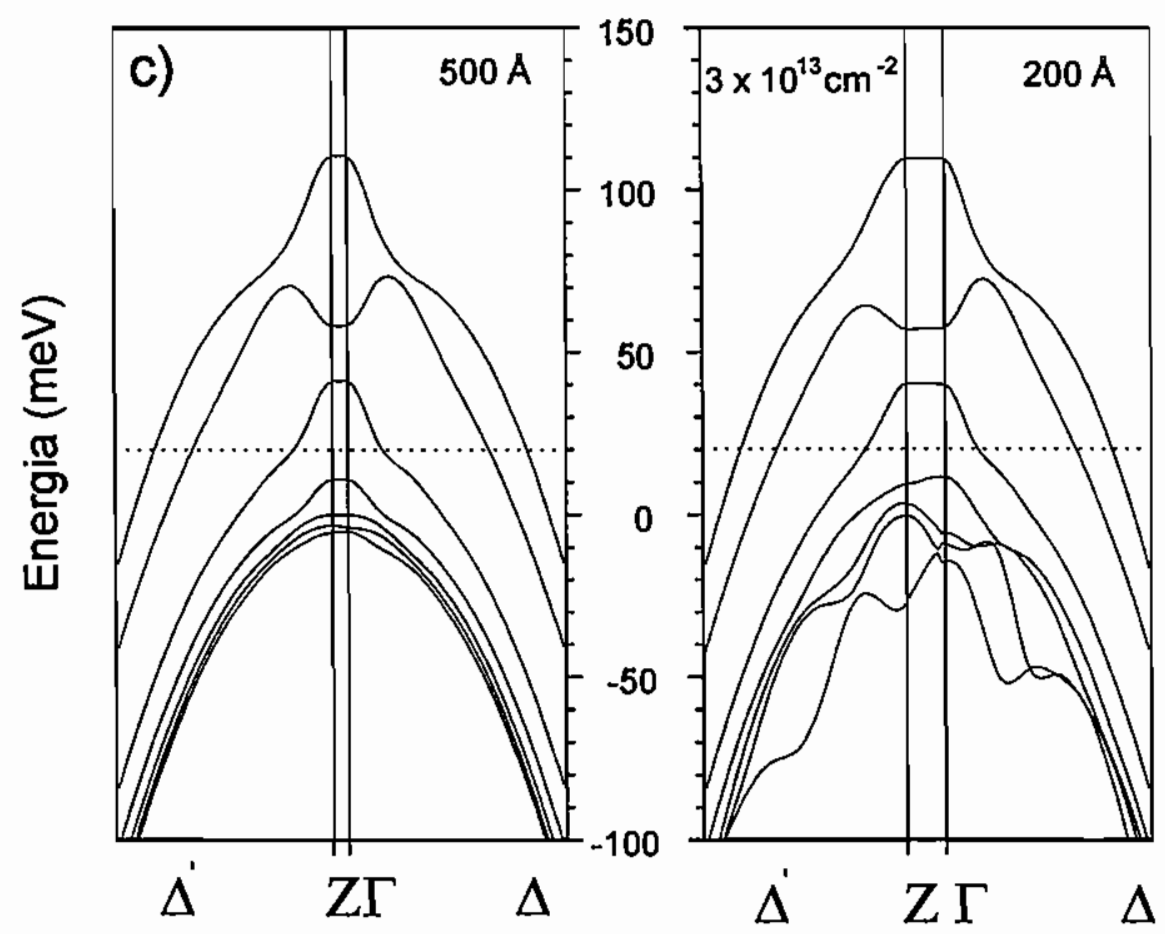

Figura 5.6: Estruturas de bandas (linhas sólidas) e níveis de Fermi (pontilhado) para super-redes $\delta$-doping tipo p com três diferentes concentraçōes de aceitadores $\left(3 \times 10^{12} \mathrm{~cm}^{-2}\right.$ para o sistema a), $8 \times 10^{12} \mathrm{~cm}^{-2}$ para o sistema b), mostrados na página anterior, e $3 \times 10^{13} \mathrm{~cm}^{-2}$ para o sistema c) ) e $\sigma=10 \AA$, períodos $500 \AA$ (esquerda) e $200 \AA$ (direita). A escala horizontal é ajustada pela distância $\Gamma$-Z. O zero de energia é ajustado para o topo da barreira, como mostra a fig. 5.4 .

minibanda de buracos pesados $\left(h h_{1}\right)$ sem dispersão $33 \mathrm{meV}$ abaixo da barreira de potencial comum a buracos leves e pesados, seguida por uma segunda minibanda, dessa vez de buracos leves $\left(l h_{1}\right)$, a $14 \mathrm{meV}$ abaixo da mesma barreira. A segunda e a terceira minibandas de buracos pesados $\left(h h_{2}\right.$ e $\left.h h_{3}\right)$ ainda se encontram abaixo da barreira. Todas as outras minibandas encontram-se acima da barreira, apresentando uma dispersão considerável. Ao observar-se o comportamento das bandas nas direções perpendiculares à direção de crescimento da amostra: nota-se o espalhamento entre as bandas de buracos leves e pesados já observado anteriormente para poços quânticos baseados em heteroes- 
truturas ${ }^{[41]}$. Ao observar-se a posição do nível de Fermi, nota-se que a maioria dos estados ocupados pertence à primeira banda de buracos pesados, tendo a primeira banda de buracos leves apenas uma pequena ocupação.

Na super-rede de mesma concentração e com período de $200 \AA$, vê-se que uma forte dispersão aparece a partir da primeira minibanda de buracos leves $\left(l h_{1}\right)$. A primeira minibanda de buracos pesados não apresenta dispersão e ocorre à mesma energia da minibanda similar no sistema com $500 \AA$. Da mesma forma que o sistema anterior, a maioria dos estados ocupados pertence à primeira minibanda de buracos pesados, com uma pequena ocupação da primeira minibanda de buracos leves.

Passando-se à observação dos sistemas com concentração de $8,0 \times 10^{12} \mathrm{~cm}^{-2}$, percebe-se que o aumento da concentração na camada de dopantes aprofunda o poço e desvia as minibandas para energias mais altas. Nos dois casos (períodos de 200 e $500 \AA$ ), quatro minibandas aparecem abaixo da barreira. A primeira banda de buracos pesados não apresenta dispersão e aparece nos dois sistemas à mesma energia, $55 \mathrm{meV}$ abaixo da barreira. A segunda minibanda é claramente identificável como a primeira de buracos leves $\left(l h_{1}\right)$, e a terceira como a segunda minibanda de buracos pesados $\left(h h_{2}\right)$. A quarta minibanda deve ser identificada como a segunda de buracos leves pelo forte espalhamento que ocorre entre ela e a terceira minibanda nas direções perpendiculares à direção de crescimento. O nível de Fermi se encontra na segunda minibanda, isto é, na minibanda $l h_{1}$.

Observando-se os sistemas com concentração de impurezas de $3,0 \times 10^{13} \mathrm{~cm}^{-2}$, vemos que para ambos os períodos, a seqüência das minibandas é a mesma, $h h_{1}, l h_{1}, h h_{2}, h h_{3}$ e $l h_{2}$. As três primeiras praticamente não apresentam dispersão tendo energias muito próximas para ambos os sistemas, $110 \mathrm{meV}, 60 \mathrm{meV}$ e $40 \mathrm{meV}$, respectivamente. Só a partir da quarta minibanda $\left(h h_{3}\right)$, os dois sistemas começam a diferir. Para o sistema com período de $500 \AA$ esta minibanda não apresenta dispersão, enquanto que para o sistema com $200 \AA$, ela apresenta. $O$ espalhamento das bandas está presente em ambos os sistemas e o nível 

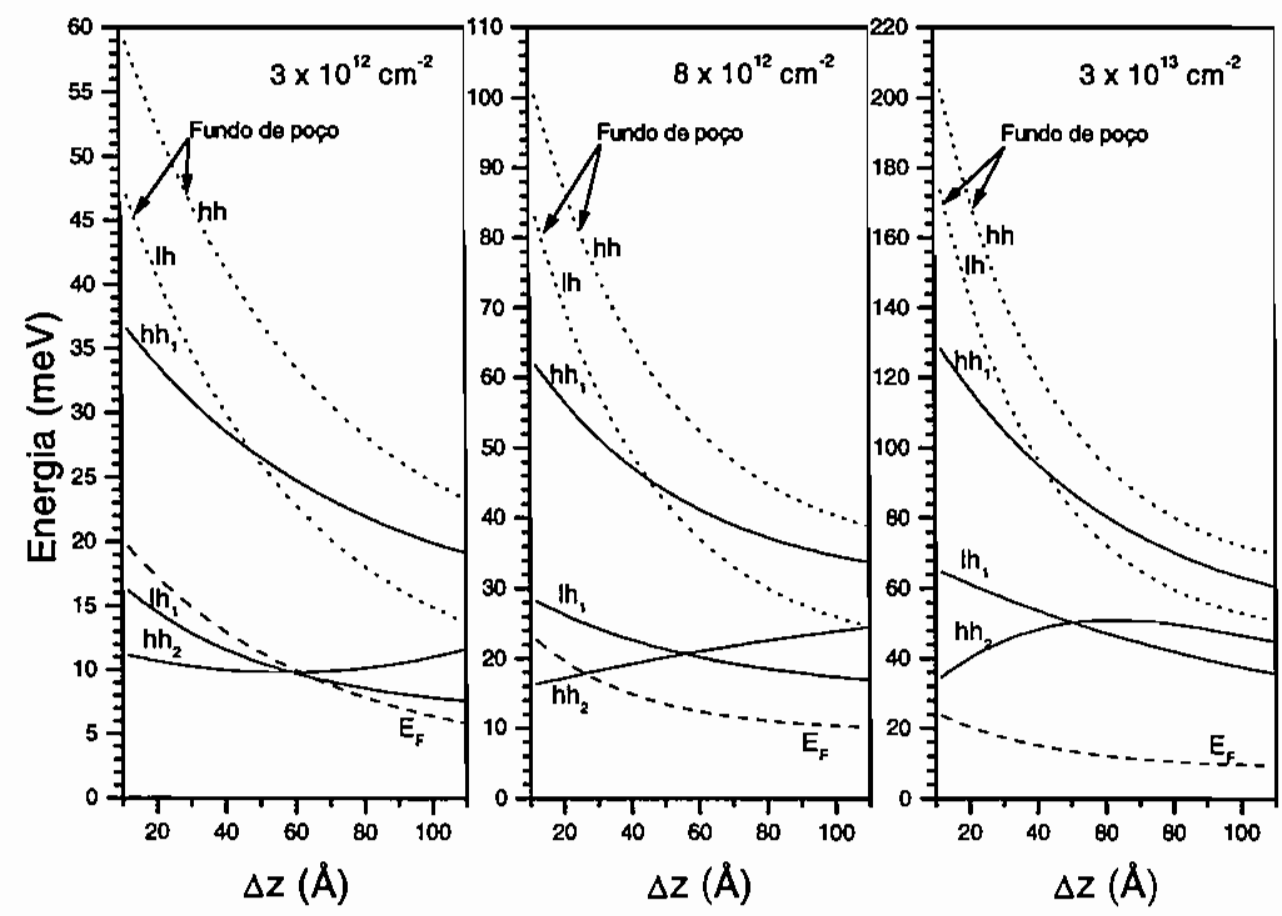

Figura 5.7: Fundos de poço (linhas pontilhadas), estados de buracos (linhas sólidas) e energias de Fermi (linhas tracejadas) para super-redes de poços $\delta$-doping tipo p com período de $500 \AA$, desenhadas em função do valor de $\Delta z$. São mostradas três diferentes concentrações: $N_{s}=3 \times 10^{12} \mathrm{~cm}^{-2}$, $N_{s}=8 \times 10^{12} \mathrm{~cm}^{-2}$ e $N_{s}=3 \times 10^{13} \mathrm{~cm}^{-2}$.

de Fermi se encontra na terceira sub-banda.

Até aqui tratamos de sistemas em que a dispersão da camada foi fixada em $\Delta z=23,55 \AA$. Valores experimentais de $\Delta z$, tirados da literatura $^{[28,33]}$ podem diferir de maneira significativa dos que utilizamos. Para estudar a variação dos resultados apresentados com a mudança desse valor, foram calculados sistemas nas três concentraçōes anteriores, para $\Delta z$ variando entre 12 e $120 \AA$. Os resultados são apresentados na fig. 5.7. Para uma dada concentração os poços se tornam mais rasos e o nível de Fermi sobe com o aumento de $\Delta z$, como era de se esperar. A mudança de posição dos estados de buracos com o aumento de $\Delta z$ é o resultado da competição de dois efeitos distintos: o aprofundamento do fundo do poço, que força os estados para baixo e o aumento do con- 
finamento, que os força para cima. Como pode ser visto na fig. 5.7, o afundamento do poço domina para os primeiros estados de buracos leves $\left(l h_{1}\right)$ ou pesados $\left(h h_{1}\right)$, enquanto que o segundo efeito é dominante no primeiro estado excitado de buracos pesados $\left(h h_{2}\right)$. Devido a esse fato, o ordenamento dos níveis muda com o aumento de $\Delta z$. Enquanto que para $\Delta z$ pequeno, o segundo estado de buracos pesados $\left(h h_{2}\right)$ fica acima do primeiro de buracos leves $\left(l h_{1}\right)$, para valores maiores de $\Delta z$, as posições se invertem. Com o aumento de $\Delta z$, o nível de Fermi dos sistemas adentra minibandas de índices mais altos. Podemos ilustrar com as super-redes de concentração mais baixa, onde o nível de Fermi, que ficava entre a primeira banda de buracos pesados $\left(h h_{1}\right)$ e a primeira banda de buracos leves $\left(l h_{1}\right)$, adentra a segunda banda de buracos pesados $\left(h h_{2}\right)$ e com as super-redes de concentração média, onde o nível estava entre o primeiro estado de buracos leves $\left(l h_{1}\right)$ e o segundo estado de buracos pesados $\left(h h_{2}\right)$ e também adentra o nível $\left(h h_{2}\right)$. Todos esses dados juntos demonstram que as estruturas eletrônicas de sistemas do $\delta$-doping tipo p são extremamente sensíveis a variações no perfil de dopagem.

\subsection{Comparação com resultados de outros autores para poços $\delta$-doping tipo $\mathrm{p}$ iso- lados}

Poços $\delta$-doping isolados em GaAs com uma concentração média de $\left(8 \times 10^{12} \mathrm{~cm}^{-2}\right)$ e com uma dispersão pequena em torno da camada $\delta$ $(\approx 20 \AA)$ são tratadas nas refs. [28] e [35] fazendo uso de métodos e aproximações distintos dos que usamos aqui. A fig. 5.8 apresenta para comparação, perfis de potencial para uma super-rede de período $d=500 \AA$, concentração de dopantes $N_{s}=8 \times 10^{12} \mathrm{~cm}^{-2} \mathrm{e}$ $\sigma=10 \AA$, calculados autoconsistentemente com nosso programa (a) e para poços isolados de mesma concentração, calculados por Reboredo et al. ${ }^{[35]}$ (b) e por Richards et al. ${ }^{[28]}$ (c). Na ref. [35] a profundidade calculada do poço é maior (133 meV) que por nós encontrada ( $85 \mathrm{meV})$. $\mathrm{Na}$ ref. [28] o valor encontrado para a profundidade do poço $(90 \mathrm{meV})$ é 

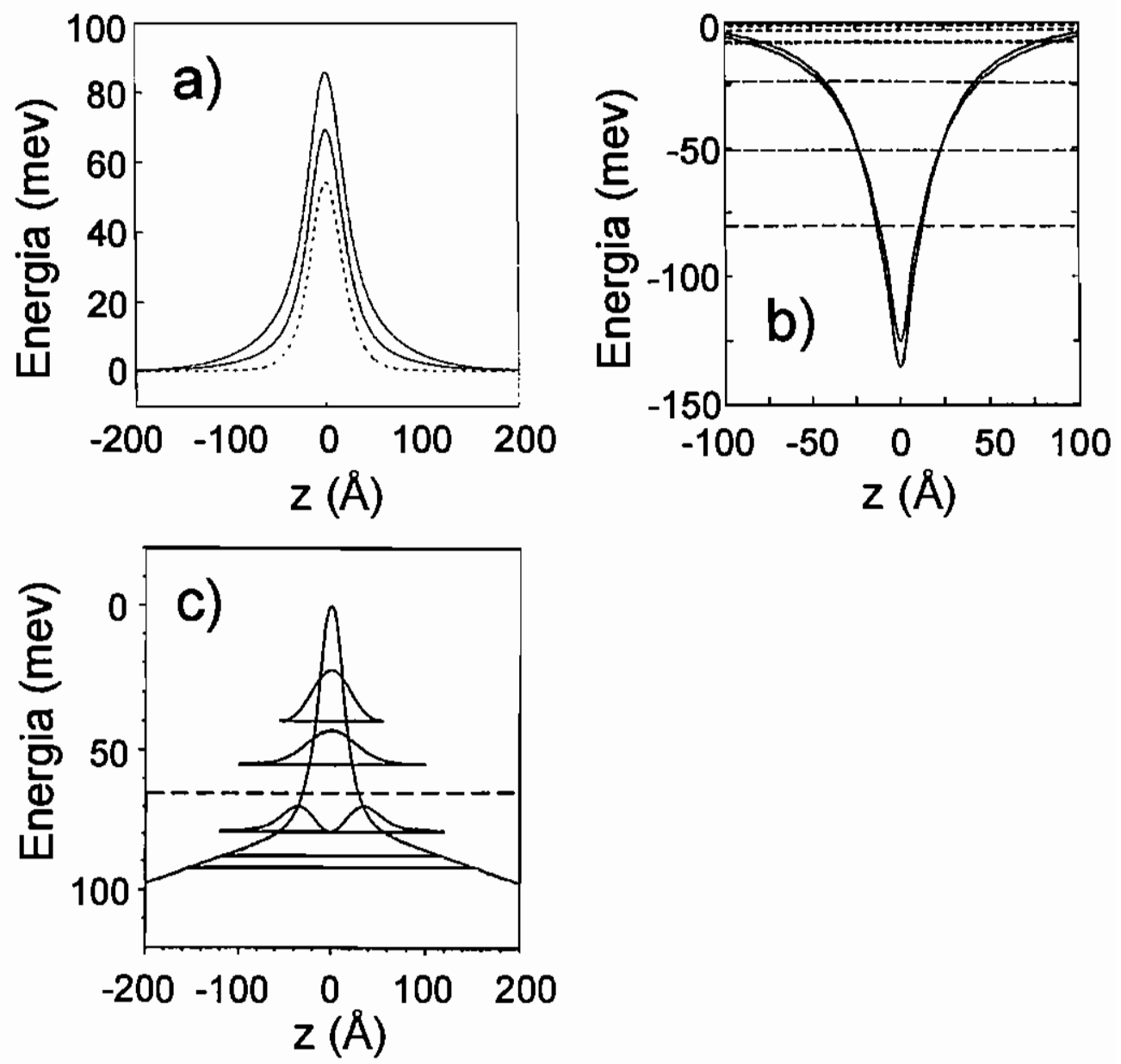

Figura 5.8: a) perfis de potencial para buracos para uma super-rede $\delta$-doping tipo p com período $d=500 \AA$, concentração de dopantes $N_{s}=8 \times 10^{12} \mathrm{~cm}^{-2}$ e $\sigma=10 \AA$. b) perfis de potencial calculados para poşo isolado com $N_{s}=8 \times 10^{12} \mathrm{~cm}^{-2}$ extraído do artigo de Reboredo et al. ${ }^{[35]}$. c) perfil de potencial, para um poço isolado de mesma concentração, extraído de Richards it et al. ${ }^{[28]}$. Não foi incluído neste último o termo de troca e correlação. 
próximo de nosso valor, porém não foi incluído neste cálculo o termo de troca e correlação do potencial. Caso este fosse incluído, o valor seria também consideravelmente superior ao por nós encontrado.

As aproximações feitas nessas duas referências parecem subestimar as densidades de estados das bandas com energias mais baixas. Em conseqüência, estados com energias mais altas, são preenchidos. Como a blindagem das cargas das impurezas pelos portadores com energias maiores são menos efetivas, o poço torna-se mais profundo. Percebese claramente que subestimam-se as densidades de cargas ao observar os níveis de Fermi: na ref. [28] ele se encontra na segunda sub-banda, $10 \mathrm{meV}$ mais baixo que em nossos cálculos, e na ref. [35] o mesmo se encontra dentro da terceira minibanda.

\subsection{Comparação entre poços $\delta$-doping tipo $\mathbf{p}$ e tipo $n$}

Um estudo comparativo de super-redes $\delta$-doping tipo $\mathrm{p}$ e tipo $\mathrm{n}$ de mesma concentração pode nos ajudar a entender melhor as diferenças entre esses dois tipos de sistema. Vamos nos concentrar nas super-redes de período $500 \AA$. A fig. 5.9 mostra à esquerda, potenciais, estados de buracos das minibandas e níveis de Fermi para poços $\delta$-doping tipo $\mathrm{p}$ de concentrações $3 \times 10^{12} \mathrm{~cm}^{-2}, 8 \times 10^{12} \mathrm{~cm}^{-2}$ e $1,5 \times 10^{13} \mathrm{~cm}^{-2}$ e para uma dispersão da camada de impurezas $\Delta z$ de $10 \AA$. À direita são mostrados potenciais, estados eletrônicos e níveis de Fermi para poços $\delta$-doping tipo n de mesma concentração e dispersão na camada de impurezas. Nas figuras, as curvas sólidas mostram os potenciais totais com a inclusão dos termos de troca e correlação, as linhas tracejadas mostram os potenciais totais sem inclusão do termo de troca e correlação e as linhas pontilhadas mostram os níveis de Fermi dos sistemas.

Ao observar-se as curvas pode-se notar que os potenciais para poços tipo n são muito mais profundos que os para poços tipo p. A blindagem do campo elétrico das impurezas pelos portadores e a localização destes últimos podem explicar o fenômeno facilmente. A distribuição dos 
5. Estrutura de Bandas
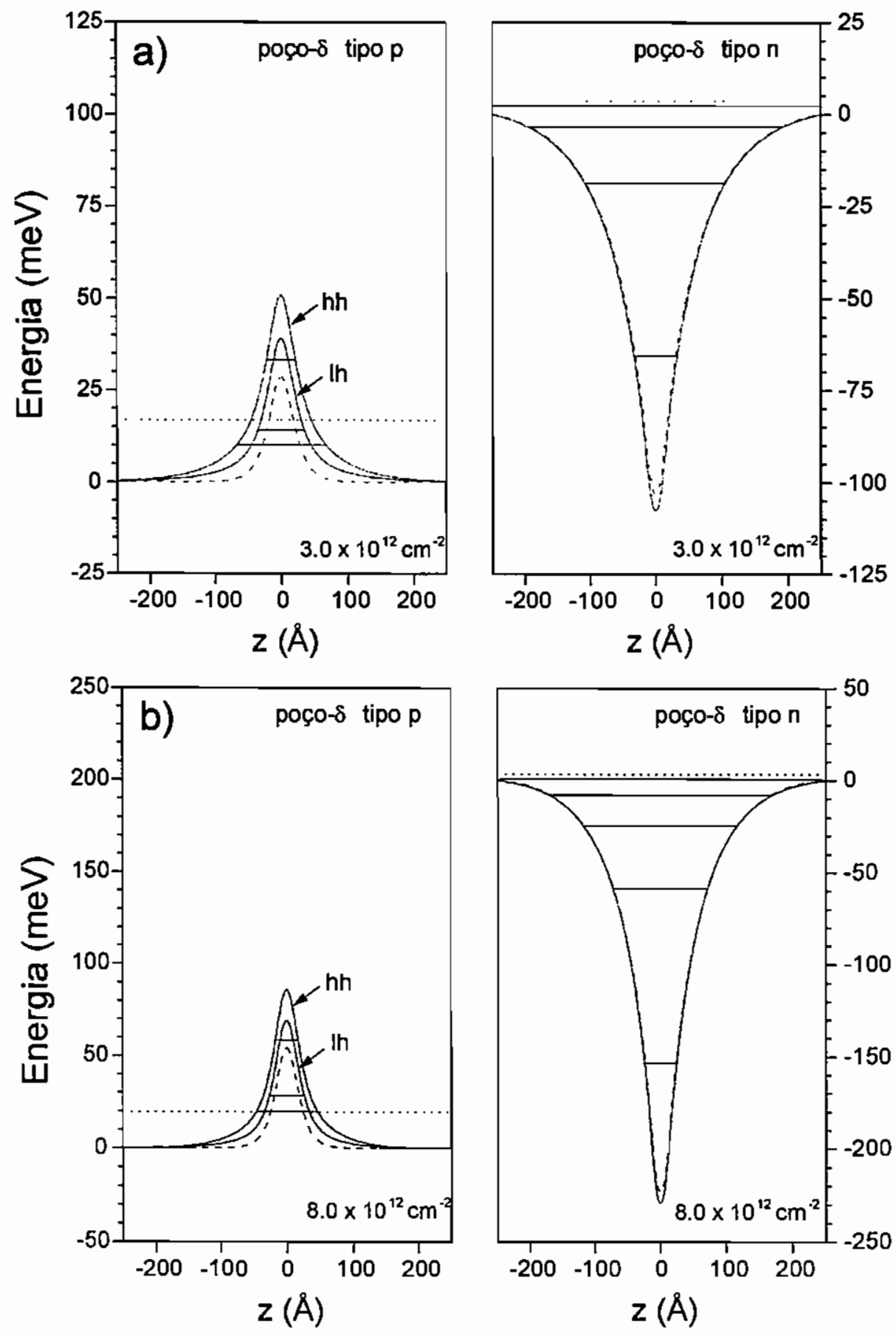
5. Estrutura de Bandas
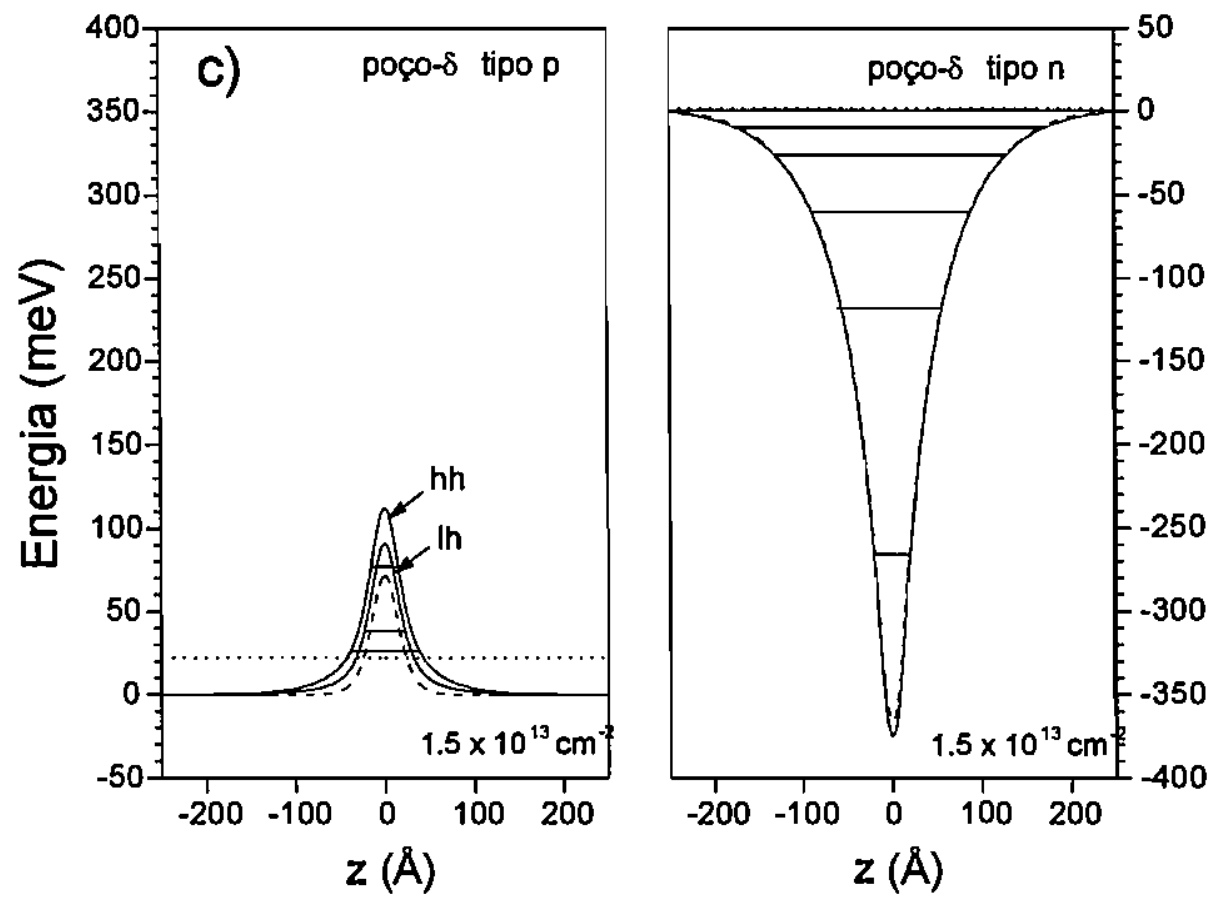

Figura 5.9: À esquerda: perfis de potencial e níveis de energia das minibandas de buracos (linhas sólidas) em uma super-rede $\delta$-doping tipo $\mathrm{p}$ com período de $500 \AA$. O potencial coulombiano (linhas tracejadas) e o nível de Fermi (linhas pontilhadas) são mostrados também. As concentrações são $3 \times 10^{12} \mathrm{~cm}^{-2}$ para o sistema a, $8 \times 10^{12} \mathrm{~cm}^{-2}$ para o sistema b e $1,5 \times 10^{13} \mathrm{~cm}^{-2}$ para o sistema c. A dispersão das amostras é de $\sigma=10 \AA$. À direita: as mesmas grandezas que do lado direito, porém para elétrons em uma super-rede $\delta$-doping tipo n de período $500 \AA$.

portadores próximo à camada de impurezas é determinada pelas suas massas efetivas. Devido à sua maior massa efetiva, os buracos pesados são muito mais localizados que os buracos leves ou os elétrons. Por esse motivo, a blindagem causada por eles é muito maior que a causada por elétrons ou buracos leves. A maior localização dos buracos pesados é responsável também por eles serem os estados fundamentais dentro do poço tipo p.

Outra diferença entre poços $\delta$-doping tipo p e tipo n, no que concerne à dependência de suas estruturas eletrônicas com a dispersão das impurezas em torno do plano de dopantes, pode ser entendida através 
de argumentos similares. Como já foi demonstrado anteriormente as alturas das barreiras e as posições dos níveis de poços $\delta$-doping tipo $\mathrm{p}$ são muito sensíveis a variações de seus perfis de dopagem. Esta característica evidencia uma diferença grande com os poços tipo $n$, os quais têm uma sensibilidade muito menor a esta grandeza ${ }^{[20]}$. A blindagem da carga do plano de dopantes pelos portadores explica esta diferença de uma maneira muito simples: como os buracos pesados dominam em poços $\delta$-doping tipo p e como estes mesmos buracos são muito mais localizados na vizinhança do plano de dopantes que os elétrons no poço $\delta$-doping tipo $n$, as variações no perfil de dopagem são sentidas com muito mais intensidade pelos buracos que pelos elétrons.

Existe ainda outra diferença fundamental entre elétrons e buracos no que diz respeito aos efeitos do potencial de troca e correlação. $\mathrm{Na}$ fig. 5.9 pode-se ver que os efeitos da troca e correlação são maiores nos buracos pesados que nos elétrons e buracos leves. Há dois motivos para tanto. Primeiro, a alta densidade de buracos pesados dá origem a um valor absoluto maior para o potencial de troca e correlação. $\mathrm{Pa}$ ra buracos pesados o seu valor é de $23 \mathrm{meV}, 32 \mathrm{meV}$ e $40 \mathrm{meV}$, para cada uma das três concentrações respectivamente, enquanto que para elétrons os valores são $5 \mathrm{meV}, 9 \mathrm{meV}$ e $12 \mathrm{meV}$. A segunda razão concerne à importância relativa do potencial de troca e correlação quando comparado ao potencial de Coulomb dos portadores e cargas fixas. O potencial coulombiano, identificado por uma linha tracejada nas fig. 5.9 , é consideravelmente menor para buracos do que para elétrons, em função da blindagem mais efetiva por parte dos buracos pesados. $\mathrm{Pa}$ ra ilustrar pode-se tirar da fig. 5.9 os valores relativos do potencial de troca e correlação. Este é, para buracos pesados, $44 \%, 30 \%$ e $30 \%$ do potencial coulombiano, respectivamente. Para elétrons os valores são $6 \%, 4 \%$ e $3 \%$. Por último, as diferentes profundidades dos poços $\delta$-doping tipo p e tipo $\mathrm{n}$ podem resultar em diferentes intensidades dos picos de luminescência referentes às regiões $\delta$-doping. A dificuldade de um buraco tunelar por uma barreira de potencial muito mais profunda que aquela pela qual o elétron teria de tunelar é um dos fatores que explicam a presença de luminescência em poços $\delta$-doping tipo $\mathrm{p}$ isolados, enquanto que para poços tipo $n$ ela é inexistente. 


\section{Capítulo 6}

\section{Fotoluminescência de Estruturas $\delta$-doping tipo $\mathbf{p}$ em GaAs}

Nesta seção apresentaremos espectros de fotoluminescência calculados com o método descrito no capítulo 4. Os parâmetros para o modelo de Kane usados foram: $m_{e}=0,0665 m_{0}, \overline{\gamma_{1}}=0,3983, \overline{\gamma_{2}}=-1,2714$, $\overline{\gamma_{3}}=-0,4136$ e $E_{p}=29,12 \mathrm{eV}$. O valor usado para o 'gap' do GaAs a $\mathrm{T}=0 \mathrm{~K}$ foi $1519 \mathrm{meV}$.

Os dados serão apresentados da seguinte maneira: primeiro consideraremos uma super-rede com largura de $500 \AA$, concentração de dopantes $N_{A}=8 \times 10^{12} \mathrm{~cm}^{-2}$, com um perfil de dopagem Gaussiano de $\sigma=10 \AA$. Para esta super-rede apresentaremos os espectros de luminescência em diversas temperaturas. Mostraremos ainda para este sistema o potencial autoconsistente, a estrutura de minibandas e sub-bandas de energia, as funções de onda de elétrons e buracos e a integral de superposição entre elétrons e buracos. Em seguida, apresentaremos espectros calculados para sistemas cujos dados experimentais estão disponíveis na literatura. Por último mostraremos e discutiremos a dependência em temperatura da intensidade do espectro de luminescência a qual é dramática. 


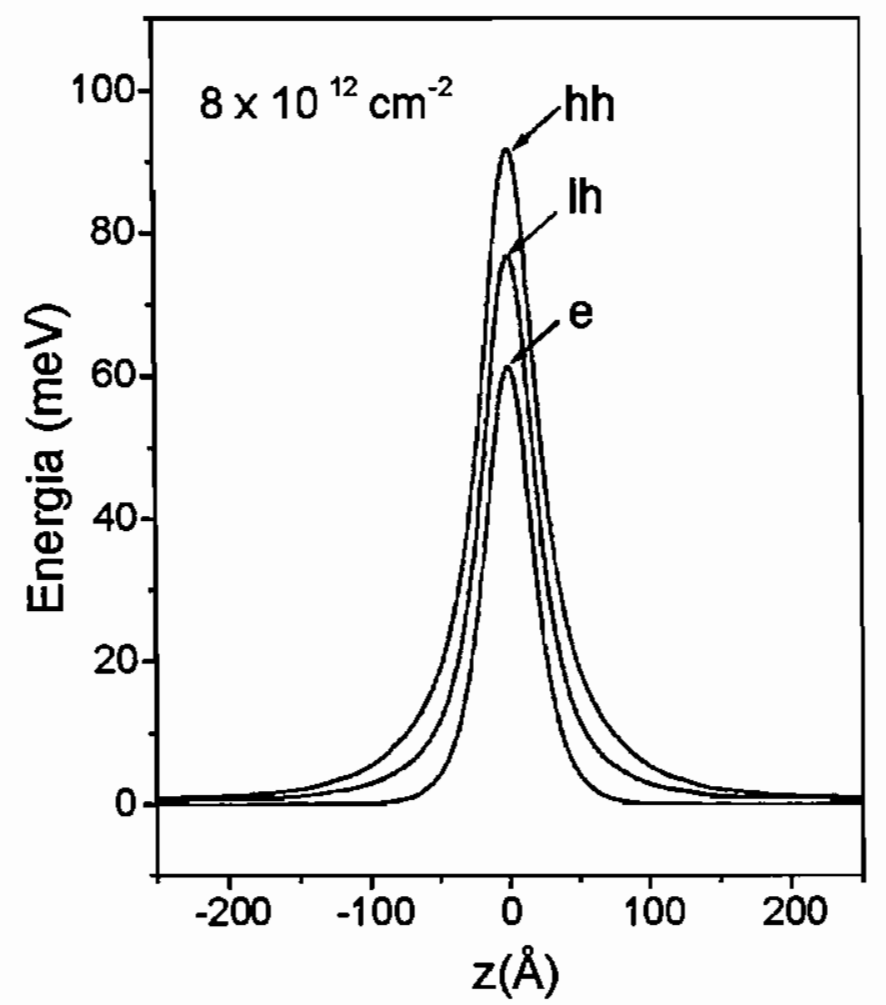

Figura 6.1: Potencial autoconsistente para elétrons, buracos pesados e buracos leves. Super-rede com $N_{A}=8 \times 10^{12} \mathrm{~cm}^{-2}, \Delta z=23.5 \AA$ e d $=500 \AA$.

\subsection{Super-rede típica}

\subsubsection{Potencial autoconsistente e estrutura de bandas}

O potencial autoconsistente mostrado na fig. 6.1 foi calculado segundo a aproximação de Kane. Como podemos notar ao compararmos o potencial obtido com essa aproximação e o potencial obtido com aproximação de Luttinger-Kohn (ver fig. 5.9), não há diferença significativa entre os potenciais de buracos calculados por uma aproximação ou pela outra. Para o estudo da luminescência de uma amostra é, no entan- 


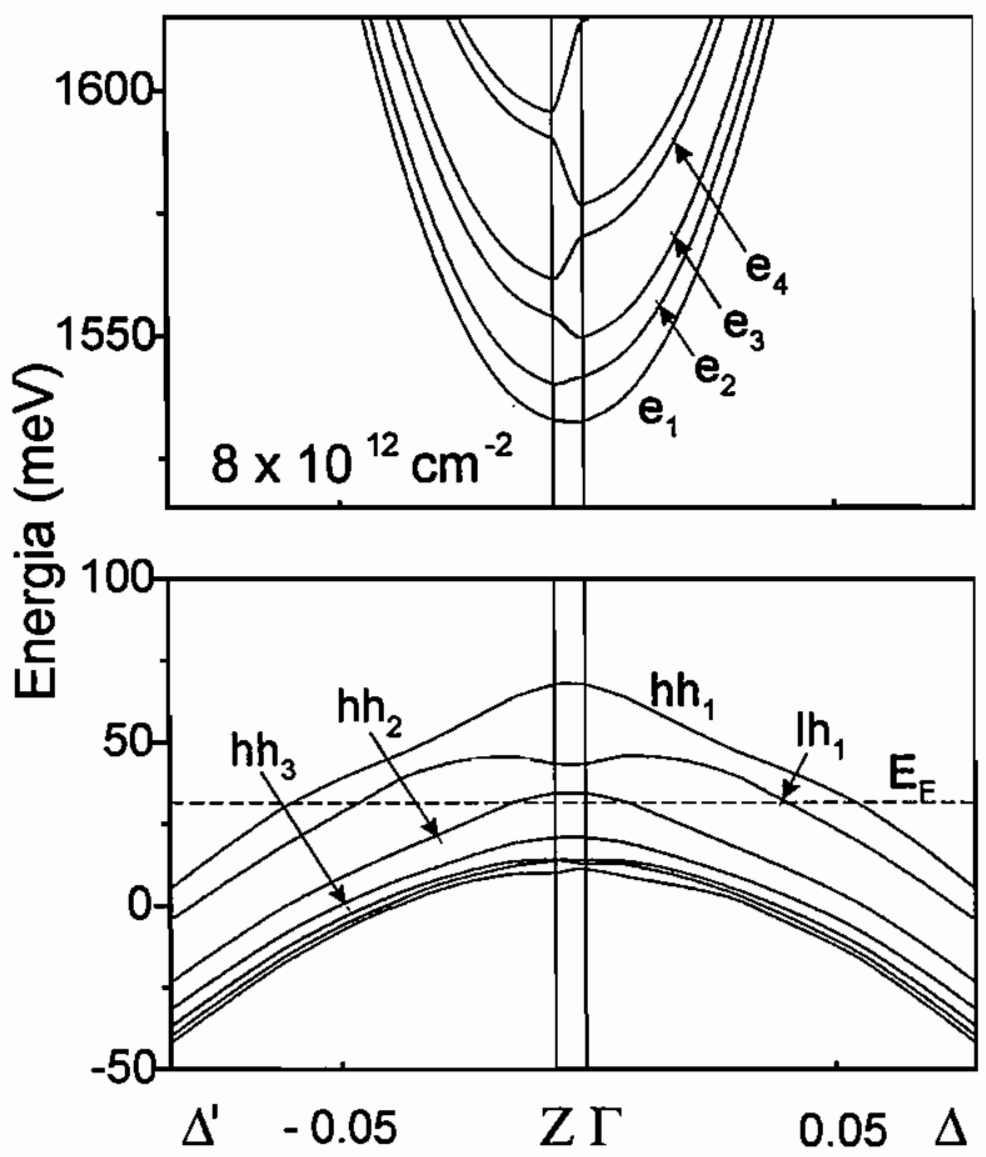

Figura 6.2: Curvas de dispersão para sub-bandas e minibandas para elétrons (em cima) e buracos (embaixo). Mesmo sistema da fig. 6.1.

to, necessário a presença de elétrons na banda de condução do sistema e por conseqüência devemos determinar o potencial que eles sentem. Como já foi discutido anteriormente, o potencial que partículas de um determinado tipo sentem é diferente do potencial percebido por uma partícula de um tipo diferente, porque o termo de troca e correlação do potencial depende da concentração específica de cada tipo de portador. Por serem os elétrons os portadores minoritários, que devido à recombinação com os buracos têm uma vida média muito pequena, sua concentração é desprezível, o que torna o termo de troca e correlação de seu potencial praticamente nulo. Portanto, o potencial total sentido 
pelos elétrons é o potencial coulombiano produzido pelas impurezas e pelos buracos da amostra.

As estruturas das minibandas e sub-bandas da super-rede são mostradas na fig. 6.2 para elétrons e buracos. A dispersão das sub-bandas na direção paralela à camada de dopantes mostra o espalhamento entre as bandas típico de sistemas tipo p em GaAs. A dispersão das minibandas é pequena devido à largura da barreira. Duas sub-bandas de buracos pesados e uma de buracos leves estão parcialmente ocupadas. A comparação das estruturas de buracos no modelo de Kane com as estruturas no modelo de Luttinger-Kohn (ver fig. 5.6) mostra uma vez mais que a diferença entre os dois modelos não é significativa no que concerne à estrutura da banda de valência.

Até aqui consideramos super-redes com período $d \geq 500 \AA$ como poços isolados. Para a determinação do potencial autoconsistente em estruturas $\delta$-doping tipo $\mathrm{p}$, essa aproximação é válida. Para o cálculo dos espectros de fotoluminescência porém, esta aproximação já não é válida. Buracos em poços adjacentes em super-redes com período $d \geq 500 \AA$ não sentem buracos de poços vizinhos, mas no que concerne aos elétrons confinados entre as duas barreiras, essa afirmação não é verdadeira. Ao observarmos a estrutura da banda de condução na fig. 6.2, vemos que a separação entre as minibandas é de $20 \mathrm{meV}$ ou mais. Para que pudéssemos, em nosso modelo, fazer a aproximação de um contínuo de estados, simulando o limite do período tendendo a infinito, deveríamos ter 'minigaps' com valor menor que $10 \mathrm{meV}$, o que só obtemos com super-redes de $2000 \AA$ ou mais. Portanto consideraremos para efeito da luminescência poços isolados somente super-redes com períodos maiores que $2000 \AA$.

\subsubsection{Funções envelope}

Dada a existência de um potencial periódico na direção perpendicular à camada $\delta$, as funções envelope de buracos e elétrons nesta direção 


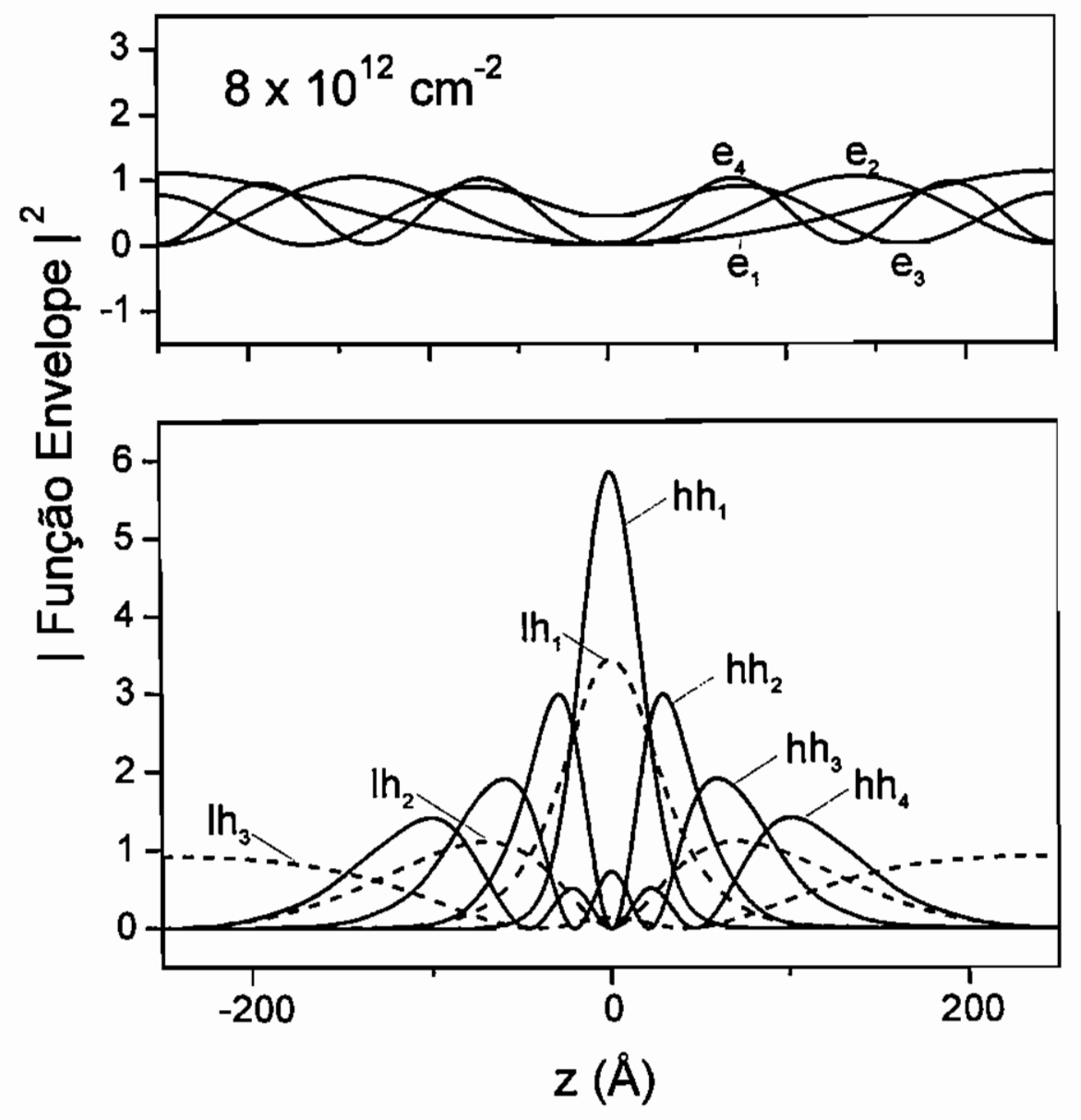

Figura 6.3: Funções de onda ao quadrado para elétrons (em cima) e buracos (embaixo). Mesmo sistema da fig. 6.1.

são funções de Bloch $\left(z \mid n_{x} q_{z}\right)$, com $x=e, l h, h h$, enquanto que nas direções paralelas à camada podem ser expandidas em ondas planas. Os módulos ao quadrado das funçōes de Bloch no centro da primeira zona de Brillouin $(\Gamma)$ são mostrados na fig. 6.3. Mais uma vez a diferença do comportamento de elétrons e buracos em relação à camada de dopantes é fundamental. Os buracos estão confinados na região dos dopantes e o módulo de sua função de onda decai exponencialmente fora dela, enquanto os elétrons estão praticamente livres e o módulo de sua função decai dentro da região dos dopantes. 


\subsubsection{Forças de oscilador}

Conhecidas as autofunções e estruturas de bandas de elétrons e buracos, podemos passar ao cálculo do espectro de luminescência $I(\omega)$ da nossa super-rede típica através da expressão (4.4). Nesta expressão as autofunções são levadas em conta através da força de oscilador $f_{n_{e} n_{q h}}$ descrita pela equação (4.5). A fig. 6.4 mostra a força de oscilador para transições para os dois estados mais profundos de buracos leves e pesados, no centro da $1 \underline{a}$ Zona de Brillouin. Essa curva foi obtida plotando $f_{n_{e} l_{q h}}$ em função da energia $E_{n_{e}}(0)$ das várias minibandas $n_{e}$ em $\Gamma$.

Nas transições para o estado fundamental de buracos pesados, $f_{n_{e} l_{h h}}(0)$ cresce rapidamente entre a primeira e a segunda minibandas, alcançando seu máximo na última. A partir deste ponto, $f_{n_{e} l_{h h}(0)}$ decresce continuamente. Isto reflete a variação da superposição espacial entre as funções envelope de elétrons e buracos: as funções de elétrons com baixa energia decaem rapidamente quando penetram as barreiras. Em conseqüência, a superposição destas funções com as de buracos é pequena. À medida que a energia aumenta, a penetração da função eletrônica na barreira aumenta e a superposição aumenta. Um aumento ainda maior da energia da função eletrônica, faz com que ela oscile cada vez mais rapidamente e a integral de superposição volte a cair, pois a média do valor da função envelope na região em que ela se superpõe às funções dos buracos tende a zero.

Nas transições para o estado fundamental de buracos leves, a força de oscilador $f_{n_{e} l_{l h}}(0)$ mostra o mesmo comportamento que no caso de buracos pesados. Para a primeira minibanda de elétrons, $f_{n_{e} 1_{l h}}(0)$ tem praticamente o mesmo valor que $f_{n_{e} 1_{h h}}(0)$. Se levássemos em conta apenas os quadrados dos elementos da matriz de momentos entre as bandas de valência e de condução, o valor da força de oscilador entre os elétrons e os buracos pesados deveria ser 6 vezes maior que a força de oscilador entre elétrons e buracos leves. No caso que estudamos, a força de oscilador para buracos leves é aumentada porque estes penetram mais na barreira de potencial que os buracos pesados. Nas transiçōes da segunda minibanda eletrônica, a força de oscilador dos buracos leves é metade da dos buracos pesados. Com o aumento do

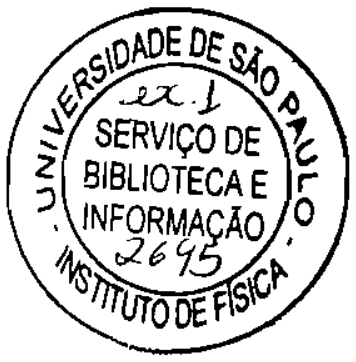




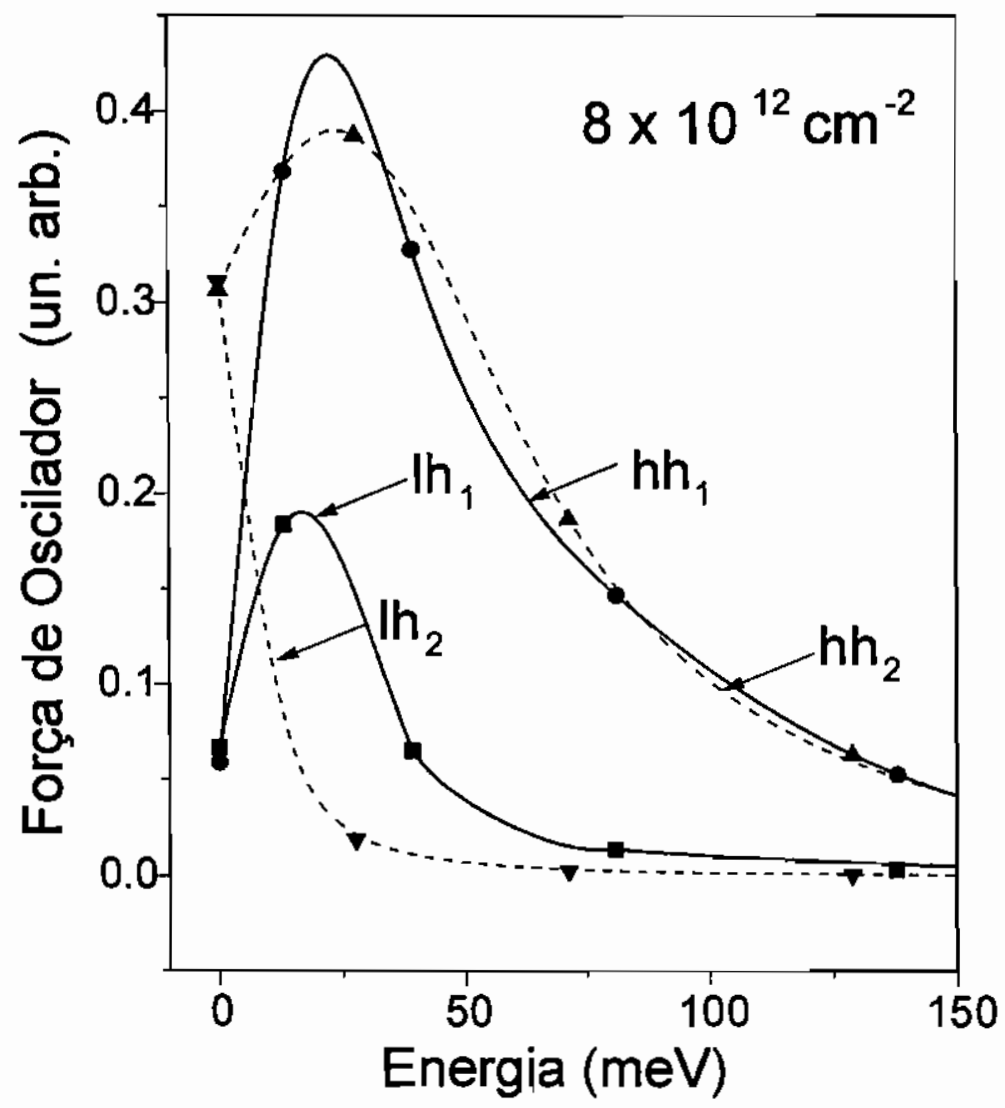

Figura 6.4: Forças de oscilador para transições ópticas de estados eletrônicos para os estados fundamentais de buracos leves e pesados no centro da primeira Zona de Brillouin, mostrada em função da energia do elétron. Mesmo sistema da fig. 6.1.

número da minibanda eletrônica, a força de oscilador para buracos leves perde rapidamente a intensidade quando comparada à dos buracos pesados. Essa queda do valor da função de superposição é causada como no caso dos buracos pesados, pela média do valor da função envelope na região de superposição e é acentuada pelo fato do elemento da matriz de momento ser menor.

As transições para os primeiros estados excitados são qualitativamente iguais às transições para os estados fundamentais, tanto para buracos leves quanto para buracos pesados. Os valores absolutos refletem a 
maior penetração dos estados na barreira.

\subsubsection{Espectros de fotoluminescência}

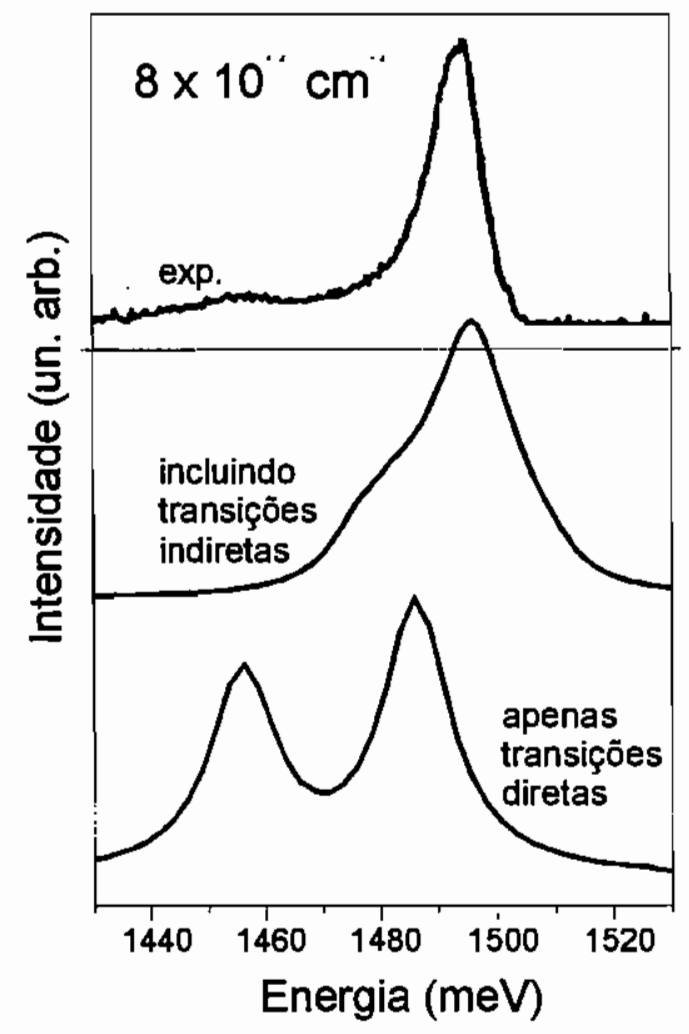

Figura 6.5: Espectros calculados de fotoluminescência para o mesmo sistema da fig. 6.1. São mostrados os espectros considerando apenas transições diretas ou considerando transições diretas e indiretas. $\mathrm{O}$ alargamento causado pelo tempo de decaimento do portador minoritário é de $5 \mathrm{meV}$. Um espectro experimental, extraído de Wagner et al. ${ }^{[26]}$, é mostrado para comparação.

$\mathrm{Na}$ fig. 6.5 são mostrados espectros de fotoluminescência calculados para nossa super-rede típica incluindo apenas transições diretas ou transições diretas e indiretas. A temperatura utilizada no cálculo foi $\mathrm{T}=6 \mathrm{~K}$, e o valor de $5 \mathrm{meV}$ foi utilizado para o alargamento devido ao tempo de vida finito do portador minoritário. O espectro para 
transições diretas mostra dois picos abaixo do valor do 'gap'. Estes picos podem ser claramente interpretados como transições entre estados eletrônicos e estados de buracos próximos ao ponto $\Gamma$, sendo o pico de mais baixa energia associado a transições para o estado fundamental de buracos pesados $h h_{1}$ e o de mais alta energia a transições para o estado fundamental de buracos leves $l h_{1}$, as duas únicas bandas parcialmente preenchidas em nosso caso (ver fig. 6.2). O pico devido aos buracos leves é mais intenso que o devido aos buracos pesados. Um exame das forças de oscilador mostradas na fig. 6.4 mostra a razão desse comportamento não usual: a energia eletrônica média $k T$, medida do fundo da banda mais baixa é cerca de $0.5 \mathrm{meV}$. Para energias tão pequenas, as forças de oscilador para buracos leves e pesados são praticamente iguais. A diferença de energia entre os dois picos é de $30 \mathrm{meV}, 5 \mathrm{meV}$ maior que a separação entre as bandas mais baixas de buracos leves e pesados.

O espectro de fotoluminescência incluindo também transições indiretas mostra-se completamente diferente. Um pico muito intenso aparece à energia do 'gap' e ligeiramente acima dele, e abaixo do 'gap' um segundo pico se superpõe a este primeiro. As transições de estados eletrônicos para os estados fundamentais de buracos leves e pesados no centro da Zona de Brillouin $(\Gamma)$ misturam-se e são responsáveis pelo pico menor. O pico mais intenso próximo ao valor do 'gap' é causado por recombinações para estados das minibandas $h h_{1}$ e $l h_{1}$ fora do ponto $\Gamma$.

Por último a fig. 6.5 mostra também um espectro experimental ${ }^{[26]}$ de uma amostra comparável à do sistema aqui estudado, um poço $\delta$-doping tipo $\mathrm{p}$ entre duas barreiras de AlGaAs, formando um poço quadrado de $600 \AA$. Nos espectros teóricos, as barreiras de AlGaAs são simuladas pela interface entre duas supercélulas subseqüentes. Ao compararmos os espectros desta figura, nota-se que o espectro teórico que leva em conta transições indiretas ajusta-se muito melhor ao espectro obtido experimentalmente que o que leva em conta apenas transições diretas. Isto continua sendo verdade caso se aumente o período da super-rede ou se aumente o parâmetro de alargamento da banda referente ao tempo de vida finito do elétron. Pode-se então concluir que o processo dominante de recombinação radiativa nessa amostra são transições indiretas. 
Um ajuste perfeito do espectro calculado com o espectro experimental não deve ser esperado, visto que a medida da dispersão das impurezas tem uma incerteza considerável e que o potencial calculado pode diferir do experimental por causa do alinhamento ('pinning') do nível de Fermi na superfície da amostra. O potencial e outras grandezas associadas podem ainda ser modificadas pela potência de excitação do laser. Isto deve resultar em variaçōes do espectro de fotoluminescência em função da potência, como é observado experimentalmente. O espectro experimental mostrado na fig. 6.5 é o medido com a menor potência de excitação na amostra $(3 \mathrm{~mW})$, o qual deve ser o mais próximo de nossos cálculos.

\subsection{Espectros de fotoluminescência para diversas super-redes $\delta$-doping tipo p}

Os sistemas apresentados na fig. 6.6 são comparáveis às amostras da fig.4 da ref. [28]. Como a amostra experimental da fig. 6.5, essas amostras se referem a poços $\delta$-doping tipo p em GaAs entre duas barreiras de AlGaAs separadas entre si por $600 \AA$. As concentraçōes e a dispersão das impurezas em torno do plano de dopantes são: $N_{A}=3 \times 10^{12} \mathrm{~cm}^{-2}$ com $\Delta z=65 \AA$ (amostra 2), $N_{A}=8 \times 10^{12} \mathrm{~cm}^{-2}$ com $\Delta z=72 \AA$ (amostra 3) e $N_{A}=3 \times 10^{13} \mathrm{~cm}^{-2}$ com $\Delta z=125 \AA$ (amostra 4). A barreira, como no sistema anterior, é simulada pelas condições de contorno. Os espectros mostrados levam em conta transições indiretas. Olhando de uma maneira global, a concordância entre os espectros experimentais e o calculados é muito boa. Para a amostra 2, temos um único pico intenso, para a amostra 3 temos um pico mais largo com um ombro na parte referente às energias mais altas, e na amostra 4 um pico intenso com dois picos largos em energias mais baixas. $O$ ganho de intensidade no nível de Fermi ('Fermi Level Enhancement') indicado nos espectros experimentais não é reproduzido em nossos cálculos porque não foram levados em conta efeitos de muitos corpos tipo éxciton relacionados ao nível de Fermi. 


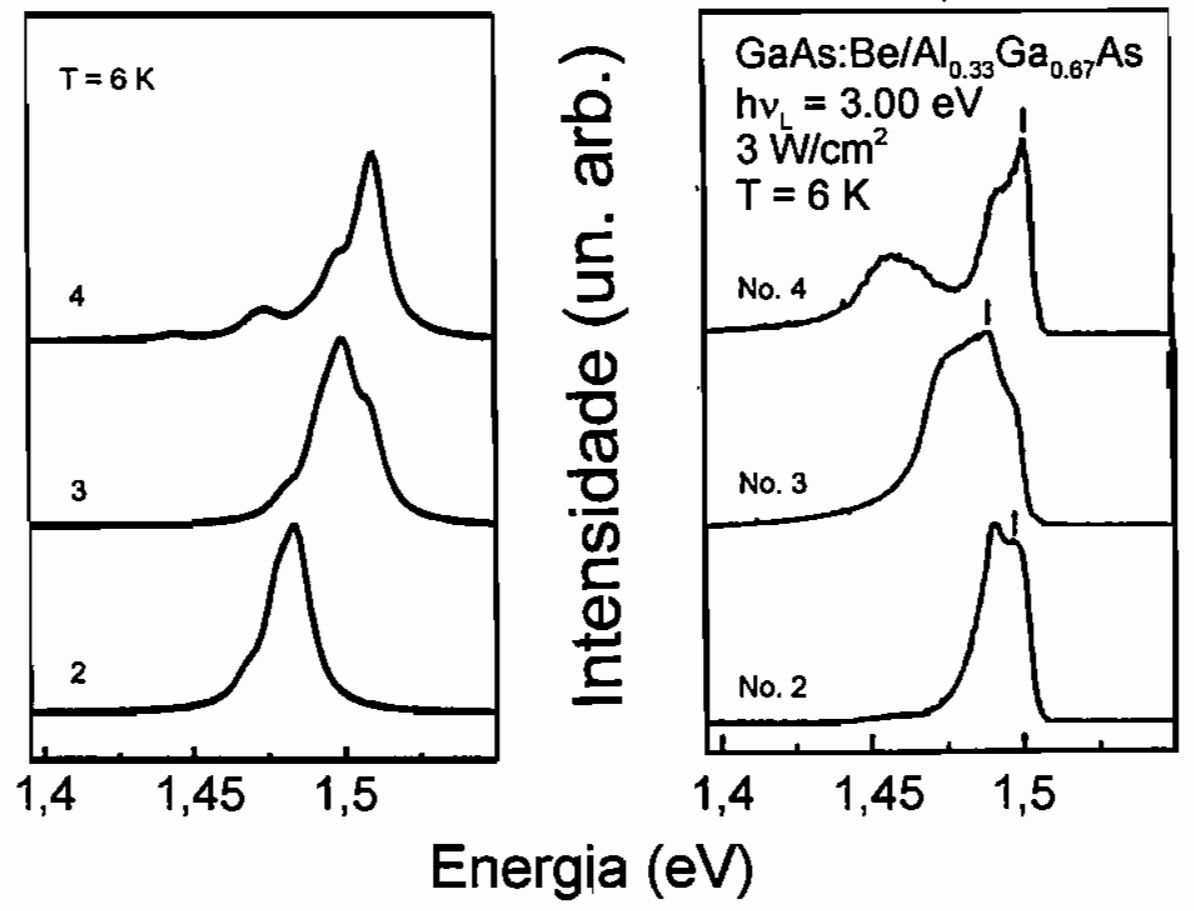

Figura 6.6: $\grave{A}$ esquerda: espectros calculados para super-redes $\delta$-doping tipo p com período de $600 \AA$ e várias concentrações $\left(N_{A}=3 \times 10^{12} \mathrm{~cm}^{-2} \mathrm{com}\right.$ $\Delta z=65 \AA$ para a, $N_{A}=8 \times 10^{12} \mathrm{~cm}^{-2} \operatorname{com} \Delta z=72 \AA$ para b e $N_{A}=$ $3 \times 10^{13} \mathrm{~cm}^{-2}$ com $\Delta z=125 \AA$ para c). $\AA$ direita: espectros experimentais para poços $\delta$-doping inseridos entre barreiras de AIGaAs separadas entre si por $600 \AA^{[28]}$. As amostras experimentais e os sistemas calculados têm rigorosamente as mesmas concentrações e dispersões.

Apesar das semelhanças na forma, os picos dos espectros calculados são desviados para energias mais altas com o aumento da concentração enquanto que os picos dos espectros experimentais permanecem praticamente à mesma energia. Atribuímos isso à redução do valor do 'gap' de energia com o aumento da concentração ('gap shrinkage') que não é levada em conta em nosso cálculo. À luz dessa interpretação, estimamos uma redução do valor do 'gap' de $50 \mathrm{meV}$ entre as concentrações $N_{A}=3 \times 10^{12} \mathrm{~cm}^{-2}$ e $N_{A}=3 \times 10^{13} \mathrm{~cm}^{-2}$. 
6. Fotoluminescência de Estruturas $\delta$-doping tipo p em $\mathrm{GaAs} 106$
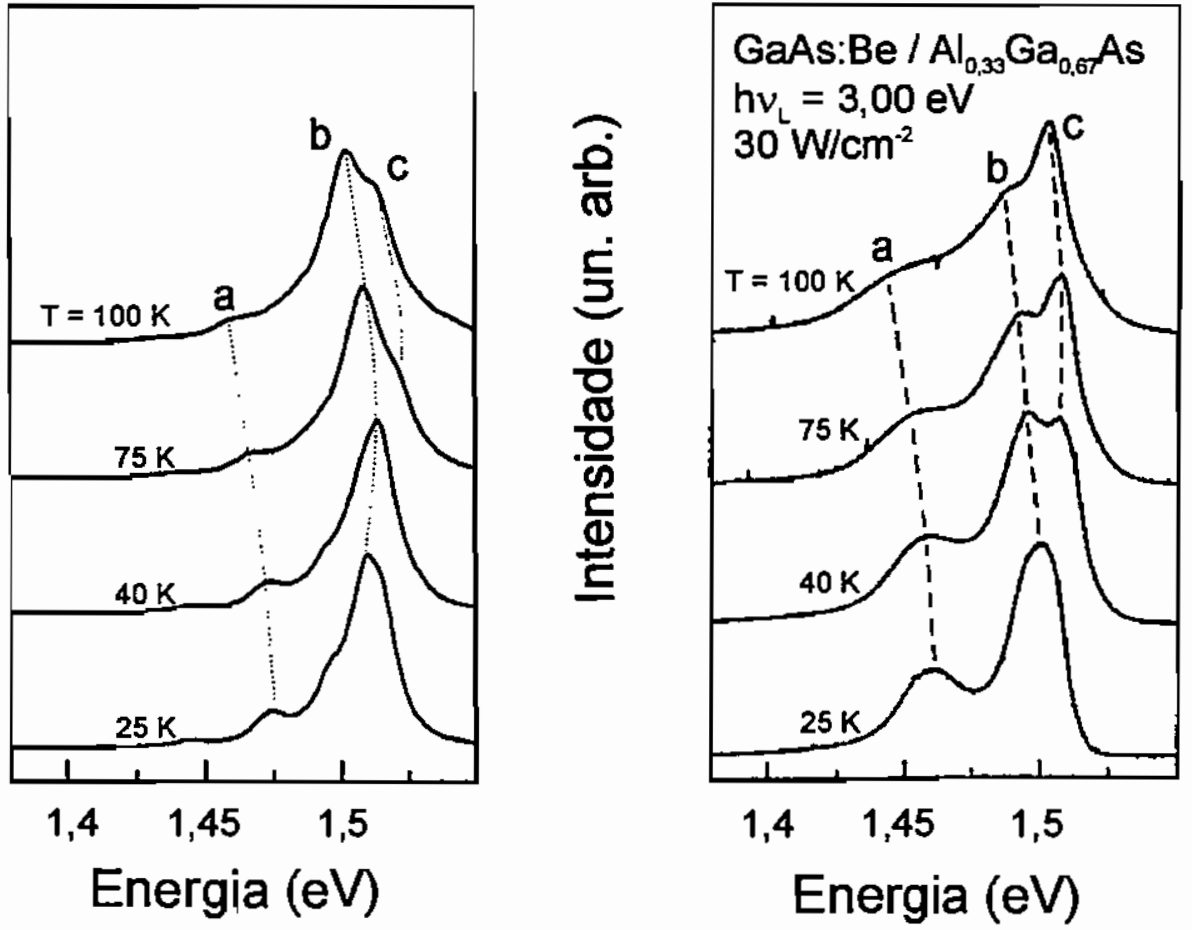

Figura 6.7: $\grave{A}$ esquerda: espectros calculados para super-redes $\delta$-doping tipo p com período de $600 \AA, N_{A}=3 \times 10^{13} \mathrm{~cm}^{-2}$ com $\Delta z=125 \AA$ e diferentes temperaturas. De baixo para cima: $\mathrm{T}=25 \mathrm{~K}, \mathrm{~T}=40 \mathrm{~K}, \mathrm{~T}=75 \mathrm{~K}$, $\mathrm{T}=100 \mathrm{~K} . \grave{A}$ direita: espectros experimentais tomados para as mesmas temperaturas, de um sistema com um poço $\delta$-doping de mesma concentração e dispersão, inserido entre duas barreiras de AlGaAs separadas entre si por $600 \AA^{[28]}$.

\subsection{Dependência do espectro com a temperatura}

Uma questão interessante que se coloca é a da variação do espectro de fotoluminescência com a temperatura. A fig. 6.7 mostra, à esquerda, os espectros calculados para uma super-rede com $N_{A}=3 \times 10^{13} \mathrm{~cm}^{-2}$ e $\Delta z=125 \AA$, a quatro diferentes temperaturas, assumindo um alargamento por tempo de decaimento de $5 \mathrm{meV}$. O pico de baixas energias (a) move-se monotonicamente para energias mais baixas com 
o aumento da temperatura. O pico principal (b) move-se para energias mais altas entre $25 \mathrm{~K}$ e $40 \mathrm{~K}$ e de volta a energias mais baixas entre $40 \mathrm{~K}$ e $100 \mathrm{~K}$. Um terceiro pico (c) aparece superposto ao pico principal a $75 \mathrm{~K}$ e move-se na direção de baixas energias com o aumento da temperatura. O movimento dos três picos em direção a energias menores é devido à redução do 'gap' de energia em função da temperatura. Um comportamento experimental semelhante foi observado em amostras com os mesmos parâmetros dos sistemas calculados(fig. 6.7, à direita). A única variação nos parâmetros relacionada ao aumento de temperatura foi a redução do valor do 'gap'.

$\mathrm{Na}$ fig. 6.7 os espectros são normalizados em relação ao pico de maior intensidade, não se levando em conta a intensidade real. Os espectros mostrados à esquerda da fig. 6.8 mostram a dependência do espectro com a temperatura para uma super-rede de período $\mathrm{d}=2000 \AA$, $N_{A}=8 \times 10^{12} \mathrm{~cm}^{-2}$ e dispersão $\Delta z=23.55 \AA$. O período grande permite considerarmos o poço isolado e os elétrons livres. Para essa amostra, as bandas $h h_{1}, l h_{1}$ e $h h_{2}$ estão parcialmente ocupadas. Cada um desses espectros, exibe uma estrutura definida, causada pelas transições da banda de condução para os três estados parcialmente ocupados. Com o aumento de temperatura os espectros tornam-se mais largos, crescendo em direção às energias mais altas. A razão desse alargamento é a seguinte: com o aumento da temperatura, os elétrons da banda de condução são excitados e em decorrência disto, estados eletrônicos em energias mais altas passam a ser parcialmente ocupados. Como as integrais de superposição de transições a partir de estados com energia maior têm valores mais altos, as transições de energia mais alta ganham intensidade e alargam a emissão.

A observação experimental mostra, no entanto, um comportamento oposto ao esperado por nosso modelo: a intensidade da emissão dos buracos confinados diminui com o aumento da temperatura. Isto pode ser observado na da fig. $6.9 \mathrm{~b}$, que apresenta medidas da luminescência de uma mesma amostra $\left(N_{A}=3,9 \times 10^{12} \mathrm{~cm}^{-2}\right.$ e $\Delta z$ não mencionado) sob uma energia de excitação de $100 \mathrm{~W} \mathrm{~cm}^{-2}$ e a temperaturas variando entre $\mathrm{T}=5 \mathrm{~K}$ e $\mathrm{T}=60 \mathrm{~K}$ (A escala de energias encontra-se invertida e a de intensidades é logarítmica). Os picos assinalados com a 

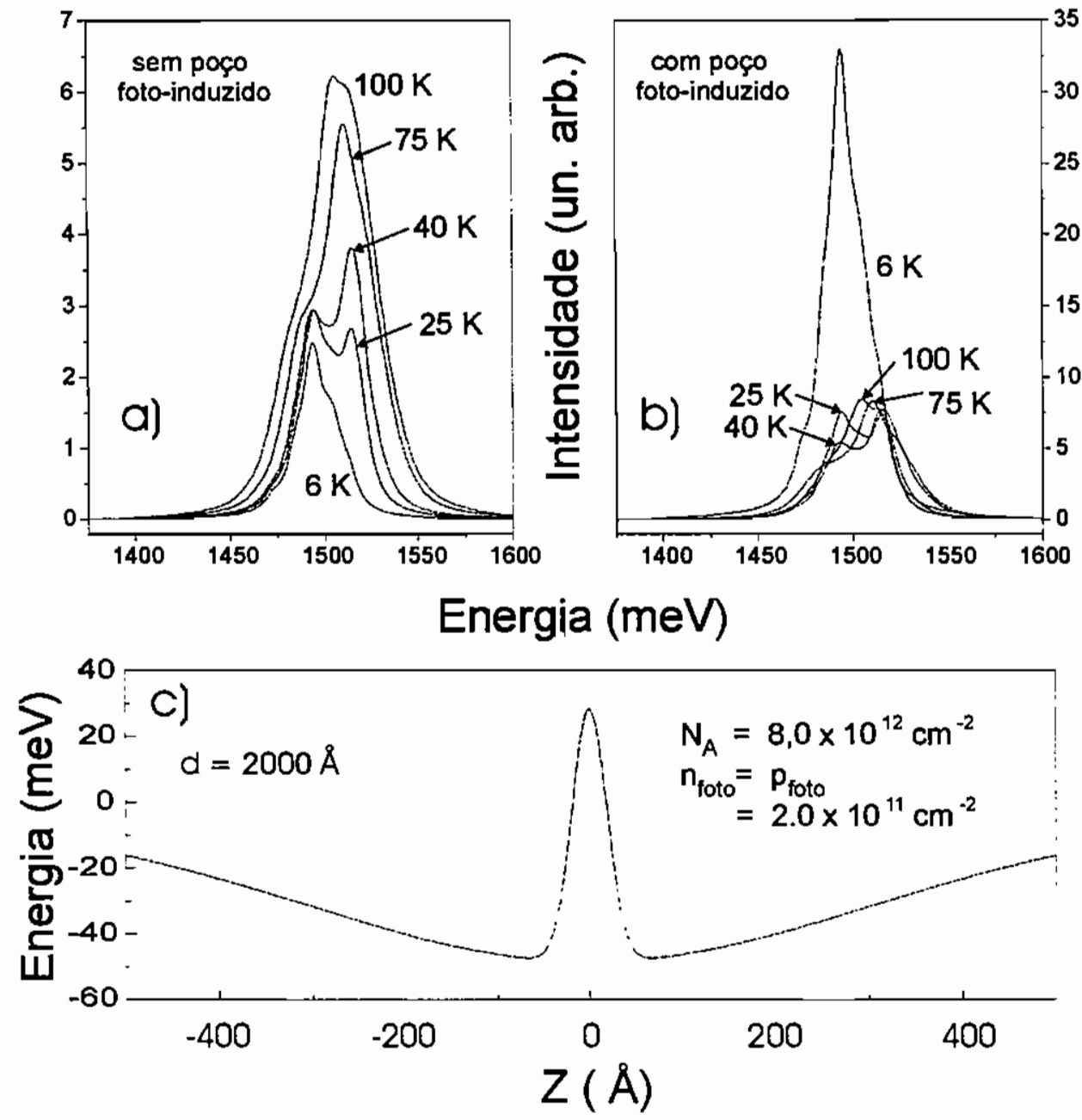

Figura 6.8: Espectros de fotoluminescência teóricos calculados para poços $\delta$-doping tipo $\mathrm{p}$ isolados. a) sem o potencial dos portadores fotocriados e b) com o potencial dos portadores fotocriados. Na parte inferior da figura (c) é apresentado o potencial autoconsistente do sistema com a inclusão de portadores fotocriados. 
letra A são devidos a recombinações excitônicas relacionadas ao GaAs volumétrico (Como este modo de recombinação não está presente no nosso modelo, não se pode esperar que esse pico seja descrito por nosso programa de cálculo). Em uma energia menor que a do pico A, vê-se uma estrutura causada pelas transições para os estados ocupados (C). A intensidade do pico A se reduz em duas ordens de grandeza com o aumento de temperatura entre $2 \mathrm{~K}$ e $60 \mathrm{~K}$. Isto é devido principalmente a processos de recombinação não-radiativa, que ocorrem mais freqüentemente a temperaturas mais altas. A intensidade do pico devido às transições para estados confinados no poço também deveria diminuir duas ordens de grandeza se essa fosse a única razão de sua redução. $\mathrm{Na}$ verdade sua redução é de quatro ordens de grandeza. Levando-se em conta que a redução global da intensidade do espectro é de duas ordens de grandeza, temos uma redução adicional de duas ordens de grandeza para o pico relativo aos estados confinados dentro do poço. Essa redução dramática foi também observada em poços $\delta$-doping tipo $\mathrm{p}$ em $\mathrm{Si}^{[53]}$. Para explicar qualitativamente este comportamento, os autores da ref. [53] assumem uma concentração fotoinduzida de portadores.

A luz incidente dissocia pares de portadores, gerando uma carga extra de buracos (portadores majoritários) e elétrons (portadores minoritários). Os buracos são atraídos para o poço, blindando o potencial repulsivo para os elétrons. Com a blindagem do potencial repulsivo e um acréscimo de carga positiva dentro do poço forma-se um poço de potencial atrativo para elétrons, com menor profundidade na borda do poço original. Os elétrons que a princípio seriam repelidos, passam a ser confinados nesse segundo poço, aumentando, por causa do confinamento, a integral de superposição de suas funções com os buracos e em conseqüência, as forças de oscilador das transições. Com o aumento da temperatura, os elétrons confinados por este poço são excitados até estados delocalizados, que têm força de oscilador menor, e a intensidade do pico diminui. Estudamos o modelo sugerido utilizando um número de buracos maior que o número de aceitadores, simulando assim o aumento da concentração de buracos devido aos portadores fotogerados. A neutralidade de cargas, assumida em nossos cálculos, cria os elétrons fotogerados automaticamente. Na fig. $6.8 \mathrm{c}$ nós mostramos o perfil de potencial para o mesmo sistema da fig. 6.8 a com um fundo de buracos 

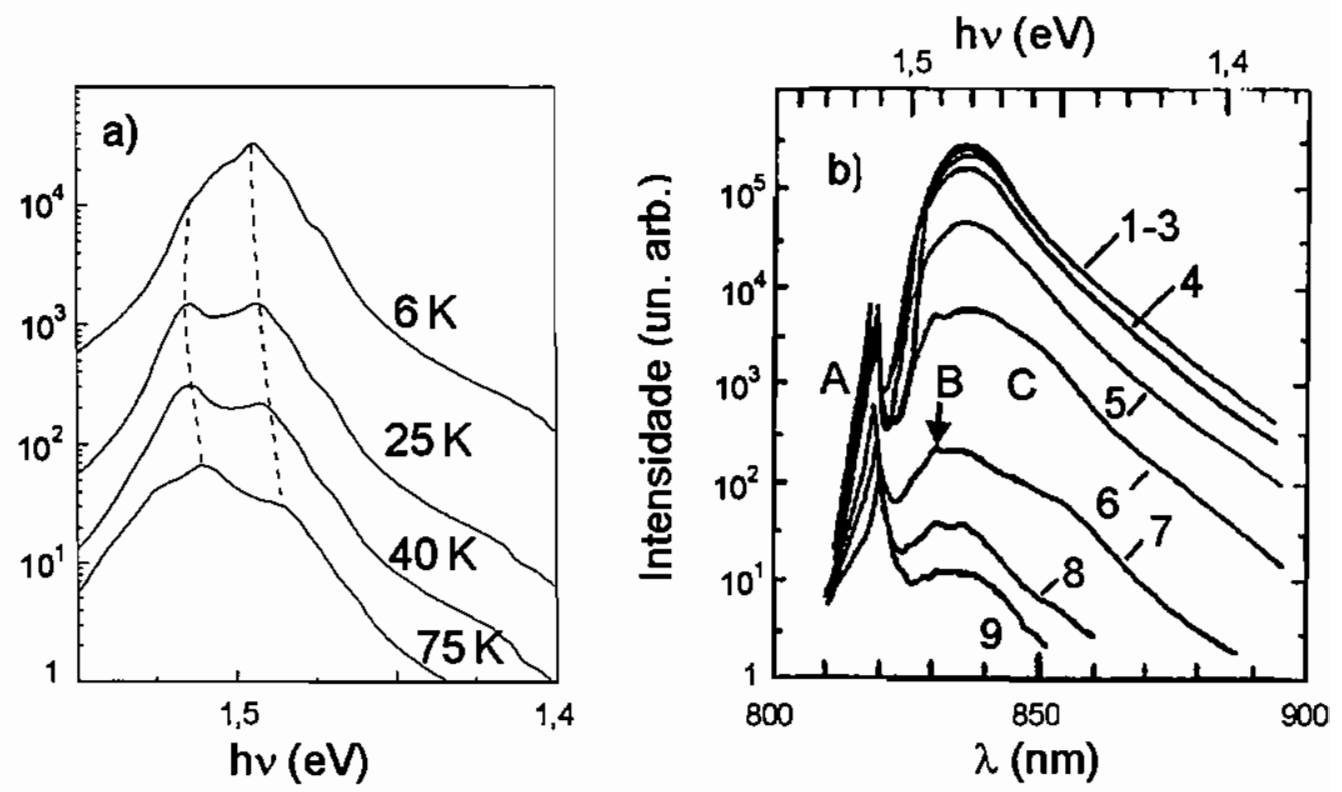

Figura 6.9: a) Espectros teóricos de fotoluminescência calculados para as temperaturas de $6 \mathrm{~K}, 25 \mathrm{~K}, 40 \mathrm{~K}$ e $75 \mathrm{~K}$, para uma super-rede com período de $2000 \AA$, com concentração $N_{A}=8,0 \times 10^{12} \mathrm{~cm}^{-2}$, largura de linha $5 \mathrm{meV}$ e fundo de portadores minoritários $N_{s}=2 \times 10^{11} \mathrm{~cm}^{-2}$. b) Espectros experimentais extraídos da ref. [27] para um poço isolado com $N_{A}=3,9 \times 10^{12} \mathrm{~cm}^{-2}$ às seguintes temperaturas: 1) $5 \mathrm{~K}$, 2) $\left.10 \mathrm{~K}, 3\right) 15 \mathrm{~K}$, 4) $20 \mathrm{~K}$, 5) $25 \mathrm{~K}, 6) 30 \mathrm{~K}, 7) 40 \mathrm{~K}, 8) 50 \mathrm{~K} \mathrm{e} \mathrm{9)} 60 \mathrm{~K}$.

fotogerados $p_{\text {foto }}=2 \times 10^{11} \mathrm{~cm}^{-2}$, um limite superior, considerando que todas as impurezas adicionadas ao sistema fossem ionizadas. Nesta figura, podemos ver claramente o poço para elétrons criado pela carga fotoinduzida. O espectro de luminescência para este sistema é apresentado na fig. 6.8 b, para as mesmas temperaturas que na fig. 6.8 a. Como esperado o aumento de temperatura, causa um decréscimo na intensidade da emissão. As intensidades dos espectros para o sistema com portadores fotoinduzidos são cinco vezes maiores que as para o sistema sem esses portadores.

Retornando aos resultados apresentados na fig. 6.9, mostramos uma 
comparação entre um espectro calculado de luminescência (à esquerda) e espectros experimentais extraídos da ref. [27] (à direita). Os espectros teóricos foram obtidos para uma super-rede com período $\mathrm{d}=2000 \AA$, $\Delta z=75 \AA$ e concentração de $N_{A}=8,0 \times 10^{12} \mathrm{~cm}^{-2}$. Os únicos dados dos espectros experimentais se referem à concentração de impurezas na camada $\delta, N_{A}=3,9 \times 10^{12} \mathrm{~cm}^{-2}$. Se os picos excitônicos fossem acrescentados aos espectros calculados da fig. 6.9 a os mesmos seriam muito similares aos da fig. 6.9 b. Esta é uma indicação clara de que o poço fotoinduzido para elétrons é responsável pelo forte decréscimo da intensidade do pico devido ao poço $\delta$-doping tipo p com o aumento da temperatura.

Analisando os resultados obtidos, podemos tirar algumas conclusões a respeito de espectros de luminescência de estruturas $\delta$-doping tipo $\mathrm{p}$ em GaAs:

1. Devido ao curto alcance das interações do potencial das impurezas as transições ópticas são principalmente indiretas. Transições diretas resultam em espectros com um maior número de estruturas e com estruturas muito mais estreitas que os espectros obtidos experimentalmente. Já no caso de transições indiretas, os espectros apresentam picos bem resolvidos, comparáveis com os obtidos experimentalmente. Em muitos casos os picos não podem ser atribuídos a transições para uma única minibanda de buracos, mas são resultado de transições para duas ou mais minibandas.

2. Os espectros teóricos ou experimentais dependem não apenas dos estados confinados de buracos mas também dos estados extendidos de elétrons. Comparando resultados experimentais e teóricos deve-se tomar cuidado pois elétrons e buracos estão sujeitos ao mesmo potencial, como acontece em uma situação real. Este ponto deve ser muito mais crítico para os elétrons que para os buracos. Em uma super-rede de período $500 \AA$, por exemplo, os poços são isolados no que se refere aos buracos. Quanto aos elétrons porém, é necessário uma super-rede de período de aprox. $2000 \AA$ para que a barreira que a camada $\delta$ representa para os buracos se assemelhe a uma barreira isolada. 
3. Se o confinamento de elétrons não for incluído, a teoria prevê, para as estruturas do espectro relacionadas ao poço, um aumento do sinal de PL em função do aumento da temperatura. A discrepância entre esta previsão e os resultados experimentais que mostram uma redução do sinal referente ao poço $\delta$-doping, é resolvida com a inclusão do confinamento de elétrons fotocriados no modelo. 


\section{Capítulo 7}

\section{Conclusão}

\subsection{O trabalho desenvolvido}

Neste trabalho desenvolvemos um método para o estudo teórico de heteroestruturas $\delta$-doping tipo p baseado na aproximação da massa efetiva e no método autoconsistente ${ }^{[56,77,79]}$.

Apesar de sua primeira formulação ter aparecido na década de 50 , a aproximação da massa efetiva ainda se mostra eficaz para resolver problemas, se não impossíveis, muito difíceis de se calcular com métodos $a b$ initio. A utilização de ondas planas em nosso cálculo simplificou a descrição dos potenciais, facilitando o trabalho. Esta era uma escolha anteriormente difícil, pois aumentava muito as necessidades computacionais.

Essas escolhas tornaram possível o cálculo eficaz da estrutura de bandas de poços $\delta$-doping tipo p em GaAs, nossa meta inicial. Junto ao cálculo da estrutura de bandas, obtivemos o potencial a que os portadores estão submetidos, suas distribuições de carga, suas funções de onda e o nível de Fermi à $\mathrm{T}=0 \mathrm{~K}$.

Para se chegar ao resultado final uma das principais dificuldades foi 
achar uma nova formulação para o termo de troca e correlação do potencial, dentro da aproximação de densidade local que incluísse diferentes tipos de portadores. A partir da aproximação de Hedin-Lundqvist para o termo de troca e correlação par um gás de elétrons inomogêneo, foi desenvolvida uma nova aproximação para aplicação no gás de buracos que se mostrou bastante importante no decorrer do trabalho.

A necessidade de se comparar os resultados obtidos com resultados experimentais, gerou uma nova ênfase em uma segunda etapa do trabalho. Foi feito um programa de cálculo teórico de espectros de luminescência a partir dos resultados obtidos do cálculo da estrutura de bandas.

Todos os métodos e aproximações foram testados e suas limitações ponderadas.

\subsection{Principais contribuições}

O método de cálculo da estrutura de bandas por nós desenvolvido dispensa as aproximações ao Hamiltoniano $\mathbf{k} \cdot \mathbf{p}$ utilizada em trabalhos anteriores. Outra contribuição de nosso método é o tratamento adequado para o potencial de troca e correlação para um gás de buracos dentro da aproximação da densidade local ${ }^{[80]}$. Estudos anteriores não haviam contemplado adequadamente este termo, o que era um procedimento plenamente justificado em alguns casos. O tratamento apresentado abre a possibilidade de um estudo mais aprofundado de estruturas onde o potencial dos portadores é fundamental.

Foi realizado também um estudo sistemático da estrutura de bandas de poços $\delta$-doping tipo p, variando-se diversos parâmetros: concentração de portadores $\left(N_{A}\right)$, período da super-rede $(d)$ e dispersão em torno da camada de dopantes $(\Delta z)$. Com isso, uma das motivações fundamentais deste trabalho, fornecer aos pesquisadores experimentais um guia a respeito da estrutura de sub-bandas e minibandas destas estruturas, foi executada com êxito.

Este trabalho mostra ainda que é viável a simulação de espectros de 
luminescência com um bom grau de realismo. O estudo de espectros simulados com base em parâmetros extraídos dos dados nominais da amostra (dopagem nominal, período da super-rede, etc.) abre a possibilidade de discernir, em resultados experimentais, entre fenômenos causados por estruturas $\delta$-doping tipo p e fenômenos causados por outras estruturas presentes na amostra. O estudo da variação de intensidade do pico com o aumento da temperatura, feito no final do capítulo 6 , é uma primeira tentativa bastante bem sucedida neste sentido.

\subsection{Perspectivas}

Apesar da fase de desenvolvimento inicial deste trabalho ter sido superada, ainda há muito a ser extraído do método aqui desenvolvido. $O$ estudo das propriedades dos sistemas $\delta$-doping tipo p em GaAs pode ainda ser aprofundado, ampliando o universo dos valores de cada parâmetro estudados. Uma tentativa nesta direção foi recentemente iniciada por nós através do estudo sistemático da estrutura de bandas de um número significativamente maior de super-redes ${ }^{[81]}$.

Apesar de termos obtido resultados muito interessantes de fotoluminescência ${ }^{[78]}$, novos espectros de luminescência devem também ser calculados. $\mathrm{O}$ intuito de tal estudo continua sendo o de fornecer aos pesquisadores experimentais um guia a respeito do fenômeno. A esse mapeamento deve ser incorporado ainda um estudo mais aprofundado do comportamento dos portadores fotogerados.

Outros efeitos, como a presença de campo magnético, devem ser ainda incorporados ao formalismo e ao programa e estudados. Uim estudante do LNMS, treinado no programa aqui desenvolvido faz atualmente um estudo para a introdução de campo magnético nos sistemas aqui estudados.

O estudo de poços $\delta$-doping em silício é também uma possibilidade bastante concreta e interessante. A banda 'split-off' do silício é separada da banda dos buracos leves e pesados por apenas $44 \mathrm{meV}$. Dessa for- 
ma além dos estados de buracos leves e pesados, os buracos 'split-off' também podem ser ocupados. O programa desenvolvido já vem sendo utilizado por uma estudante do grupo para calcular a estrutura de bandas de poços $\delta$-doping tipo p em silício[82].

Outra possibilidade é a generalização deste método para heteroestruturas composicionais, possibilitando assim o estudo de estruturas que misturem materiais de diferentes composições com poços e barreiras de dopagem modulada. Esta generalização proporcionará um estudo ainda mais realista destes sistemas.

Também seria desejável um incremento na velocidade de cálculo e no tamanho dos sistemas. Isso tornaria possível o cálculo de estruturas mais complexas, tais como super-redes dentes de serra ou envolvendo poços de diferentes concentrações.

Finalmente salientamos a importância que terá o trabalho aqui desenvolvido para a pesquisa experimental que o grupo do LNMS vem realizando. Estão sendo crescidas no grupo super-redes e poços isolados $\delta$-doping tipo p em GaAs na direção [311]. O estudo de sistemas deste tipo exige poucas alterações no programa. Abre-se assim a perspectiva, muito interessante, de se combinar resultados obtidos através do método que desenvolvemos neste trabalho com resultados experimentais destas amostras. 


\section{Apêndice A}

\section{Programas desenvolvidos}

Neste apêndice farei uma descrição sucinta dos programas e sub-rotinas por mim desenvolvidos. Para o cálculo da estrutura de bandas descreverei o programa baseado no Hamiltoniano $8 \times 8$ de Kane. Por ser uma evolução do programa anterior (baseado no Hamiltoniano $4 \times 4$ de Luttinger-Kohn), o entendimento do método baseado no Hamiltoniano de Kane segue o mesmo esquema. A necessidade de portar os programas entre diversas plataformas diferentes nos fez adotar o FORTRAN 77, e o padrão ANSI do mesmo como linguagem de desenvolvimento. Os programas são também estruturados, facilitando o portabilidade dos algoritmos desenvolvidos para outras linguagens de desenvolvimento.

\section{A.1 Cálculo da estrutura de bandas}

Os dados de entrada do programa do cálculo da estrutura de banda são:

A concentração de portadores na amostra, composta da soma da distribuição de cargas, causada pelo plano de impurezas com a distribuição de cargas proveniente de um fundo de impurezas inerente ao sistema. 
As componentes em ondas planas dos potenciais gerados pela camada de impurezas e pelos portadores, calculados por uma rotina preliminar.

O número de ondas planas utilizados para descrever o potencial do sistema.

O comprimento da supercélula a ser calculada.

Os parâmetros de Luttinger-Kohn ou de Kane, o 'gap', o tamanho da supercélula ' $\mathrm{D}$ ', a constante dielétrica e o parâmetro de rede do sistema.

O número de iterações e o número de pontos em cada direção da rede a ser construída.

Depois de lidos os dados, o programa do cálculo da estrutura de bandas executa o processo autoconsistente e por fim, escreve os dados resultantes numa forma conveniente. Por serem duas etapas distintas, vamos chamá-las de autoconsistência e escrita.

A autoconsistência se faz em três passos independentes um do outro: a determinação dos auto-estados e seus autovalores, o cálculo do nível de Fermi a $\mathrm{T}=0 \mathrm{~K}$ e a determinação do novo potencial através da integral dupla da equação de Poisson. Por este motivo, a autoconsistência é dividida em três sub-rotinas independentes, uma para cada procedimento: DIAGO, FERMI e POISSON.

Na sub-rotina DIAGO uma malha é construída, definido-se cada ponto do espaço recíproco a ser utilizado no cálculo. Para cada um destes pontos escreve-se o Hamiltoniano de Kane e somam-se os potenciais coulombiano e de troca e correlação. Depois de obtida a matriz do Hamiltoniano completo diagonaliza-se a mesma e armazenam-se os dados dos trinta primeiros estados das bandas de valência e condução em cada ponto em vetores específicos: um para os autovalores e um para os autovetores dos elétrons na banda de condução, um para os autovalores e um último para os autovetores dos buracos na banda de valência. 
A sub-rotina DIAGO vale-se de outras sub-rotinas para executar partes específicas do processo. A sub-rotina HAMILTON escreve o termo de energia cinética do Hamiltoniano e soma ao mesmo o termo referente ao potencial coulombiano. A sub-rotina HAMXC soma ao Hamiltoniano o termo de troca e correlação, determinando assim o potencial total a ser diagonalizado pela rotina $\mathrm{CH}$ (extraída do pacote EISPACK).

Para a determinação do termo de troca e correlação é necessário que a distribuição de cargas dos portadores seja escrita no espaço direto, sua raiz cúbica seja extraída e esse valor multiplicado por uma constante, antes de aplicar novamente a transformada de Fourier, determinando os coeficientes do termo no espaço recíproco. Para escrever a distribuição de cargas no espaço direto é utilizada a sub-rotina CARGA e para os outros passos do processo a sub-rotina TROCA.

De posse dos autovalores dos portadores majoritários, pode-se calcular o nível de Fermi a $\mathrm{T}=0 \mathrm{~K}$, para a amostra considerada. A sub-rotina FERMI executa este cálculo escolhendo um nível de Fermi tentativo e somando a carga de todos os estados cuja energia seja menor que a energia de Fermi. Caso a carga obtida seja igual à dos portadores, lida com os dados de entrada, o processo se interrompe. Caso contrário, uma nova energia é escolhida, somam-se novamente todos os estados com energia inferior à tentativa e compara-se novamente o valor calculado com o de entrada. Repete-se esse processo até que os dois valores coincidam. Depois de determinado o nível de Fermi comparam-se as energias de todos os autovalores com o mesmo e num novo vetor armazena-se a ocupação do estado.

A sub-rotina POISSON determina os coeficientes do novo potencial dos portadores. Para tanto ela calcula o coeficiente $P$ através da eq. (3.26), divide o mesmo pelo quadrado da diferença do número de ondas planas relativos aos índices das equações e multiplica pelas constantes necessárias, calculando o segundo termo do lado esquerdo da eq. (3.27).

De posse dos novos valores para o potencial dos portadores, recomeçase todo o processo de cálculo a partir da sub-rotina DIAGO. Quando o número de vezes que esse processo foi refeito igualar com o número de iterações lido com os dados de entrada passa-se ao procedimento de 
escrita dos dados numa forma conveniente.

A sub-rotina POTDIR escreve o potencial no espaço direto no arquivo 'pot.dat', de maneira a poder-se desenhá-lo diretamente. As sub-rotinas WAVE e ESCWV, escrevem o quadrado das funções dos portadores majoritários e minoritários no ponto $\Gamma$ nos arquivos 'hwvfsqrg.dat' e 'ewvfsqrg.dat' e no ponto $Z$ nos arquivos 'hwvfsqrz.dat' e 'ewvfsqrz.dat'. As sub-rotinas VALX, VALZ e VALY escrevem as estruturas das bandas de valência e condução nas direções $\Gamma-\Delta, \Gamma-Z$ e $\Gamma-\Delta^{\prime}$ respectivamente, nos arquivos 'hbandst.dat' e 'ebandst.dat'.

\section{A.2 Cálculo do espectro de luminescência}

O cálculo do espectro de luminescência é feito por um programa que incorpora o anterior e após o termino do processo de cálculo da estrutura de bandas executa uma série de sub-rotinas escritas para este fim.

Além dos dados de entrada do programa anterior este precisa de dados adicionais:

As temperaturas dos espectros a serem calculados.

As larguras de linha das transições, introduzidas ad hoc.

Após o processo de cálculo da estrutura de bandas executa-se a subrotina OCUPACAO, que de posse dos valores de ocupação dos estados a $\mathrm{T}=0 \mathrm{~K}$, recalcula os valores a temperaturas finitas. Para tal vale-se da função de distribuição de Fermi para os portadores majoritários e da função de distribuição de Boltzmann para os portadores minoritários.

Em seguida são calculados os espectros. Para os espectros incluindo apenas transições diretas é utilizada a sub-rotina ESPECDIR e para os espectros incluindo também transiçōes indiretas é utilizada a subrotina ESPECTRO. Ambas as rotinas calculam as forças de oscilador entre estados eletrônicos e de buracos segundo a expressão da eq. (4.17) 
e multiplicam as forças de oscilador pelas ocupações dos estados, obtendo a intensidade da transição. Em seguida toma-se uma função Lorentziana normalizada e multiplica-se a mesma por essa intensidade. O espectro é o resultado da soma das Lorentzianas obtidas.

Por último, pode-se calcular somente a força de oscilador. A sub-rotina INTEGRAL calcula a força de oscilador da transição entre um estado da banda de valência e estados na banda de condução, mapeando-a através de transições diretas entre estados da banda de valência e da banda de condução, no ponto $\Gamma$.

\section{A.3 Determinação das componentes do potencial}

Entre os dados de entrada dos programas de cálculo da estrutura de bandas e da luminescência figuram as componentes em ondas planas do potencial das impurezas e de um potencial tentativo inicial para os portadores. O programa POT constrói os potenciais no espaço direto e calcula os coeficientes de Fourier dos mesmos, escrevendo-os na forma necessária aos programas seguintes no arquivo chamado 'vfinal.dat'.

O potencial tentativo dos portadores resume-se ao potencial de uma distribuição Gaussiana de cargas com carga de sinal contrário à das impurezas e largura $\sigma$ maior que a Gaussiana da distribuição das impurezas. O valor usual para essa grandeza é o dobro da distribuição das impurezas. Dependendo do sistema a ser calculado é necessário um ajuste desse valor. 


\section{Bibliografia}

[1.] E. F. Schubert, em Epitaxial Microstructures, editado por Arthur C. Gossard, Semicondutors and Semimetals Vol. 40 (Academic, New York, 1994), p. 1.

[2] K. Ploog, J. Cryst. Growth 81, 304 (1987).

[3] E. F. Schubert, J. Vac. Sci. Technol. A 8, 2980 (1990).

[4] H. P. Zeindl, T. Wegehaupt, I. Eisele, H. Oppolzer, H. Reisinger, G. Tempel e F. Koch, Appl. Phys. Lett. 50, 1164 (1987).

[5] N. L. Mattey, M. G. Dowsett, E. H. C. Parker, T. E. Whall, S. Taylor, e J. F. Zhang, Appl. Phys. Lett. 57, 1648 (1990).

[6] A. Zrenner, F. Koch e K. Ploog, Surf. Sci. 196, 671 (1988).

[7] M. Santos, T. Sajoto, A. Zrenner e M. Shayegan, Appl. Phys. Lett. 53, 2504 (1988).

[8] M. H. Degani, J. Appl. Phys. 70, 4362 (1991).

[9] S. M. Shibli, L. M. R. Scolfaro, J. R. Leite, C. A. C. Mendonça, F. Plentz e E. A. Meneses, Appl. Phys. Lett. 60, 2895 (1992).

[10] C. A. C. Mendonça, F. Plentz, J. B. B. Oliveira, E. A. Meneses, L. M. R. Scolfaro, D. Beliaev, S. M. Shibli e J. R. Leite, Phys. Rev. B 48, 12316 (1993).

[1.1] L. Chico, F. García-Moliner e V. R. Velasco, Phys. Rev. B 48, 11427 (1993). 
[12] H. Jorke, H. Kibbel, F. Schäfller, A. Casel, H.-J. Herzog e E. Kasper, Appl. Phys. Lett. 54, 819 (1989).

[13] W. Kiunke, E. Hammerl, I. Eisele, D. Schulze e G. Gobsch, J. Appl. Phys. 72, 3602 (1992).

[14] H.-J. Gossmann e F. C. Unterwald, Phys. Rev. B 47, 12618 (1993).

[15] C. A. C. Mendonça, L. M. R. Scolfaro, J. B. Oliveira, F. Plentz, F. Mikovic, J. R. Leite e E. A. Meneses, Superlatt. Microstruct. 12, 257 (1992).

[16] W.-X. Ni, G. V. Hansson, J.-E. Sundgren, L. Hultman, L. R. Wallenberg, J.-Y. Yao, L. C. Markert e J. E. Greene, Phys. Rev. B 46, 7551 (1992).

[1.7] R. Enderlein, L. M. R. Scolfaro, J. M. V. Martins, J. R. Leite, F. Plentz, S. M. Shibli e E. A. Meneses, Superlatt. Microstruct. 12, 175 (1992).

[18] M. E. Lazzouni e L. J. Sham, Phys. Rev. B 48, 8948 (1993).

[19] L. M. R. Scolfaro, A. T. Lino, E. K. Takahashi e J. R. Leite, Int. J. Quantum Chem. S28, 667 (1994).

[20] M. H. Degani, Phys. Rev. B 44, 5580 (1991).

[21] L. M. R. Scolfaro, D. Beliaev, R. Enderlein e J. R. Leite, Phys. Rev. B 50, 8699 (1994).

[22] R. Enderlein, L. M. R.Scolfaro, e J. R. Leite, Phys. Rev. B 50, 18312 (1994).

[23] S. J. Pearton, F. Ren, C. R. Abernathy, W. S. Hobson, S. N. J. Chu, e J. Kovalchik, Appl. Phys. Lett. 55, 1342 (1989).

[24] F. Schubert, J. M. Kuo, R. F. Kopf, H. S. Luftmann, C. L. Hopkins, e N. J. Sauer, J. Appl. Phys. 67, 1969 (1990). 
[25] O. Ourmazd, J. Cunningham, W. Jan, J. A. Rentschler, e F. Schröter, Appl. Phys. Lett. 56, 854 (1990).

[26] J. Wagner, A. Ruiz e K. Ploog, Phys. Rev. B 43, 12134 (1991).

[27] A. M. Gilinsky, K. S. Zhuravlev, D. I. Lubyshev, V. P. Migal,V. V. Preobrashenskii e B. R. Semiagin, Superlatt. Microstruct. 10, 399 (1991).

[28] D. Richards, J. Wagner, H. Schneider, G. Hensdorfer e M. Maier, Phys. Rev. B 47, 9629 (1993).

[29] M. El Allali, C. B. Sorensen e E. Veje, J. Phys. (France) Colloq. IV C5, 299 (1993).

[30] V. Ya. Aleshkin, A. V. Anshon, L. M. Batukova, E. V. Demidov, E. R. Demidova, B. N. Zvonkov, I. A. Karpovich e I. G. Malkina, Soviet Phys. Semic. 26, 1038 (1992).

[31] Dan Ritter, R. A. Hamm, M. B. Panish e M. Geva, Appl. Phys. Lett. 63, 1543 (1993).

[32] M. Ilegems, J. Appl. Phys. 48, 1278 (1977).

[33] P. M. Koenraad, M. B. Johnson, H. W. M. Salemink, W. C. van der Vleuten e J. H. Wolter, Mater. Sci. Eng. B 35, 485 (1995).

[34] L. Hedin e J. Lundqvist, J. Phys. C 4, 2064 (1971).

[35] F. A. Reboredo e C. R. Proetto, Phys. Rev. B 47, 4655 (1993).

[36] J. M. Luttinger e W. Kohn, Phys. Rev. 97, 869 (1955).

[37] U. Ekenberg e M. Altarelli, Phys. Rev. B 30, 3569 (1984).

[38] D. A. Broido e L. J. Sham, Phys. Rev. B 31, 888 (1985).

[39] E. Bangert e G. Landwehr, Superlatt. Microstruct. 1, 363 (1985).

[40] T. Ando, J. Phys. Soc. Jpn. 54, 1528 (1985). 
[41] M. Altarelli, U. Ekenberg e A. Fasolino, Phys. Rev. B 32, 5138 (1985).

[42] G. Platero e M. Altarelli, Phys. Rev. B 39, 3758 (1989).

[43] E. Bangert, K. von Klitzing e G. Landwehr, em Proceedings of the 12th International Conference on the Physics of Semiconductors, editado por M. H. Pilkhun (Teubner Verlag, Stuttgart, 1974), p. 714.

[44] E. Bangert e G. Landwehr, Surf. Sci. 58, 138 (1976).

[45] F. J. Ohkawa e Y. Uemura, Suppl. Progr. Theor. Phys. 57, 164 (1975).

[46] S. S. Nedorezov, Soviet Phys. Solid St. 12, 1814 (1971).

[47] F. J. Ohkawa, J. Phys. Soc. Jpn. 41, 122 (1976).

[48] T. Ando, Phys. Rev. B 13, 3468 (1976).

[49] T. Ando, J. Phys. Soc. Jpn. 51, 3893 (1982).

[50] F. Stern e S. Das Sarma, Phys. Rev. B 30, 840 (1984).

[51] I. V. Kukushkin, K. von Klitzing, K. Ploog, V. E. Kirpchev, e Nb. N. Shegel, Phys. Rev. B 40, 4179 (1989).

[52] I. V. Kukushkin, K. von Klitzing, K. Ploog e V. E. Timofeev, Phys. Rev. B 40, 7788 (1989).

[53] I. A. Buyanova, W. M. Chen, A. Henry, W.-X. Ni, G. V. Hanson e B. Monemar, Phys. Rev. B 53, 9587 (1996).

[54] G. Bastard, Wave mechanics applied to semiconductor heterostructures (Les editions de physique, Paris, 1988).

[55] J. R. Chelikowsky e M. L. Cohen, Bandstructure and optical properties of semiconductors, Springer 1987

[56] R. Enderlein, G. M. Sipahi, L. M. R. Scolfaro e J. R. Leite, submetido ao Phys Rev. Lett.(1997) 
[57] E. O. Kane, em Physics of III-V Compounds, editado por R. K. Willardson e A. C. Beer, Semicondutors and Semimetals Vol. 1 (Academic, New York, 1966), p. 75.

[58] Abraham Moysés Cohen e Gilmar Eugenio Marques, Phys. Rev. B 41, 10608 (1990).

[59] Abraham Moysés Cohen, Tese de Doutoramento, Instituto de Física e Química de São Carlos, Universidade de São Paulo, 1990.

[60] D. M. Wood e A. Zunger, Phys. Rev. B 53, 7949 (1996).

[61] D. Gershoni, C. H. Henry e G. A. Baraff, IEEE J. Quantum Electron. 29, 2433 (1993).

[62] L. R. Ram-Mohan, K. H. Yoo e R. L. Aggrawall, Phys. Rev. B 38, 6151 (1986).

[63] G. Harbeke, O. Madelung e U. Rössler, in Physics of Group IV Elements and III-V Compounds, editado por O. Madelung, LandoldtBörnstein, New Series, Group III, Vol. 17, Pt a (Springer-Verlag, Berlin, 1982).

[64] M. Combescot e P. Nozières, J. Phys. C 5, 2369 (1972).

[65] R. O. Jones e O. Gunnarson, Rev. Mod. Phys. 61, 689 (1989).

[66] Y. P. Varshni, Physica 34, 149 (1967).

[67] R. C. Miller, D. A. Kleinman e A. C. Gossard, Phys. Rev. B 29, 7085 (1984).

[68] L. Ioriatti, Phys. Rev. B 43, 14742 (1991).

[69] L. M. R. Scolfaro, R. P. Camata, J. M. V. Martins e J. R. Leite, Superlatt. Microstruct. 12, 203 (1992).

[70] D. Beliaev, L. M. R. Scolfaro e J. R. Leite, Semicond. Sci. Technol. 8, 1479 (1993).

[71] D. Beliaev, L. M. R. Scolfaro e J. R. Leite, Bras. J. Phys. 24, 270 (1993). 
[72] J. R. Leite, S. C. P. Rodrigues, L. M. R. Scolfaro, R. Enderlein, D. Beliaev e A. A. Quivy, Mater. Sci. Eng. B 35, 250 (1995).

[73] A. Marti Ceschin, A. A. Quivy, J. A. N. T. Soares, R. Enderlein, A. Tabata, L. M. R. Scolfaro, E. C. F. Silva, J. R. Leite, J. B. B. Oliveira e E. A. Menezes, Superlatt. Microstruct. 15, 333 (1994).

[74] A. Tabata, A. M. Ceschin, A. A. Quivy, A. Levine, J. R. Leite, R. Enderlein, J. B. B. Oliveira, E. Lauretto e J. L. Gonçalves, Mater. Sci. Eng. B 35, 401 (1995).

[75] Yu. A. Pusep, M. T. O. Silva, J. C. Galzerani, S. W. da Silva, L. M. R. Scolfaro, R. Enderlein e J. R. Leite, Phys. Rev. B 54, 13927 (1996).

[76] S. C. P. Rodrigues e L. M. R. Scolfaro, comunicação pessoal.

[77] G. M. Sipahi, R. Enderlein, L. M. R. Scolfaro e J. R. Leite, em Proceedings of the 22nd International Conference on the Physics of Semiconductors, editado por David J. Lockwood (World Scientific, Singapore, 1995), p. $687 .$.

[78] R. Enderlein, G. M. Sipahi, L. M. R. Scolfaro, J. R. Leite, e I. F. L. Diaz, Mater. Sci. Eng. B 35, 396 (1995).

[79] G. M. Sipahi, R. Enderlein, L. M. R. Scolfaro e J. R. Leite, Phys. Rev. B 53, 9930 (1996).

[80] G. M. Sipahi, R. Enderlein, L. M. R. Scolfaro e J. R. Leite, em Proceedings of the 7th International Conference on Shalow Level Centers on Semiconductors, editado por C. A. J. Ammerlaan e B. Pajot (World Scientific, Singapore, 1996), p. 209..

[81] L. E. Ramos, G. M. Sipahi, L. M. R. Scolfaro, R. Enderlein e J. R. Leite, submetido ao Superlatt. Microstruct.(1997).

[82] A. L. Rosa, L. M. R. Scolfaro, G. M. Sipahi, R. Enderlein e J. R. Leite, Mater. Sci. Eng. B (1997), aceito para publicaçāo. 